\title{
A systematic method for constructing time discretizations of integrable lattice systems: local equations of motion
}

\author{
Takayuki TsuchIDA* \\ Okayama Institute for Quantum Physics, \\ Kyoyama 1-9-1, Okayama 700-0015, Japan
}

October 26, 2018

\begin{abstract}
We propose a new method for discretizing the time variable in integrable lattice systems while maintaining the locality of the equations of motion. The method is based on the zero-curvature (Lax pair) representation and the lowest-order "conservation laws". In contrast to the pioneering work of Ablowitz and Ladik, our method allows the auxiliary dependent variables appearing in the stage of time discretization to be expressed locally in terms of the original dependent variables. The time-discretized lattice systems have the same set of conserved quantities and the same structures of the solutions as the continuous-time lattice systems; only the time evolution of the parameters in the solutions that correspond to the angle variables is discretized. The effectiveness of our method is illustrated using examples such as the Toda lattice, the Volterra lattice, the modified Volterra lattice, the Ablowitz-Ladik lattice (an integrable semi-discrete nonlinear Schrödinger system), and the lattice Heisenberg ferromagnet model. For the Volterra lattice and modified Volterra lattice, we also present their ultradiscrete analogues.
\end{abstract}

Keywords: integrable lattices, time discretization, Lax pair, Toda lattice, Volterra lattice, Ablowitz-Ladik lattice, local fully discrete NLS, ultradiscretization, ultradiscrete (modified) KdV hierarchy

PACS numbers: 02.30.Ik, 02.70.Bf, 05.45.Yv, 45.05.+x

${ }^{*}$ E-mail: surname at ms.u-tokyo.ac.jp 


\section{Contents}

1 Introduction

2 Method for time discretization $\quad 5$

2.1 Lax pair and a conservation law in the continuous-time case . 6

2.2 Lax pair in the discrete-time case and auxiliary variables . . . 7

2.3 Fundamental "conservation laws" in the discrete-time case . . 9

2.4 Algebraic system for the auxiliary variables and local equations of motion . . . . . . . . . . . . . . 10

2.5 Remarks on nonautonomous extensions . . . . . . . . . . 11

3 Examples $\quad \mathbf{1 4}$

3.1 The Toda lattice in Flaschka-Manakov coordinates . . . . . . 14

3.2 The Ablowitz-Ladik lattice . . . . . . . . . . . . . . . 20

3.3 The Volterra lattice . . . . . . . . . . . . . . . . . 36

3.4 The modified Volterra lattice . . . . . . . . . . . . . . . . 45

3.5 The lattice Heisenberg ferromagnet model . . . . . . . . . . . 51

4 Concluding remarks

$\begin{array}{ll}\text { References } & 58\end{array}$ 


\section{Introduction}

The quest for a finite-difference analogue of a given differential equation can be justified for several sound reasons. A suitable discretization can reproduce most of the important properties of the differential equation in the smallvalue range of the difference interval and can be considered a "generalization" of the original continuous equation. Such a discretization facilitates a better and more intuitive understanding of the differential equation without using a limiting procedure, which is needed to define differentiation, and is ideal for performing numerical experiments.

The suitable discretization of a completely integrable system is usually required to retain the integrability; if this is satisfied, it is called an integrable discretization. An integrable system often admits more than one integrable discretization; in such a case, we can consider the properties of each integrable discretization other than integrability and discuss which one is the most favorable for our purpose. The problem of integrable discretization has been sporadically studied since the mid-1970s, i.e., the dawn of the modern theory of integrable systems. For more than thirty years, various techniques have been developed to obtain integrable discretizations of continuous systems. Readers interested in the history of integrable discretizations are referred to the preface of Suris's book [1].

Partial differential equations (PDEs) involve more than one independent variable. The discretization of an integrable nonlinear PDE is generally performed in two steps; in the first step, we discretize the spatial variable(s) and in the second step, the time variable is discretized. Of course, for some PDEs such as the sine-Gordon equation $u_{x t}=\sin u$, the roles of the space and time variables can be swapped and it is not meaningful to discuss the order of discretization. However, for an integrable nonlinear PDE wherein the roles of individual variables are essentially different and not interchangeable, the order in which the independent variables may be discretized appears to be unique. Thus, we can consider that the time variable is always discretized last, after the spatial variable(s) have been discretized.

In this paper we focus on the problem of the integrable full discretization of differential-difference equations in $1+1$ dimensions. The continuous independent variable to be discretized is regarded as time, as noted above. Most of the differential-difference equations considered reduce to integrable PDEs in a proper continuous limit; however, this is not necessary and we can also start with integrable lattice systems that have no continuous counterpart. The problem of time discretization is, by its nature, distinct from the problem of space discretization. In fact, the former problem has its own peculiarities and difficulties that the latter does not have. This point was 
uncovered by Ablowitz and Ladik [2, 3] in their attempt to fully discretize the space-discretized nonlinear Schrödinger (semi-discrete NLS) equation [4]. It turned out that unexpected nonlocality emerges in the stage of time discretization; the fully discrete NLS equation involves infinite sums and/or infinite products (in the case of an infinite chain) with respect to the discrete spatial variable and is thus a global-in-space scheme. The fully discrete NLS equation can superficially be written in a local form using additional dependent variables called auxiliary variables, but it does not provide any essential resolution of the nonlocality problem. The subsequent paper by Taha and Ablowitz [5] reinforces the impression that the appearance of global terms is a general feature of the problem of time discretization for integrable lattice systems.

The pioneering work of Ablowitz and coworkers [2, 3, 5] is based on the zero-curvature (Lax pair) representation; the guiding principle is that the time discretization does not change the spatial part of the Lax pair for an integrable lattice system. This automatically guarantees the major advantages of the full discretization; that is, the time-discretized lattice systems have the same integrals of motion and the same structures of the solutions as the original continuous-time lattice systems. In more modern terms, each of their time discretizations belongs to the same integrable hierarchy as the underlying continuous-time system [6,7]. Despite the elegance of this result, the appearance of infinite sums/products is a shortcoming not acceptable to everybody, and new ideas are needed to remove the nonlocality. In this regard, Suris recently introduced the notion of localizing changes of variables, applied it to a large number of integrable lattice systems, and obtained their time discretizations written in local equations of motion [1]. Note that some of his discretizations coincide with earlier results obtained using $\mathrm{Hi}$ rota's bilinear method [8], as described in the bibliographical remarks in his book [1]. Suris's idea was to find, by guessing, a change of variables such that the equations of motion as well as the auxiliary variables can be expressed locally in terms of the new dependent variables; the change of variables involves the step size of time as a parameter and is considered as a discrete Miura transformation giving a one-parameter deformation of the original lattice hierarchy. Thus, the integrals of motion and the solution formulas for the time-discretized lattice system are deformed accordingly. Moreover, although Suris's approach has successfully provided many interesting examples, its applicability is rather limited. In particular, it is not applicable to the time discretization of NLS-type lattices, such as the semi-discrete NLS equation (also called the Ablowitz-Ladik lattice) [4], wherein the two dependent variables can be related by a complex conjugacy reduction.

The main objective of this paper is to propose a systematic method for 
constructing time discretizations of integrable lattice systems written as local equations of motion. In contrast to the other known methods, our method generally requires no ad hoc treatment on a case-by-case basis and appears to have no serious limitations in its applicability; it can be applied to possibly all lattice systems in $1+1$ dimensions possessing a Lax pair representation. In particular, it can be used to obtain local full discretizations of NLS-type lattices, including the Ablowitz-Ladik lattice [4]. Actually, our method can be considered as a completed version of the Ablowitz-Ladik approach [2, 3]; it both refines and extends their work in an essential way. A decisive breakthrough has been made by considering the lowest-order "conservation laws", derived from the zero-curvature condition written in matrix form. In the process, a critical role is played by an arbitrary parameter in the Lax pair, called the spectral parameter. The requirement that all the fluxes corresponding to the same conserved density have to essentially coincide results in an "ultralocal" algebraic system for the auxiliary variables; the simpler case where the conserved density is trivially a constant can be treated in a similar manner. Thus, by solving this algebraic system, we can restore the locality of the equations; that is, the global terms appearing in the stage of time discretization can be replaced by local expressions in terms of the original dependent variables.

This paper is organized as follows. In section 2, we describe the general method for discretizing the time variable. In section 3 , we construct time discretizations of the Toda lattice in Flaschka-Manakov coordinates [9 11], the Ablowitz-Ladik lattice, the Volterra lattice, the modified Volterra lattice, and the lattice Heisenberg ferromagnet model. As a spin-off, we obtain ultradiscrete analogues [12,13] of the Volterra lattice and modified Volterra lattice. In addition, we uncover unexpected relationships with the work of Nijhoff, Quispel, Capel et al. [14-17]. Section 4 is devoted to concluding remarks.

\section{Method for time discretization}

In this section, we discuss the problem of time discretization for a given integrable lattice system. We start with a Lax pair formulation in the continuoustime case and then proceed to discretize the time variable. A set of auxiliary variables is introduced to express the time-discretized lattice as a closed system of equations. Using the fundamental "conservation laws" derived from the Lax pair, we can obtain local expressions for the auxiliary variables in terms of the original variables. 


\subsection{Lax pair and a conservation law in the continuous- time case}

The Lax pair formulation in a semi-discrete space-time comprises a pair of linear equations,

$$
\Psi_{n+1}=L_{n}(\lambda) \Psi_{n}, \quad \Psi_{n, t}=M_{n}(\lambda) \Psi_{n}
$$

Here, $\Psi_{n}$ is a column-vector function and $n$ is the discrete spatial variable. The subscript $t$ denotes differentiation with respect to the continuous time variable $t$. The square matrices $L_{n}$ and $M_{n}$ depend on the spectral parameter $\lambda$, which is an arbitrary constant independent of $n$ and $t$. The compatibility condition of the overdetermined system (2.1) is given by [2, 4, 6, 7, 18, 19]

$$
L_{n, t}+L_{n} M_{n}-M_{n+1} L_{n}=O
$$

which is (a semi-discrete version of) the zero-curvature condition. The symbol $O$ is used to stress that this is a matrix equation. If we specify the $\lambda$-dependent matrices $L_{n}$ and $M_{n}$ appropriately, (2.2) results in a closed differential-difference system for some $\lambda$-independent quantities in $L_{n}$ and $M_{n}$. In such a case, the pair of matrices $L_{n}$ and $M_{n}$ is called a Lax pair. The matrix $L_{n}$ is usually ultralocal in the dependent variables; that is, if $L_{n}$ involves some variable, say $u_{n}$, then it does not involve shifted variables such as $u_{n \pm 1}$ and $u_{n \pm 2}$. In addition, the determinant of $L_{n}$ is required to be nonzero for generic $\lambda$ so that the spectral problem is well-posed on the entire infinite chain.

The zero-curvature condition (2.2) generates a conservation law of the following form:

$$
\frac{\partial}{\partial t} \log \left(\operatorname{det} L_{n}\right)=\boldsymbol{\Delta}_{n}^{+}\left(\operatorname{tr} M_{n}\right)
$$

Here, $\boldsymbol{\Delta}_{n}^{+}$is the forward difference operator in the spatial direction, i.e.,

$$
\boldsymbol{\Delta}_{n}^{+} f_{n}:=f_{n+1}-f_{n} .
$$

For a proper integrable lattice system, $\operatorname{det} L_{n}(\lambda)$ has to be either a timeindependent function of $\lambda$ or the exponential of a $\lambda$-independent conserved density multiplied by an overall $t$-independent factor. Indeed, if this was not satisfied, e.g., $\operatorname{det} L_{n}(\lambda)=1+\lambda u_{n}$, then the expansion of $\log \operatorname{det} L_{n}(\lambda)$ with respect to $\lambda$ would yield an infinite number of (almost) ultralocal conserved densities, say $u_{n}, u_{n}^{2}, \ldots$, and thus the lattice system would be trivial in some sense.

Note that the zero-curvature condition (2.2) has the following invariance properties: 
(a) $L_{n} \rightarrow f(\lambda) L_{n}$, where $f(\lambda)$ is a $t$-independent scalar function,

(b) $M_{n} \rightarrow M_{n}+g(\lambda) I$, where $g(\lambda)$ is an $n$-independent scalar function and $I$ is the identity matrix,

(c) $L_{n} \rightarrow \exp \left(\alpha \rho_{n}\right) L_{n}$ and $M_{n} \rightarrow M_{n}+\alpha j_{n} I$, where $\alpha$ is a parameter, $\rho_{n}$ is a conserved density and $j_{n}$ is the corresponding flux (up to a sign), namely, $\partial_{t} \rho_{n}=\boldsymbol{\Delta}_{n}^{+} j_{n}$.

In particular, using (2.3) and the above properties, we can convert the Lax pair to a normalized form, i.e., $\operatorname{det} L_{n}=1$ and $\operatorname{tr} M_{n}=0$.

\subsection{Lax pair in the discrete-time case and auxiliary variables}

Now, we discuss how to construct the time discretization of a given lattice system having the Lax pair $L_{n}$ and $M_{n}$. The natural discrete-time analogue of the linear system (2.1) is given by

$$
\Psi_{n+1}=L_{n}(\lambda) \Psi_{n}, \quad \widetilde{\Psi}_{n}=V_{n}(\lambda) \Psi_{n},
$$

where the tilde denotes the forward shift $(m \rightarrow m+1)$ in the discrete time coordinate $m \in \mathbb{Z}$. Here and hereafter, the dependence on $m$ is usually suppressed unless it is shifted. The compatibility condition of this overdetermined linear system is given by $[2,3,5,7,19,20]$

$$
\widetilde{L}_{n} V_{n}=V_{n+1} L_{n}
$$

which is (a fully discretized version of) the zero-curvature condition. Note that (2.6) can be rewritten as

$$
V_{n}=\widetilde{L}_{n}^{-1} V_{n+1} L_{n} \text { or } \widetilde{L}_{n} V_{n} L_{n}^{-1}=V_{n+1} .
$$

Following the work of Ablowitz and coworkers [2, 3, 5], the matrix $L_{n}(\lambda)$ is assumed to be the same as that in the semi-discrete case. Then, we look for a $V_{n}(\lambda)$ such that the zero-curvature condition (2.6) results in a closed system of partial difference equations providing a discrete-time analogue of the semi-discrete system.

For this purpose, we assume that the matrix $V_{n}$ has asymptotic behavior,

$$
V_{n}=I+h\left[M_{n}+O(h)\right],
$$

where $h$ is a sufficiently small (but nonzero) parameter independent of $\lambda$ and is considered the difference interval of time (cf. (2.2) and (2.6) $)$. More precisely, $h$ approximates the "true" step size of discrete time up to an $o(h)$ error. 
Moreover, we assume that $V_{n}(\lambda)$ has essentially the same $\lambda$-dependence as $I+h M_{n}(\lambda)$. Note, however, that $M_{n}(\lambda)$ can only be determined up to the addition of an $n$-independent scalar matrix (cf. (b)). Thus, this arbitrariness has to be taken into account in determining $V_{n}(\lambda)$; this corresponds to the freedom (nonuniqueness) of choosing the linear part of the time-discretized lattice system that determines the dispersion relation [3]. Moreover, some $n$-independent quantities (usually set as constants) in $M_{n}$ translate into $n$ dependent quantities in $V_{n}$, which typically constitute, up to the reformulation of the dependent variables, new auxiliary variables. Then, we specify appropriate boundary conditions for these new variables in $V_{n}$, which should retrieve the corresponding $n$-independent values in $M_{n}$. In fact, we usually assume "constant" boundary conditions for $V_{n}$,

$$
\lim _{n \rightarrow-\infty} V_{n}=\lim _{n \rightarrow+\infty} V_{n}=\text { finite. }
$$

However, the right-hand side is allowed to depend on the time variable $m \in \mathbb{Z}$. In the application of the inverse scattering method based on the Lax pair, we need to specify the boundary conditions for $L_{n}$ as $n \rightarrow \pm \infty$. In such a case, it is redundant to impose the boundary conditions on $V_{n}$ at both spatial ends as given in (2.9), and it is nontrivial that the redundant boundary conditions are compatible. In fact, it is sufficient to know only one of the two boundary values, $\lim _{n \rightarrow-\infty} V_{n}$ or $\lim _{n \rightarrow+\infty} V_{n}$. In the existing literature [1 3 , 6,7,19], it is hypothesized that these two limits indeed coincide; a preliminary consideration without using this hypothesis is given in [21]. In section 3, we demonstrate for specific examples that this is not a hypothesis but a verifiable fact.

Let us decompose $L_{n}(\lambda)$ into a sum of terms, each of which is the product of an $(n, m)$-independent scalar function of $\lambda$ and a $\lambda$-independent matrix, i.e.,

$$
L_{n}(\lambda)=\sum_{i=i_{\min }}^{i_{\max }} f_{i}(\lambda) L_{n}^{(i)} .
$$

The scalar functions $f_{i}(\lambda)\left(i_{\min } \leq i \leq i_{\max }\right)$ are linearly independent; typically, they are powers of $\lambda, e . g ., f_{i}(\lambda)=\lambda^{i}$. The nonzero elements of the matrices $L_{n}^{(i)}$ are classified into two types, that is, constants (or, at most, arbitrary functions of only one independent variable) and dynamical variables depending on both independent variables. We express the entire set of functionally independent dynamical variables in $L_{n}^{(i)}\left(i_{\min } \leq i \leq i_{\max }\right)$ as $\left\{\boldsymbol{l}_{n}\right\}$. The set of dynamical variables $\left\{\boldsymbol{v}_{n}\right\}$ is defined from $V_{n}(\lambda)$ in exactly the same way. Then, the zero-curvature condition (2.6) provides a (typically bilinear algebraic) system for $\left\{\widetilde{\boldsymbol{l}}_{n}, \boldsymbol{l}_{n}\right\}$ and $\left\{\boldsymbol{v}_{n}, \boldsymbol{v}_{n+1}\right\}$. In particular, this system contains a useful subsystem, that is, an ultralocal and linear system in 
$\left\{\boldsymbol{v}_{n}\right\}$, wherein the coefficients involve $\left\{\boldsymbol{l}_{n}\right\}$ and its shifts; this subsystem can also be derived by noting that (2.7) holds true as an identity in $\lambda$. We solve this subsystem to express a subset of $\left\{\boldsymbol{v}_{n}\right\}$ in terms of the remaining $\left\{\boldsymbol{v}_{n}\right\}$ as well as $\left\{\boldsymbol{l}_{n}\right\}$ and its shifts such as $\left\{\widetilde{\boldsymbol{l}}_{n}\right\}$ and $\left\{\boldsymbol{l}_{n-1}\right\}$. Thus, we can reduce the number of independent dynamical variables in $V_{n}$ while maintaining the ultralocality of $V_{n}$ with respect to $\left\{\boldsymbol{v}_{n}\right\}$. It remains to be verified that the zero-curvature condition (2.6) indeed provides a meaningful fully discrete system for the reduced set of dependent variables; it should define a consistent and unique time evolution for generic initial data under appropriate boundary conditions (cf. (2.9)).

\subsection{Fundamental "conservation laws" in the discrete- time case}

We consider the determinant of both sides of (2.6) to obtain the equality

$$
\left(\operatorname{det} \widetilde{L}_{n}\right)\left(\operatorname{det} V_{n}\right)=\left(\operatorname{det} L_{n}\right)\left(\operatorname{det} V_{n+1}\right) .
$$

This relation can be written more explicitly in the form of a discrete conservation law,

$$
\boldsymbol{\Delta}_{m}^{+} \log \left(\operatorname{det} L_{n}\right)=\boldsymbol{\Delta}_{n}^{+} \log \left(\operatorname{det} V_{n}\right) .
$$

Here, $\boldsymbol{\Delta}_{m}^{+}$is the forward difference operator in the time direction, i.e., $\boldsymbol{\Delta}_{m}^{+} f_{n}:=\widetilde{f}_{n}-f_{n}$. This is the discrete-time version of (2.3). Note that after cancelling the $m$ independent factor of $\operatorname{det} L_{n}$, the relation (2.10) should reduce to either

$$
\operatorname{det} V_{n}=\operatorname{det} V_{n+1}
$$

or

$$
\exp \left(\widetilde{\rho}_{n}\right) \operatorname{det} V_{n}=\exp \left(\rho_{n}\right) \operatorname{det} V_{n+1},
$$

where $\rho_{n}$ is a nontrivial conserved density. In the first case (2.11), one may speculate that the above conservation law may become the meaningless relation $\boldsymbol{\Delta}_{m}^{+}$(const.) $=\boldsymbol{\Delta}_{n}^{+}$(const.). However, in all the examples that require the introduction of auxiliary variables, this appears not to be the case; it is not immediately evident that the determinant of $V_{n}$ is $n$-independent, and thus the relation (2.10) still contains meaningful information. We employ a simplified but still ultralocal (with respect to $\left\{\boldsymbol{v}_{n}\right\}$ ) form of $V_{n}$, compute its determinant, and expand it with respect to $\lambda$ in the summed form

$$
\operatorname{det} V_{n}(\lambda)=\sum_{j=j_{\min }}^{j_{\max }} g_{j}(\lambda) a_{n}^{(j)}
$$


Here, $g_{j}(\lambda)\left(j_{\min } \leq j \leq j_{\max }\right)$ are linearly independent functions of $\lambda$, e.g., $g_{j}(\lambda)=\lambda^{2 j}$, and their coefficients $a_{n}^{(j)}$ are $\lambda$-independent functions of $\left\{\boldsymbol{v}_{n}\right\}$, $\left\{\boldsymbol{l}_{n}\right\}$, and the space/time shifts of $\left\{\boldsymbol{l}_{n}\right\}$. Substituting (2.13) into (2.11), we obtain the $n$-independence of the coefficients of $g_{j}(\lambda)$ for all $j$, i.e.,

$$
a_{n}^{(j)}=\lim _{n \rightarrow \pm \infty} a_{n}^{(j)}, \quad j_{\min } \leq j \leq j_{\max } .
$$

In the second case (2.12), which is more common than (2.11), the substitution of $(2.13)$ gives the set of relations

$$
\exp \left(\widetilde{\rho}_{n}\right) a_{n}^{(j)}=\exp \left(\rho_{n}\right) a_{n+1}^{(j)}, \quad j_{\min } \leq j \leq j_{\max } .
$$

Thus, there exist seemingly more than one flux $\log a_{n}^{(j)}$ associated with the same conserved density $\rho_{n}$, but the $a_{n}^{(j)}$ should coincide up to trivial proportionality factors. Indeed, calculating the ratio of the above equality for different values of $j$ on both sides, we obtain

$$
\frac{a_{n}^{\left(j_{1}\right)}}{a_{n}^{\left(j_{2}\right)}}=\lim _{n \rightarrow \pm \infty} \frac{a_{n}^{\left(j_{1}\right)}}{a_{n}^{\left(j_{2}\right)}}, \quad j_{\min } \leq j_{1} \neq j_{2} \leq j_{\max } .
$$

That is, the ratio $a_{n}^{\left(j_{\min }\right)}: \cdots: a_{n}^{\left(j_{\max }\right)}$ is independent of $n$. In both the above cases, the right-hand side of (2.14) or (2.15) is determined by the boundary conditions for $V_{n}$, in particular, the boundary values of the dependent variables contained in $V_{n}$; each right-hand side is set as a definite value independent of $n$ (cf. (2.9) ). This results in an "ultralocal" algebraic system for a subset of $\left\{\boldsymbol{v}_{n}\right\}$ that essentially constitutes the auxiliary variables; the number of independent unknowns is usually equal to that of the independent equalities so that this algebraic system is neither overdetermined nor underdetermined.

\subsection{Algebraic system for the auxiliary variables and local equations of motion}

We solve the obtained algebraic system for the auxiliary variables appearing in $V_{n}$ (and not in $M_{n}$ ). By eliminating all but one of the auxiliary variables, this system becomes a scalar algebraic equation in the remaining auxiliary variable; the other auxiliary variables are expressible in terms of the solution of this equation. The degree and complexity of this algebraic equation depend on the boundary conditions for $V_{n}$, which determine the dispersion relation of the time-discretized lattice system. In this paper, we mainly consider the case for a degree of two so that the equation is solvable by the quadratic 
formula. For sufficiently small $h$, referring to the prescribed behavior (2.8) of $V_{n}$, we can discard one of the two solutions as improper, and obtain a unique proper solution of the quadratic equation. In general, the larger the number of grid points defining the lattice system as well as the dispersion relation, the higher the degree of the algebraic equation determining the auxiliary variables. The higher-degree case can be interpreted as a composition of lower-degree cases; that is, the time evolution in the higher-degree case can be factorized into sequential applications of more elementary time evolutions. This point will be illustrated in section 3 . Once all the auxiliary variables have been expressed locally in terms of the original dependent variables that have already appeared in the semi-discrete case, we only have to substitute them into an appropriate subset of equations arising from the zero-curvature condition (2.6). Because of the use of the "conservation laws" (cf. (2.11) or (2.12) ), not all of the equations arising from (2.6) are independent and necessary any longer. We choose a minimal subset of these equations so that the substitution of the local expressions in the auxiliary variables produces the unique discrete-time evolution of the lattice system.

Last but not least, the above set of conservation laws used to determine the auxiliary variables can, in principle, be derived from the original system of partial difference equations resulting from the zero-curvature condition (2.6). However, in practice, this is an extremely difficult task; any computation conducted at the component level without following a set procedure is highly unlikely to arrive at the nontrivial conservation laws to be derived. Thus, our derivation performed at the matrix level using a determinantal formula and the spectral parameter is possibly the only way of obtaining them.

\subsection{Remarks on nonautonomous extensions}

Actually, the "step size" parameter $h$ introduced in (2.8) need not be a constant and can depend arbitrarily on the discrete time coordinate $m \in \mathbb{Z}$; in other words, the discrete-time flow involving an arbitrary parameter $h$ belongs to the same hierarchy, and we can specify any value of $h$ at every step of the time evolution. Indeed, this does not result in any essential difference in the subsequent computations, because the zero-curvature condition (2.6) involves equal-time $V_{n}$ only. In this way, we can obtain nonautonomous extensions of time-discretized lattice systems involving one arbitrary function of the discrete time (see, for example, 222 and references therein). Moreover, if the time discretization considered can be factorized into a composition of $M$ elementary time evolutions (cf. [6, 7, 19]), then it generally allows an extension involving $M$ arbitrary functions of time; however, we do not proactively discuss this possibility to avoid unnecessary confusion. From the 
modern point of view (cf. [20,23, 25]), a discrete-time flow can be identified with the spatial part of an auto-Bäcklund transformation of the original continuous-time flow or an auto-Bäcklund transformation of the whole hierarchy of continuous-time flows. Thus, the consistency of this nonautonomous ("nonuniform in time") extension can be understood as the commutativity of auto-Bäcklund transformations for different values of the Bäcklund parameter [26, 27], known as Bianchi's permutability theorem. Readers interested in Bäcklund transformations are referred to the Proceedings [28].

Bianchi's permutability often implies an ultralocal relation among the four solutions of the same system, called a nonlinear superposition formula; its derivation is based on the compatibility condition of two "different" time evolutions: $\widetilde{\Psi}=V\left(\lambda, h_{1}\right) \Psi$ and $\widehat{\Psi}=V\left(\lambda, h_{2}\right) \Psi$. If there exist two or more distinct one-parameter auto-Bäcklund transformations, then the matrix $V\left(\lambda, h_{2}\right)$ may be generalized to $W\left(\lambda, h_{2}\right)$. The nonlinear superposition formula motivates us to place the four solutions appropriately at the four vertices of a rectangle [15,16] and to assign each value of the Bäcklund parameter to each pair of parallel sides; this is often referred to as the Lamb diagram (see Fig. 3 in [26]). It is convenient to identify each value of the Bäcklund parameter with the length of each side, although values are not restricted to positive numbers. We can use this rectangle as an elementary cell defining two directions of new independent variables [15-17]. In fact, repeated applications of the auto-Bäcklund transformation at (generally) distinct values of the Bäcklund parameter generate a two-dimensional unequally spaced (in both directions) lattice in the quadrant (cf. Fig. 12 in [27] and the main figure in [29]), or even in the plane, with elementary cells of various sizes. With this understanding, we can reinterpret every nonlinear superposition formula as a fully discretized lattice system involving one arbitrary function of one independent variable and another arbitrary function of the other independent variable.

Thus, if we encounter a time discretization of an integrable lattice system that has the same form as a nonlinear superposition formula for some continuous/discrete integrable system, then it allows a natural nonautonomous extension involving two arbitrary functions originating from two values of the Bäcklund parameter. For example, the nonlinear superposition formula for the potential KdV hierarchy (cf. (14) or (16) in [30]) suggests a nonautonomous extension of a discrete potential KdV equation (cf. (5) and (6) in [31]; (5.16) in [32]; (68) in [33]), while the nonlinear superposition formula for the sine-Gordon equation (or the potential mKdV hierarchy) with simple sign handling implies a nonautonomous extension of the fully discretized 
sine-Gordon equation [20,34] given by

$$
\tan \left(\frac{u_{n+1, m+1}+u_{n, m}}{4}\right)=\frac{f(n)+g(m)}{f(n)-g(m)} \tan \left(\frac{u_{n+1, m}+u_{n, m+1}}{4}\right),
$$

or equivalently,

$$
\begin{aligned}
& \frac{f(n)}{g(m)} \sin \left(\frac{u_{n+1, m+1}-u_{n+1, m}-u_{n, m+1}+u_{n, m}}{4}\right) \\
& =\sin \left(\frac{u_{n+1, m+1}+u_{n+1, m}+u_{n, m+1}+u_{n, m}}{4}\right) .
\end{aligned}
$$

If we rescale/redefine the arbitrary functions and variables as $f(n)=(4 / \Delta) F(n \Delta)$ and $g(m)=h G(m h)$, and $u_{n, m}=u(n \Delta, m h), n \Delta=: x$, and $m h=: t$, respectively, then the continuous limit $\Delta, h \rightarrow 0$ reduces (2.17) to the variablecoefficient sine-Gordon equation

$$
\frac{F(x)}{G(t)} u_{x t}=\sin u
$$

which is obviously equivalent to the constant-coefficient sine-Gordon equation $u_{X T}=\sin u$. Thus, this type of nonautonomous extension in the discrete case is thought to be a vestige of coordinate transformations in the continuous case that do not mix the two independent variables.

It is still unclear whether any of the time-discretized lattice systems obtained in the next section in their present form can be identified with a nonlinear superposition formula. Alternatively, we propose an intriguing procedure for constructing fully discrete nonautonomous systems involving two arbitrary functions; this procedure is closely related to the property of three-dimensional consistency [35-37]. It is assumed that $L_{n}(\lambda)$ is ultralocal in the set of dynamical variables $\left\{\boldsymbol{l}_{n}\right\}$, while $V_{n}(\lambda, h)$ can be written in terms of $\left\{\boldsymbol{l}_{n-1}, \widetilde{\boldsymbol{l}}_{n}\right\}$ or $\left\{\widetilde{\boldsymbol{l}}_{n-1}, \boldsymbol{l}_{n}\right\}$. We only consider the former case, because the latter case can be dealt with in a similar manner. Then, the linear problem (2.5) with the "step size" $h$ set as $h=h_{1}$ and $h=h_{2}$ respectively implies the relations

$$
\widetilde{\Psi}_{n}=N_{n}^{(1)} \Psi_{n-1}, \quad N_{n}^{(1)}:=V_{n}\left(\lambda, h_{1}\right) L_{n-1}(\lambda),
$$

and

$$
\widehat{\Psi}_{n}=N_{n}^{(2)} \Psi_{n-1}, \quad N_{n}^{(2)}:=V_{n}\left(\lambda, h_{2}\right) L_{n-1}(\lambda) .
$$

Here, the newly introduced matrix $N_{n}^{(1)}$ connects the values of $\Psi$ at two lattice points $(n-1, m)$ and $(n, m+1)$, and depends only on the dynamical variables at these points, namely, $\left\{\boldsymbol{l}_{n-1}, \widetilde{\boldsymbol{l}}_{n}\right\}$. Similarly, $N_{n}^{(2)}$ connects the 
two points $(n-1, m)$ and $\left(n, m+1^{\prime}\right)$, and depends on the dynamical variables at these points: $\left\{\boldsymbol{l}_{n-1}, \widehat{\boldsymbol{l}}_{n}\right\}$. Note that $\left(n, m+1^{\prime}\right)$ can be identified with $(n, m+1)$ only if $h_{1}=h_{2}$. The compatibility condition of (2.18) is given by a "new" zero-curvature equation,

$$
\widetilde{N}_{n+1}^{(2)} N_{n}^{(1)}=\widehat{N}_{n+1}^{(1)} N_{n}^{(2)} .
$$

Thus, the substitution of the explicit forms of the matrices $N_{n}^{(1)}\left(\left\{\boldsymbol{l}_{n-1}, \widetilde{\boldsymbol{l}}_{n}\right\}, h_{1}\right)$ and $N_{n}^{(2)}\left(\left\{\boldsymbol{l}_{n-1}, \widehat{\boldsymbol{l}}_{n}\right\}, h_{2}\right)$ into this equation should provide a closed system for the dynamical variables at the four lattice points

$$
\boldsymbol{l}_{n-1}, \widetilde{\boldsymbol{l}}_{n}, \widehat{\boldsymbol{l}}_{n}, \widehat{\widetilde{\boldsymbol{l}}}_{n+1}\left(=\widetilde{\boldsymbol{l}}_{n+1}\right) .
$$

We can regard the corresponding parallelogram as defining two directions of new independent variables, so that the system now involves two arbitrary functions originating from $h_{1}$ and $h_{2}$.

\section{Examples}

In this section, we apply the general method for time discretization in section 2 to five important examples: the Toda lattice, the Ablowitz-Ladik lattice (semi-discrete NLS), the Volterra lattice, the modified Volterra lattice, and the lattice Heisenberg ferromagnet model.

We are only interested in time discretizations that can accurately approximate the continuous-time dynamics in the small-value range of the "step size" parameter $h$. This implies that the discrete-time Lax matrix $V_{n}$ allows the asymptotic expansion with respect to $h$ given in (2.8); in particular, $\left(V_{n}-I\right) / h$ does not involve $O(1 / h)$ terms. This requirement plays a crucial role in obtaining suitable local expressions for the auxiliary variables appearing in $V_{n}$.

The parameter $h$ is generally assumed to be nonzero; alternatively, one can allow the case $h=0$ as the trivial identity mapping $\widetilde{\boldsymbol{l}}_{n}=\boldsymbol{l}_{n}$.

\subsection{The Toda lattice in Flaschka-Manakov coordinates}

We consider the Toda lattice written in Flaschka-Manakov coordinates [911:

$$
u_{n, t}=u_{n}\left(v_{n}-v_{n-1}\right), \quad v_{n, t}=u_{n+1}-u_{n} .
$$

The parametrization

$$
u_{n}=\mathrm{e}^{x_{n}-x_{n-1}}, \quad v_{n}=x_{n, t},
$$


enables the system (3.1) to be rewritten as the Newtonian equations of motion for the Toda lattice,

$$
x_{n, t t}=\mathrm{e}^{x_{n+1}-x_{n}}-\mathrm{e}^{x_{n}-x_{n-1}} .
$$

The Lax pair for the Toda lattice (3.1) in Flaschka-Manakov coordinates is given by [1]

$$
\begin{aligned}
L_{n} & =\left[\begin{array}{cc}
\lambda+v_{n} & u_{n} \\
-1 & 0
\end{array}\right], \\
M_{n} & =\left[\begin{array}{cc}
0 & -u_{n} \\
1 & \lambda+v_{n-1}
\end{array}\right] .
\end{aligned}
$$

Indeed, the substitution of (3.3) into the zero-curvature condition (2.2) results in (3.1).

Let us move to the discrete-time case. A comprehensive overview of the existing results on time discretizations of the Toda lattice is given in $\S 3.22$ and $\S 5.11$ of [1], thus we do not repeat it here. In view of the zero-curvature condition (2.6), we assume the Lax matrix $V_{n}$ of the following form:

$$
V_{n}=I+h\left[\begin{array}{cc}
-\lambda a+\alpha_{n} & -a u_{n}-\widetilde{u}_{n} b_{n} \\
a+b_{n} & \lambda b_{n}+\alpha_{n-1}+a v_{n-1}+b_{n} v_{n-1}
\end{array}\right] .
$$

Here, $\alpha_{n}$ and $b_{n}$ are auxiliary variables. The zero-curvature condition (2.6) for the Lax pair (3.3a) and (3.4) amounts to the following system of partial difference equations:

$$
\left\{\begin{array}{l}
\frac{1}{h}\left(\widetilde{u}_{n}-u_{n}\right)=\alpha_{n+1} u_{n}-\widetilde{u}_{n} \alpha_{n-1}+\widetilde{v}_{n}\left(a u_{n}+\widetilde{u}_{n} b_{n}\right)-\left(a \widetilde{u}_{n}+\widetilde{u}_{n} b_{n}\right) v_{n-1}, \\
\frac{1}{h}\left(\widetilde{v}_{n}-v_{n}\right)=\alpha_{n+1} v_{n}-\widetilde{v}_{n} \alpha_{n}+a\left(u_{n+1}-\widetilde{u}_{n}\right)+\widetilde{u}_{n+1} b_{n+1}-\widetilde{u}_{n} b_{n}, \\
\alpha_{n}-\alpha_{n+1}=a\left(\widetilde{v}_{n}-v_{n}\right), \\
\widetilde{u}_{n} b_{n}=b_{n+1} u_{n} .
\end{array}\right.
$$

The general form (3.5) of the time-discretized Toda lattice is integrable for matrix-valued dependent variables, but in the following, we consider only the case of scalar dependent variables. In view of (2.9) and (3.2), we impose the following boundary conditions for $u_{n}, v_{n}, \alpha_{n}$, and $b_{n}$ :

$$
\lim _{n \rightarrow \pm \infty} u_{n}=1, \quad \lim _{n \rightarrow \pm \infty} v_{n}=0, \quad \lim _{n \rightarrow \pm \infty} \alpha_{n}=0, \quad \lim _{n \rightarrow \pm \infty} b_{n}=b .
$$

The boundary value of $\alpha_{n}$ is set as zero by redefining $h$. To be precise, the boundary conditions (3.6) contain redundant information. Indeed, it can 
be shown that the auxiliary variables have the same limit values for $n \rightarrow-\infty$ and $n \rightarrow+\infty$. Therefore, it is sufficient to assume either $\lim _{n \rightarrow-\infty}\left(\alpha_{n}, b_{n}\right)=(0, b)$ or $\lim _{n \rightarrow+\infty}\left(\alpha_{n}, b_{n}\right)=(0, b)$. In the continuum limit of time $h \rightarrow 0$, the auxiliary variables reduce to constants, i.e., $\alpha_{n} \rightarrow 0$ and $b_{n} \rightarrow b$. Thus, in this limit, the time discretization (3.5) reduces to

$$
u_{n, t}=(a+b) u_{n}\left(v_{n}-v_{n-1}\right), \quad v_{n, t}=(a+b)\left(u_{n+1}-u_{n}\right),
$$

which is, if $a+b \neq 0$, equivalent to the Toda lattice (3.1) up to a redefinition of $t$. Note that in the case $a+b=0$, (3.5) has the trivial solution $\widetilde{u}_{n}=u_{n}$, $\widetilde{v}_{n}=v_{n}, \alpha_{n}=0, b_{n}=b$.

The determinant of the $2 \times 2$ Lax matrix $L_{n}$ (3.3a) can be immediately computed as det $L_{n}=u_{n}$. Using the recurrence formula for $\alpha_{n}$ in (3.5), we can rewrite the Lax matrix $V_{n}$ (3.4) in an ultralocal form with respect to the auxiliary variables $\alpha_{n}$ and $b_{n}$. Thus, its $2 \times 2$ determinant is computed as

$$
\begin{aligned}
& \operatorname{det} V_{n}(\lambda) \\
= & {\left[1+h\left(-\lambda a+\alpha_{n}\right)\right]\left[1+h\left(\lambda b_{n}+\alpha_{n}+a \widetilde{v}_{n-1}+b_{n} v_{n-1}\right)\right] } \\
& +h^{2}\left(a u_{n}+\widetilde{u}_{n} b_{n}\right)\left(a+b_{n}\right) \\
= & -\lambda^{2} h^{2} a b_{n}-\lambda h\left[\left(a-b_{n}\right)\left(1+h \alpha_{n}\right)+h a^{2} \widetilde{v}_{n-1}+h a b_{n} v_{n-1}\right] \\
& +\left(1+h \alpha_{n}\right)^{2}+\left(1+h \alpha_{n}\right)\left(h a \widetilde{v}_{n-1}+h b_{n} v_{n-1}\right)+h^{2}\left(a u_{n}+\widetilde{u}_{n} b_{n}\right)\left(a+b_{n}\right) .
\end{aligned}
$$

Therefore, the equality (2.10) combined with the boundary conditions (3.6) (or the streamlined version as stated above) implies the set of relations

$$
\left\{\begin{array}{l}
b_{n}=b \Lambda_{n} \\
\left(a-b_{n}\right)\left(1+h \alpha_{n}\right)+h a^{2} \widetilde{v}_{n-1}+h a b_{n} v_{n-1}=(a-b) \Lambda_{n} \\
\left(1+h \alpha_{n}\right)^{2}+\left(1+h \alpha_{n}\right)\left(h a \widetilde{v}_{n-1}+h b_{n} v_{n-1}\right) \\
+h^{2}\left(a u_{n}+\widetilde{u}_{n} b_{n}\right)\left(a+b_{n}\right)=\left[1+h^{2}(a+b)^{2}\right] \Lambda_{n} .
\end{array}\right.
$$

Here, $\Lambda_{n}$ is the quantity that satisfies the recurrence formula

$$
\widetilde{u}_{n} \Lambda_{n}=u_{n} \Lambda_{n+1}
$$

and has the normalized boundary value, $\lim _{n \rightarrow \pm \infty} \Lambda_{n}=1$. More precisely, only one of the two conditions $\lim _{n \rightarrow-\infty} \Lambda_{n}=1$ and $\lim _{n \rightarrow+\infty} \Lambda_{n}=1$ is required, in accordance with the boundary conditions for $\alpha_{n}$ and $b_{n}$. Then, the other condition can be confirmed. Thus, $\Lambda_{n}$ can be written explicitly (but globally) as

$$
\Lambda_{n}=\prod_{j=-\infty}^{n-1} \frac{\widetilde{u}_{j}}{u_{j}}=\prod_{j=n}^{+\infty} \frac{u_{j}}{\widetilde{u}_{j}} .
$$


Note that the first equality in (3.7) is valid even if $a=0$ (cf. (3.5)). The algebraic system (3.7) essentially comprises two nontrivial relations containing the two unknowns, $\alpha_{n}$ and $\Lambda_{n}$.

It is rather cumbersome to identify the dispersion relation of (3.5) from its linear leading part (cf. subsection 3.2). Instead, we can compute the asymptotic form of the Lax matrix $V_{n}$ (3.4) as $n \rightarrow-\infty$ or $n \rightarrow+\infty$ and consider its factorization (cf. [1, 6, 7, 19]). The results imply that the cases $a=0$ and $b=0$ form the basis for the general case; that is, the time evolution for general $a$ and $b$ is equivalent to the order-independent composition of two time evolutions corresponding to $a=0$ and $b=0$, respectively. In addition, these two cases are related to each other through the time reflection, as we will see below.

Now, we consider the two fundamental cases: $a=0$ or $b=0$.

- The case $a=0, b \neq 0$.

In this case, the auxiliary variable $\alpha_{n}$ vanishes. Thus, the discrete-time system (3.5) reduces to (cf. (3.6) in [38])

$$
\left\{\begin{array}{l}
\frac{1}{h}\left(\widetilde{u}_{n}-u_{n}\right)=b \Lambda_{n+1} u_{n}\left(\widetilde{v}_{n}-v_{n-1}\right), \\
\frac{1}{h}\left(\widetilde{v}_{n}-v_{n}\right)=b \Lambda_{n+1}\left(\widetilde{u}_{n+1}-u_{n}\right), \\
\widetilde{u}_{n} \Lambda_{n}=u_{n} \Lambda_{n+1}, \quad \lim _{n \rightarrow-\infty} \Lambda_{n}=1 \text { or } \lim _{n \rightarrow+\infty} \Lambda_{n}=1 .
\end{array}\right.
$$

The algebraic system (3.7) simplifies to a quadratic equation in $\Lambda_{n}$,

$$
(h b)^{2} \widetilde{u}_{n} \Lambda_{n}^{2}-\left[1+(h b)^{2}-h b v_{n-1}\right] \Lambda_{n}+1=0 .
$$

The asymptotic behavior (2.8) of the Lax matrix $V_{n}$ implies that the proper solution of (3.10) is given by

$$
\left[1+(h b)^{2}\right] \Lambda_{n}=\frac{2}{1-\epsilon v_{n-1}+\sqrt{\left(1-\epsilon v_{n-1}\right)^{2}-4 \epsilon^{2} \widetilde{u}_{n}}}
$$

where $\epsilon:=h b /\left[1+(h b)^{2}\right]$. Thus, if $h b \in \mathbb{R}$, then $-1 / 2 \leq \epsilon \leq 1 / 2$. In this case, the local expression (3.11) is valid only if $-1<h b<1$, which covers the range $-1 / 2<\epsilon<1 / 2$. The borderline cases $h b= \pm 1$ are excluded from our consideration. If $(h b)^{2}>1$, (3.11) is inconsistent with the boundary conditions, and the other solution of (3.10) should be adopted. In any case, the boundary conditions for $u_{n}$ and $v_{n}$ imply that $\lim _{n \rightarrow-\infty} \Lambda_{n}=\lim _{n \rightarrow+\infty} \Lambda_{n}=1$. 
Substituting (3.11) into the first and second equations in (3.9), we obtain a time discretization of the Toda lattice (3.1) in the local form,

$$
\left\{\begin{array}{l}
\frac{\widetilde{u}_{n}-u_{n}}{\epsilon}=\frac{2 u_{n}\left(\widetilde{v}_{n}-v_{n-1}\right)}{1-\epsilon v_{n}+\sqrt{\left(1-\epsilon v_{n}\right)^{2}-4 \epsilon^{2} \widetilde{u}_{n+1}}} \\
\frac{2\left(\widetilde{u}_{n+1}-u_{n}\right)}{\epsilon}=\frac{\widetilde{v}_{n}-v_{n}}{1-\epsilon v_{n}+\sqrt{\left(1-\epsilon v_{n}\right)^{2}-4 \epsilon^{2} \widetilde{u}_{n+1}}}
\end{array}\right.
$$

Under the boundary conditions $\lim _{n \rightarrow \pm \infty} u_{n}=1$ and $\lim _{n \rightarrow \pm \infty} v_{n}=0, \widetilde{v}_{n}$ is determined from $u_{n}, v_{n}$, and $\widetilde{u}_{n+1}$, and subsequently, $\widetilde{u}_{n}$ is determined from $v_{n-1}, u_{n}, v_{n}, \widetilde{v}_{n}$, and $\widetilde{u}_{n+1}$. If $h b \in \mathbb{R}$, the $u_{n}$ are nonzero and real-valued, and the $v_{n}$ are real-valued at the initial time, then (3.9) implies that the auxiliary variable $\Lambda_{n}$ is always real-valued. Consequently, the discriminant of the quadratic equation (3.10) must be nonnegative. Thus, as long as the amplitudes of $u_{n}-1$ and $v_{n}$ are sufficiently small and their effects can be regarded as perturbations, the real-valuedness of $u_{n}$ and $v_{n}$ is preserved under the time evolution of the discrete-time Toda lattice (3.12) for $-1 / 2<\epsilon<1 / 2$.

To express the backward time evolution explicitly, we only have to replace $\Lambda_{n+1}$ in the first and second equations of (3.9) with $\Lambda_{n} \widetilde{u}_{n} / u_{n}$ and then substitute the local expression (3.11). The resulting system is

$$
\left\{\begin{array}{l}
\frac{\widetilde{u}_{n}-u_{n}}{\epsilon}=\frac{2 \widetilde{u}_{n}\left(\widetilde{v}_{n}-v_{n-1}\right)}{1-\epsilon v_{n-1}+\sqrt{\left(1-\epsilon v_{n-1}\right)^{2}-4 \epsilon^{2} \widetilde{u}_{n}}}, \\
\frac{\widetilde{v}_{n}-v_{n}}{\epsilon}=\frac{\widetilde{u}_{n}}{u_{n}} \times \frac{2\left(\widetilde{u}_{n+1}-u_{n}\right)}{1-\epsilon v_{n-1}+\sqrt{\left(1-\epsilon v_{n-1}\right)^{2}-4 \epsilon^{2} \widetilde{u}_{n}}} .
\end{array}\right.
$$

Using the first equation, the second equation in (3.13) can also be written as

$$
\frac{\widetilde{v}_{n}-v_{n}}{\epsilon}=\frac{2\left(\widetilde{u}_{n+1}-u_{n}\right)}{1-\epsilon\left(2 \widetilde{v}_{n}-v_{n-1}\right)+\sqrt{\left(1-\epsilon v_{n-1}\right)^{2}-4 \epsilon^{2} \widetilde{u}_{n}}} .
$$

- The case $b=0, a \neq 0$.

In this case, the auxiliary variable $b_{n}$ vanishes. Thus, the discrete-time system (3.5) reduces to

$$
\left\{\begin{array}{l}
\frac{1}{h}\left(1+h \alpha_{n}+h a \widetilde{v}_{n-1}\right)\left(\widetilde{u}_{n}-u_{n}\right)=a u_{n}\left(v_{n}-\widetilde{v}_{n-1}\right), \\
\frac{1}{h}\left(1+h \alpha_{n}+h a v_{n}\right)\left(\widetilde{v}_{n}-v_{n}\right)=a\left(u_{n+1}-\widetilde{u}_{n}\right), \\
\alpha_{n}-\alpha_{n+1}=a\left(\widetilde{v}_{n}-v_{n}\right), \quad \lim _{n \rightarrow-\infty} \alpha_{n}=0 \text { or } \lim _{n \rightarrow+\infty} \alpha_{n}=0 .
\end{array}\right.
$$


The algebraic system (3.7) simplifies to

$$
\left\{\begin{array}{l}
1+h \alpha_{n}+h a \widetilde{v}_{n-1}=\Lambda_{n}, \\
\left(1+h \alpha_{n}\right)^{2}+\left(1+h \alpha_{n}\right) h a \widetilde{v}_{n-1}+(h a)^{2} u_{n}=\left[1+(h a)^{2}\right] \Lambda_{n} .
\end{array}\right.
$$

Note that, using $\Lambda_{n}$ instead of $\alpha_{n}$, system (3.14) with (3.8) and (3.15) can be identified with the discrete-time Toda lattice given in $\S 3.8$ of [1] (also see [39]).

By eliminating $\Lambda_{n}$, (3.15) reduces to a quadratic equation in $1+h \alpha_{n}$,

$$
\left(1+h \alpha_{n}\right)^{2}-\left[1+(h a)^{2}-h a \widetilde{v}_{n-1}\right]\left(1+h \alpha_{n}\right)+(h a)^{2} u_{n}-\left[1+(h a)^{2}\right] h a \widetilde{v}_{n-1}=0 .
$$

The proper solution of this quadratic equation is given by

$$
\frac{1+h \alpha_{n}}{1+(h a)^{2}}=\frac{1-\delta \widetilde{v}_{n-1}+\sqrt{\left(1+\delta \widetilde{v}_{n-1}\right)^{2}-4 \delta^{2} u_{n}}}{2}
$$

where $\delta:=h a /\left[1+(h a)^{2}\right]$. Thus, if $h a \in \mathbb{R}$, then $-1 / 2 \leq \delta \leq 1 / 2$. In this case, the local expression (3.16) is valid only if $-1<h a<1$, which covers the range $-1 / 2<\delta<1 / 2$. The borderline cases $h a= \pm 1$ are excluded from our consideration. If $(h a)^{2}>1,(3.16)$ is inconsistent with the boundary conditions, and the other solution of the quadratic equation should be employed. In any case, the boundary conditions for $u_{n}$ and $v_{n}$ imply that $\lim _{n \rightarrow-\infty} \alpha_{n}=\lim _{n \rightarrow+\infty} \alpha_{n}=0$, and consequently, $\lim _{n \rightarrow-\infty} \Lambda_{n}=\lim _{n \rightarrow+\infty} \Lambda_{n}=1$.

Substituting the local expression (3.16) into the first and second equations of (3.14), we obtain a time discretization of the Toda lattice (3.1),

$$
\left\{\begin{array}{l}
\frac{\widetilde{u}_{n}-u_{n}}{\delta}=\frac{2 u_{n}\left(v_{n}-\widetilde{v}_{n-1}\right)}{1+\delta \widetilde{v}_{n-1}+\sqrt{\left(1+\delta \widetilde{v}_{n-1}\right)^{2}-4 \delta^{2} u_{n}}} \\
\frac{2\left(u_{n+1}-\widetilde{u}_{n}\right)}{\delta}=\frac{\widetilde{v}_{n}-v_{n}}{1+\delta\left(2 v_{n}-\widetilde{v}_{n-1}\right)+\sqrt{\left(1+\delta \widetilde{v}_{n-1}\right)^{2}-4 \delta^{2} u_{n}}}
\end{array}\right.
$$

Under the boundary conditions $\lim _{n \rightarrow \pm \infty} u_{n}=1$ and $\lim _{n \rightarrow \pm \infty} v_{n}=0, \widetilde{u}_{n}$ is determined from $\widetilde{v}_{n-1}, u_{n}$, and $v_{n}$, and subsequently, $\widetilde{v}_{n}$ is determined from $\widetilde{v}_{n-1}, \widetilde{u}_{n}, u_{n}, v_{n}$, and $u_{n+1}$. As long as $u_{n}$ and $v_{n}$ can be regarded as perturbations around the boundary values 1 and 0 , their real-valuedness is preserved under the time evolution of the discrete-time Toda lattice (3.17) for $-1 / 2<\delta<1 / 2$.

To obtain the backward time evolution explicitly, we rewrite $\alpha_{n}$ in the first and second equations of (3.14) as $\alpha_{n+1}+a\left(\widetilde{v}_{n}-v_{n}\right)$ and then substitute 
the local expression (3.16). The resulting system is

$$
\left\{\begin{array}{l}
\frac{\widetilde{u}_{n}-u_{n}}{\delta}=\frac{2 \widetilde{u}_{n}\left(v_{n}-\widetilde{v}_{n-1}\right)}{1+\delta \widetilde{v}_{n}+\sqrt{\left(1+\delta \widetilde{v}_{n}\right)^{2}-4 \delta^{2} u_{n+1}}}, \\
\frac{2\left(u_{n+1}-\widetilde{u}_{n}\right)}{\delta}=\frac{\widetilde{v}_{n}-v_{n}}{1+\delta \widetilde{v}_{n}+\sqrt{\left(1+\delta \widetilde{v}_{n}\right)^{2}-4 \delta^{2} u_{n+1}}}
\end{array}\right.
$$

It is easy to see that (3.17) is equivalent to (3.13) through the time reflection and the identification $\delta \leftrightarrow-\epsilon$. In the same manner, (3.18) can be identified with (3.12) through the time reflection. Thus, the forward/backward time evolution in the case $b=0$ corresponds to the backward/forward time evolution in the case $a=0$, up to a redefinition of the parameters.

\subsection{The Ablowitz-Ladik lattice}

In this subsection, we discuss the time discretization of the Ablowitz-Ladik lattice, which we consider to be the most instructive example to illustrate our general method. The (nonreduced form of the) Ablowitz-Ladik lattice is

$$
\left\{\begin{array}{l}
q_{n, t}-a q_{n+1}+b q_{n-1}+(a-b) q_{n}+a q_{n+1} r_{n} q_{n}-b q_{n} r_{n} q_{n-1}=0 \\
r_{n, t}-b r_{n+1}+a r_{n-1}+(b-a) r_{n}+b r_{n+1} q_{n} r_{n}-a r_{n} q_{n} r_{n-1}=0
\end{array}\right.
$$

and its Lax pair (cf. (2.1) and (2.2) ) is given by

$$
\begin{aligned}
L_{n} & =\lambda\left[\begin{array}{ll}
1 & \\
& 0
\end{array}\right]+\left[\begin{array}{cc}
0 & q_{n} \\
r_{n} & 0
\end{array}\right]+\frac{1}{\lambda}\left[\begin{array}{cc}
0 & \\
& 1
\end{array}\right]=\left[\begin{array}{cc}
\lambda & q_{n} \\
r_{n} & \frac{1}{\lambda}
\end{array}\right], \\
M_{n} & =\left[\begin{array}{cc}
\lambda^{2} a-a\left(1+q_{n} r_{n-1}\right) & \lambda a q_{n}+\frac{b}{\lambda} q_{n-1} \\
\lambda a r_{n-1}+\frac{b}{\lambda} r_{n} & \frac{b}{\lambda^{2}}-b\left(1+r_{n} q_{n-1}\right)
\end{array}\right] .
\end{aligned}
$$

Here, the free parameters $a$ and $b$ are usually set as constants, but they can depend on the time variable $t$ in an arbitrary manner, namely, $a:=a(t)$, $b:=b(t)$. The familiar form of the Ablowitz-Ladik lattice is obtained from (3.19) through the reduction of the complex conjugate: $b=a^{*}, r_{n}=\sigma q_{n}^{*}$, where $\sigma$ is a real constant. The case where $a$ and $b$ are purely imaginary leads to an integrable semi-discretization of the NLS equation. Taking into account the $\lambda$-dependence of the Lax matrix $M_{n}$ (3.20b), we look for a Lax matrix $V_{n}$ with the following dependence on $\lambda$ :

$$
V_{n}(\lambda)=I+h\left\{\lambda^{2} V_{n}^{(2)}+\lambda V_{n}^{(1)}+V_{n}^{(0)}+\frac{1}{\lambda} V_{n}^{(-1)}+\frac{1}{\lambda^{2}} V_{n}^{(-2)}\right\} .
$$


Here, $V_{n}^{(j)}(j=2,0,-2)$ are diagonal matrices, while $V_{n}^{(j)}(j=1,-1)$ are off-diagonal matrices. The nonzero parameter $h$ may depend on the discrete time coordinate $m \in \mathbb{Z}$. Substituting (3.20a) and (3.21) into (2.6), we find that the matrix $V_{n}$ should assume the form

$$
V_{n}=I+h\left[\begin{array}{c|c}
\lambda^{2} a+\widetilde{q}_{n} c_{n} r_{n-1} & \lambda\left(a q_{n}-\widetilde{q}_{n} c_{n}\right) \\
+\alpha_{n}+\frac{1}{\lambda^{2}} d_{n} & +\frac{1}{\lambda}\left(b \widetilde{q}_{n-1}-d_{n} q_{n-1}\right) \\
\hline \lambda\left(a \widetilde{r}_{n-1}-c_{n} r_{n-1}\right) & \lambda^{2} c_{n}+\widetilde{r}_{n} d_{n} q_{n-1} \\
+\frac{1}{\lambda}\left(b r_{n}-\widetilde{r}_{n} d_{n}\right) & +\beta_{n}+\frac{b}{\lambda^{2}}
\end{array}\right] .
$$

Here, $\alpha_{n}, \beta_{n}, c_{n}$, and $d_{n}$ are auxiliary variables. The zero-curvature condition (2.6) is equivalent to the following system of partial difference equations:

$$
\left\{\begin{aligned}
& \frac{1}{h}\left(\widetilde{q}_{n}-q_{n}\right)-a q_{n+1}+b \widetilde{q}_{n-1}-\alpha_{n+1} q_{n}+\widetilde{q}_{n} \beta_{n} \\
&+\widetilde{q}_{n+1} c_{n+1}\left(1-r_{n} q_{n}\right)-\left(1-\widetilde{q}_{n} \widetilde{r}_{n}\right) d_{n} q_{n-1}=0 \\
& \frac{1}{h}\left(\widetilde{r}_{n}-r_{n}\right)-b r_{n+1}+a \widetilde{r}_{n-1}-\beta_{n+1} r_{n}+\widetilde{r}_{n} \alpha_{n} \\
&+\widetilde{r}_{n+1} d_{n+1}\left(1-q_{n} r_{n}\right)-\left(1-\widetilde{r}_{n} \widetilde{q}_{n}\right) c_{n} r_{n-1}=0 \\
& \alpha_{n+1}-\alpha_{n}= a\left(\widetilde{q}_{n} \widetilde{r}_{n-1}-q_{n+1} r_{n}\right) \\
& \beta_{n+1}-\beta_{n}= b\left(\widetilde{r}_{n} \widetilde{q}_{n-1}-r_{n+1} q_{n}\right), \\
&\left(1-\widetilde{r}_{n} \widetilde{q}_{n}\right) c_{n}=c_{n+1}\left(1-r_{n} q_{n}\right) \\
&\left(1-\widetilde{q}_{n} \widetilde{r}_{n}\right) d_{n}=d_{n+1}\left(1-q_{n} r_{n}\right) .
\end{aligned}\right.
$$

Actually, the general nonreduced form (3.23) of the time-discretized AblowitzLadik lattice is integrable for matrix-valued dependent variables. However, in this paper, we consider only the case of scalar dependent variables. In addition to (2.9), we impose rapidly decaying boundary conditions for $q_{n}$ and $r_{n}$, that is,

$$
\lim _{n \rightarrow \pm \infty} q_{n}=\lim _{n \rightarrow \pm \infty} r_{n}=0, \quad \lim _{n \rightarrow \pm \infty}\left(\alpha_{n}, \beta_{n}, c_{n}, d_{n}\right)=(\alpha, \beta, c, d) .
$$

Similarly to the semi-discrete case, the parameters $a, b, \alpha, \beta, c$, and $d$ are allowed to depend on the discrete time coordinate $m \in \mathbb{Z}$. Thus, the time dependence of $h$ can be absorbed by $a, b, \alpha_{n}, \beta_{n}, c_{n}$, and $d_{n}$. For the same reason, it is possible to set $h$ as unity, but we prefer to leave it as a small parameter, usually $0<|h| \ll 1$. To be precise, the boundary conditions (3.24) contain redundant information. Indeed, it can be shown that the auxiliary variables have the same limit values for $n \rightarrow-\infty$ and $n \rightarrow+\infty$. Therefore, it is sufficient to assume either $\lim _{n \rightarrow-\infty}\left(\alpha_{n}, \beta_{n}, c_{n}, d_{n}\right)=(\alpha, \beta, c, d)$ or 
$\lim _{n \rightarrow+\infty}\left(\alpha_{n}, \beta_{n}, c_{n}, d_{n}\right)=(\alpha, \beta, c, d)$. The last four relations in (3.23) imply the following global expressions for the auxiliary variables $\alpha_{n}, \beta_{n}, c_{n}$, and $d_{n}$ in terms of $q_{n}$ and $r_{n}$ [1, 3, 5, 2, 19]:

$$
\begin{aligned}
\alpha_{n} & =\alpha-a q_{n} r_{n-1}+a \sum_{j=-\infty}^{n-1}\left(\widetilde{q}_{j} \widetilde{r}_{j-1}-q_{j} r_{j-1}\right) \\
& =\alpha-a \widetilde{q}_{n} \widetilde{r}_{n-1}-a \sum_{j=n+1}^{+\infty}\left(\widetilde{q}_{j} \widetilde{r}_{j-1}-q_{j} r_{j-1}\right), \\
\beta_{n} & =\beta-b r_{n} q_{n-1}+b \sum_{j=-\infty}^{n-1}\left(\widetilde{r}_{j} \widetilde{q}_{j-1}-r_{j} q_{j-1}\right) \\
& =\beta-b \widetilde{r}_{n} \widetilde{q}_{n-1}-b \sum_{j=n+1}^{+\infty}\left(\widetilde{r}_{j} \widetilde{q}_{j-1}-r_{j} q_{j-1}\right), \\
c_{n} & =c \prod_{j=-\infty}^{n-1} \frac{1-\widetilde{q}_{j} \widetilde{r}_{j}}{1-q_{j} r_{j}}=c \prod_{j=n}^{+\infty} \frac{1-q_{j} r_{j}}{1-\widetilde{q}_{j} \widetilde{r}_{j}}, \\
d_{n} & =d \prod_{j=-\infty}^{n-1} \frac{1-\widetilde{q}_{j} \widetilde{r}_{j}}{1-q_{j} r_{j}}=d \prod_{j=n}^{+\infty} \frac{1-q_{j} r_{j}}{1-\widetilde{q}_{j} \widetilde{r}_{j}} .
\end{aligned}
$$

The equivalence of the two expressions for each auxiliary variable is related to the fact that the three quantities $\sum_{j=-\infty}^{+\infty} q_{j} r_{j-1}, \sum_{j=-\infty}^{+\infty} r_{j} q_{j-1}$, and $\prod_{j=-\infty}^{+\infty}\left(1-q_{j} r_{j}\right)$ are conserved as in the continuous-time case. These conserved quantities are assumed to be finite, and the last one should be nonzero. The substitution of these expressions into the first two equations in (3.23) provides a global-in-space time discretization of the nonreduced AblowitzLadik lattice (3.19). In the continuum limit of time $h \rightarrow 0$, we obtain

$$
\alpha_{n} \rightarrow \alpha-a q_{n} r_{n-1}, \quad \beta_{n} \rightarrow \beta-b r_{n} q_{n-1}, \quad c_{n} \rightarrow c, \quad d_{n} \rightarrow d .
$$

Thus, with an additional but unessential constraint on the parameters, the time discretization (3.23) reduces to the nonreduced Ablowitz-Ladik lattice (3.19) in the limit $h \rightarrow 0$.

The determinant of the $2 \times 2$ Lax matrix $L_{n}$ (3.20a can be immediately computed as $\operatorname{det} L_{n}=1-q_{n} r_{n}$. We compute the determinant of the $2 \times 2$ 
Lax matrix $V_{n}(3.22)$ as

$$
\begin{aligned}
& \operatorname{det} V_{n}(\lambda)=\lambda^{4} h^{2} a c_{n}+\frac{1}{\lambda^{4}} h^{2} b d_{n} \\
& +\lambda^{2}\left\{h a-h^{2} a^{2} q_{n} \widetilde{r}_{n-1}+h^{2} a \beta_{n}+h^{2} a q_{n-1} \widetilde{r}_{n} d_{n}+h c_{n}\left[1+h \alpha_{n}+h a\left(\widetilde{q}_{n} \widetilde{r}_{n-1}+q_{n} r_{n-1}\right)\right]\right\} \\
& +\frac{1}{\lambda^{2}}\left\{h b-h^{2} b^{2} \widetilde{q}_{n-1} r_{n}+h^{2} b \alpha_{n}+h^{2} b \widetilde{q}_{n} r_{n-1} c_{n}+h d_{n}\left[1+h \beta_{n}+h b\left(\widetilde{q}_{n-1} \widetilde{r}_{n}+q_{n-1} r_{n}\right)\right]\right\} \\
& +h^{2} a b+h^{2} c_{n} d_{n}+\left(1+h \widetilde{q}_{n} r_{n-1} c_{n}+h \alpha_{n}\right)\left(1+h q_{n-1} \widetilde{r}_{n} d_{n}+h \beta_{n}\right) \\
& -h^{2}\left(a q_{n}-\widetilde{q}_{n} c_{n}\right)\left(b r_{n}-\widetilde{r}_{n} d_{n}\right)-h^{2}\left(b \widetilde{q}_{n-1}-q_{n-1} d_{n}\right)\left(a \widetilde{r}_{n-1}-r_{n-1} c_{n}\right) .
\end{aligned}
$$

Thus, the equality (2.10) combined with the boundary conditions (3.24) (or the streamlined version as stated above) implies the set of relations

$$
\left\{\begin{array}{l}
c_{n}=c \Lambda_{n}, \\
d_{n}=d \Lambda_{n} \\
h a-h^{2} a^{2} q_{n} \widetilde{r}_{n-1}+h^{2} a \beta_{n}+h^{2} a q_{n-1} \widetilde{r}_{n} d_{n} \\
+h c_{n}\left[1+h \alpha_{n}+h a\left(\widetilde{q}_{n} \widetilde{r}_{n-1}+q_{n} r_{n-1}\right)\right]=\left[h a+h^{2} a \beta+h c(1+h \alpha)\right] \Lambda_{n}, \\
h b-h^{2} b^{2} \widetilde{q}_{n-1} r_{n}+h^{2} b \alpha_{n}+h^{2} b \widetilde{q}_{n} r_{n-1} c_{n} \\
+h d_{n}\left[1+h \beta_{n}+h b\left(\widetilde{q}_{n-1} \widetilde{r}_{n}+q_{n-1} r_{n}\right)\right]=\left[h b+h^{2} b \alpha+h d(1+h \beta)\right] \Lambda_{n}, \\
h^{2} a b+h^{2} c_{n} d_{n}+\left(1+h \widetilde{q}_{n} r_{n-1} c_{n}+h \alpha_{n}\right)\left(1+h q_{n-1} \widetilde{r}_{n} d_{n}+h \beta_{n}\right) \\
-h^{2}\left(a q_{n}-\widetilde{q}_{n} c_{n}\right)\left(b r_{n}-\widetilde{r}_{n} d_{n}\right)-h^{2}\left(b \widetilde{q}_{n-1}-q_{n-1} d_{n}\right)\left(a \widetilde{r}_{n-1}-r_{n-1} c_{n}\right) \\
=\left[h^{2} a b+h^{2} c d+(1+h \alpha)(1+h \beta)\right] \Lambda_{n} .
\end{array}\right.
$$

Here, $\Lambda_{n}$ is the quantity that satisfies the recurrence formula

$$
\left(1-\widetilde{q}_{n} \widetilde{r}_{n}\right) \Lambda_{n}=\left(1-q_{n} r_{n}\right) \Lambda_{n+1},
$$

and has the normalized boundary value $\lim _{n \rightarrow \pm \infty} \Lambda_{n}=1$. More precisely, only one of the two conditions $\lim _{n \rightarrow-\infty} \Lambda_{n}=1$ and $\lim _{n \rightarrow+\infty} \Lambda_{n}=1$ is required, in accordance with the boundary conditions for $\left(\alpha_{n}, \beta_{n}, c_{n}, d_{n}\right)$. Then, the other condition can be confirmed. Thus, $\Lambda_{n}$ can be written explicitly as

$$
\Lambda_{n}=\prod_{j=-\infty}^{n-1} \frac{1-\widetilde{q}_{j} \widetilde{r}_{j}}{1-q_{j} r_{j}}=\prod_{j=n}^{+\infty} \frac{1-q_{j} r_{j}}{1-\widetilde{q}_{j} \widetilde{r}_{j}}
$$

Note that the first two equalities in (3.25) are valid even if $a=0$ or $b=0$ (cf. (3.23) ). The algebraic system (3.25) essentially comprises three nontrivial relations for the three unknowns: $\alpha_{n}, \beta_{n}$, and $\Lambda_{n}$. Before discussing the general case, we consider two special cases: $a=b=0$ or $c=d=0$. 
- The case $a=b=0$.

In this case, the two auxiliary variables, $\alpha_{n}$ and $\beta_{n}$, become $n$-independent, i.e., $\alpha_{n}=\alpha, \beta_{n}=\beta$. Thus, the discrete-time system (3.23) reduces to

$$
\left\{\begin{array}{l}
\frac{1}{h}\left(\widetilde{q}_{n}-q_{n}\right)+\left(1-q_{n} r_{n}\right) \Lambda_{n+1}\left(c \widetilde{q}_{n+1}-d q_{n-1}\right)-\alpha q_{n}+\beta \widetilde{q}_{n}=0 \\
\frac{1}{h}\left(\widetilde{r}_{n}-r_{n}\right)+\left(1-q_{n} r_{n}\right) \Lambda_{n+1}\left(d \widetilde{r}_{n+1}-c r_{n-1}\right)-\beta r_{n}+\alpha \widetilde{r}_{n}=0 \\
\left(1-\widetilde{q}_{n} \widetilde{r}_{n}\right) \Lambda_{n}=\left(1-q_{n} r_{n}\right) \Lambda_{n+1}, \quad \lim _{n \rightarrow-\infty} \Lambda_{n}=1 \text { or } \lim _{n \rightarrow+\infty} \Lambda_{n}=1,
\end{array}\right.
$$

and the algebraic system (3.25) simplifies to

$$
\begin{aligned}
& h^{2} c d\left(1-\widetilde{q}_{n} \widetilde{r}_{n}\right)\left(1-q_{n-1} r_{n-1}\right) \Lambda_{n}^{2}-\left[(1+h \alpha)(1+h \beta)+h^{2} c d\right. \\
& \left.\quad-(1+h \alpha) h d q_{n-1} \widetilde{r}_{n}-(1+h \beta) h c \widetilde{q}_{n} r_{n-1}\right] \Lambda_{n}+(1+h \alpha)(1+h \beta)=0 .
\end{aligned}
$$

System (3.28) is invariant under the following transformation: $q_{n} \mapsto \mu^{n} q_{n}$, $r_{n} \mapsto \mu^{-n} r_{n}, h c \mapsto \mu^{-1} h c, h d \mapsto \mu h d, h \alpha \mapsto h \alpha, h \beta \mapsto h \beta$, where $\mu$ is a nonzero constant. Using a similar transformation with respect to the time direction, it is possible to remove the parameters $\alpha$ and $\beta$, but we prefer to retain them.

In terms of the normalized parameters

$$
\hat{c}:=\frac{h c}{1+h \alpha}, \quad \hat{d}:=\frac{h d}{1+h \beta},
$$

(3.29) can be rewritten as

$$
\hat{c} \hat{d}\left(1-\widetilde{q}_{n} \widetilde{r}_{n}\right)\left(1-q_{n-1} r_{n-1}\right) \Lambda_{n}^{2}-\left[1+\hat{c} \hat{d}-\hat{d} q_{n-1} \widetilde{r}_{n}-\hat{c} \widetilde{q}_{n} r_{n-1}\right] \Lambda_{n}+1=0 .
$$

When $c d \neq 0$ (and thus $\hat{c} \hat{d} \neq 0$ ), this is a quadratic equation in $\Lambda_{n}$, which has two solutions. The simplest way to reject the improper solution is to recall the asymptotic behavior of the Lax matrix $V_{n}$ for small $h$ (2.8), but here we take a different route. Let us first consider the "trivial" case where $q_{n}$ and $r_{n}$ are zero for all $n$; if this is satisfied at some instant $m=m_{0}$, then it holds true identically for any time $m$. Thus, the two solutions of (3.31) are given by $\Lambda_{n}=1,1 /(\hat{c} \hat{d})$. The recurrence formula for $\Lambda_{n}$ in (3.28) implies that these two solutions are unconnected, i.e., $\Lambda_{n}$ takes the same value for all $n$. The solution $\Lambda_{n}=1 /(\hat{c} \hat{d})$ can be discarded if $\hat{c} \hat{d} \neq 1$, because it is inconsistent with the boundary condition for $\Lambda_{n}$. Next, in the general case 
where $q_{n}$ and $r_{n}$ are not identically zero, we assume that their amplitudes are always so small that their effects can be regarded as weak perturbations of the identically zero case. In particular, the value of $\Lambda_{n}$ is restricted to a neighborhood of unity. Thus, we obtain the proper solution of the quadratic equation (3.31) as

$$
\Lambda_{n}=\frac{2}{1+C_{n}+\sqrt{\left(1+C_{n}\right)^{2}-4 D_{n}}},
$$

with

$$
\begin{aligned}
& C_{n}:=\hat{c} \hat{d}-\hat{d} q_{n-1} \widetilde{r}_{n}-\hat{c} \widetilde{q}_{n} r_{n-1}, \\
& D_{n}:=\hat{c} \hat{d}\left(1-\widetilde{q}_{n} \widetilde{r}_{n}\right)\left(1-q_{n-1} r_{n-1}\right) .
\end{aligned}
$$

Note that this solution is also valid for the linear case $\hat{c} \hat{d}=0$. The decaying boundary conditions for $q_{n}$ and $r_{n}$ imply that $\lim _{n \rightarrow-\infty} \Lambda_{n}=\lim _{n \rightarrow+\infty} \Lambda_{n}=1$. When $\hat{c} \hat{d} \in \mathbb{R}$, the local expression (3.32) is valid only if $\hat{c} \hat{d} \leq 1$. If $\hat{c} \hat{d}>1$, the other solution of (3.31) should be adopted; alternatively, one can understand the right-hand side of (3.32) as being defined by a Taylor series for small $\hat{c}$ and $\hat{d}$, and its analytic continuation. At the "threshold" value $\hat{c} \hat{d}=1$, the discrete-time system (3.28) has the trivial solution $\widetilde{q}_{n}=\hat{d} q_{n-1}$, $\widetilde{r}_{n}=\hat{c} r_{n-1}, \Lambda_{n}=1 /\left(1-q_{n-1} r_{n-1}\right)$. Thus, the discriminant of the quadratic equation (3.31) vanishes at $\hat{c} \hat{d}=1$, and the two solutions indeed intersect. Unless $\hat{c} \hat{d}=1$, a unified expression for $\Lambda_{n}$,

$$
\Lambda_{n}=\frac{2}{1+C_{n}+(1-\hat{c} \hat{d}) \sqrt{\left(\frac{1+C_{n}}{1-\hat{c} \hat{d}}\right)^{2}-\frac{4 D_{n}}{(1-\hat{c} \hat{d})^{2}}}}
$$

can resolve the sign problem of the square root. In addition, the above square root allows the Taylor expansion with respect to $\left\{\widetilde{q}_{n}, \widetilde{r}_{n}, q_{n-1}, r_{n-1}\right\}$. However, we do not use this unwieldy formula.

The first and second equations in (3.28) are linear in $\widetilde{q}_{n}$ and $\widetilde{r}_{n}$, respectively. Thus, the forward time evolution can be expressed as follows (cf. (3.30) ):

$$
\left\{\begin{array}{l}
\widetilde{q}_{n}=\frac{1+h \alpha}{1+h \beta} q_{n}+\left(1-q_{n} r_{n}\right) \Lambda_{n+1}\left(-\frac{1+h \alpha}{1+h \beta} \hat{c} \widetilde{q}_{n+1}+\hat{d} q_{n-1}\right), \\
\widetilde{r}_{n}=\frac{1+h \beta}{1+h \alpha} r_{n}+\left(1-q_{n} r_{n}\right) \Lambda_{n+1}\left(-\frac{1+h \beta}{1+h \alpha} \hat{d} \widetilde{r}_{n+1}+\hat{c} r_{n-1}\right),
\end{array}\right.
$$

where $\Lambda_{n}$ is given by (3.32) with (3.33). In the simplest case of $c=\hat{c}=0$ or $d=\hat{d}=0$, the forward time evolution (3.34) is given by a simple rational 
mapping. This fact was disclosed by Suris [7, 19]. In the general case, using the new parameters $\check{c}:=\hat{c}(1+\hat{c} \hat{d})^{-1}$ and $\check{d}:=\hat{d}(1+\hat{c} \hat{d})^{-1}$, the mapping (3.34) can be rewritten in a slightly simpler form:

$$
\left\{\begin{array}{l}
\widetilde{q}_{n}=\frac{1+h \alpha}{1+h \beta} q_{n}+\frac{2\left(1-q_{n} r_{n}\right)}{F_{n}}\left(-\frac{1+h \alpha}{1+h \beta} \check{c} \widetilde{q}_{n+1}+\check{d} q_{n-1}\right), \\
\widetilde{r}_{n}=\frac{1+h \beta}{1+h \alpha} r_{n}+\frac{2\left(1-q_{n} r_{n}\right)}{F_{n}}\left(-\frac{1+h \beta}{1+h \alpha} \check{d} \widetilde{r}_{n+1}+\check{c} r_{n-1}\right),
\end{array}\right.
$$

with

$$
\begin{aligned}
F_{n}:= & 1-\check{d} q_{n} \widetilde{r}_{n+1}-\check{c} \widetilde{q}_{n+1} r_{n} \\
& +\sqrt{\left(1-\check{d} q_{n} \widetilde{r}_{n+1}-\check{c} \widetilde{q}_{n+1} r_{n}\right)^{2}-4 \check{c} \check{d}\left(1-\widetilde{q}_{n+1} \widetilde{r}_{n+1}\right)\left(1-q_{n} r_{n}\right)} .
\end{aligned}
$$

That is, the first term $\hat{c} \hat{d}$ of $C_{n+1}$ is removed. When $\hat{c} \hat{d} \in \mathbb{R}$, this expression for $F_{n}$ is valid only if $-1<\hat{c} \hat{d} \leq 1$; this corresponds to the range $\check{c} \breve{d} \leq 1 / 4$. If $\hat{c} \hat{d}<-1$ or $\hat{c} \hat{d}>1$, the sign in front of the square root has to be changed.

When $h$ is real, $\beta=\alpha^{*}$, and $d=c^{*}$, we can impose the complex conjugacy reduction $r_{n}=\sigma q_{n}^{*}$ with a real constant $\sigma$. In particular, setting $\alpha=-\beta=-\mathrm{i} \gamma / \Delta^{2}, c=-d=-\mathrm{i} / \Delta^{2}$, and $r_{n}=-\Delta^{2} q_{n}^{*}$, we obtain the fully discretized NLS equation

$$
\frac{\mathrm{i}}{h}\left(\widetilde{q}_{n}-q_{n}\right)+\frac{2\left(1+\Delta^{2}\left|q_{n}\right|^{2}\right)}{1+C_{n+1}+\sqrt{\left(1+C_{n+1}\right)^{2}-4 D_{n+1}}} \frac{\widetilde{q}_{n+1}+q_{n-1}}{\Delta^{2}}-\frac{\gamma}{\Delta^{2}}\left(\widetilde{q}_{n}+q_{n}\right)=0,
$$

where

$$
\begin{aligned}
C_{n+1} & =\frac{h^{2}}{\left(\Delta^{2}+\mathrm{i} \gamma h\right)\left(\Delta^{2}-\mathrm{i} \gamma h\right)}+\frac{\mathrm{i} \Delta^{2} h}{\Delta^{2}+\mathrm{i} \gamma h} q_{n} \widetilde{q}_{n+1}^{*}-\frac{\mathrm{i} \Delta^{2} h}{\Delta^{2}-\mathrm{i} \gamma h} \widetilde{q}_{n+1} q_{n}^{*}, \\
D_{n+1} & =\frac{h^{2}}{\left(\Delta^{2}+\mathrm{i} \gamma h\right)\left(\Delta^{2}-\mathrm{i} \gamma h\right)}\left(1+\Delta^{2}\left|\widetilde{q}_{n+1}\right|^{2}\right)\left(1+\Delta^{2}\left|q_{n}\right|^{2}\right) .
\end{aligned}
$$

The choice of $\gamma=1$ for the real parameter $\gamma$ is the most natural when taking the continuum limit, while the choice of $\gamma=0$ simplifies the equation considerably. The aforementioned condition $\hat{c} \hat{d} \leq 1$ implies that $\left(1-\gamma^{2}\right) h^{2} \leq \Delta^{4}$; this is automatically satisfied if $\gamma^{2} \geq 1$. At first glance, it is far from evident that the quantity in the square root is nonnegative: $\left(1+C_{n+1}\right)^{2}-4 D_{n+1} \geq 0$. However, this inequality should hold true because the left-hand side represents the discriminant of the quadratic equation in $\Lambda_{n+1}$ (cf. (3.31) ). In fact, 
the reduction $r_{n}=-\Delta^{2} q_{n}^{*}$ with $\Delta^{2}>0$ guarantees the auxiliary variable $\Lambda_{n}$ to be positive (cf. (3.27)). Thus, we also have the inequality $1+C_{n+1}>0$. To summarize, $\widetilde{q}_{n+1}$ and $q_{n}$ are not fully independent and satisfy the inequality $1+C_{n+1} \geq 2 \sqrt{D_{n+1}}$. Note that (3.35) with $n \rightarrow n+1$ determines $\widetilde{q}_{n+1}$ from $q_{n+1}, \widetilde{q}_{n+2}$, and $q_{n}$.

Similarly to the continuous NLS equation, this full discretization is homogeneous under the following weighting scheme: weight $(\Delta)=-1$, weight $(h)=-2$, weight $\left(q_{n}\right)=1$. At present, it is unclear whether the corresponding oneparameter group of scaling symmetries, $\left(\Delta, h, q_{n}\right) \mapsto\left(\Delta / k, h / k^{2}, k q_{n}\right)$, can define meaningful "self-similar" solutions to (3.35).

As mentioned previously, it might be aesthetically pleasing to eliminate the first "constant" term of $C_{n+1}$ by a suitable redefinition of the parameters. For example, in the simple case of $\gamma=0$ and $\Delta^{2}=1$, (3.35) can be rewritten as

$$
\frac{\mathrm{i}}{\delta}\left(\widetilde{q}_{n}-q_{n}\right)+\frac{2\left(1+\left|q_{n}\right|^{2}\right)\left(\widetilde{q}_{n+1}+q_{n-1}\right)}{1+\mathrm{i} \delta \mathcal{C}_{n}+\sqrt{\left(1+\mathrm{i} \delta \mathcal{C}_{n}\right)^{2}-4 \delta^{2} \mathcal{D}_{n}}}=0
$$

with

$$
\mathcal{C}_{n}:=q_{n} \widetilde{q}_{n+1}^{*}-\widetilde{q}_{n+1} q_{n}^{*}, \quad \mathcal{D}_{n}:=\left(1+\left|\widetilde{q}_{n+1}\right|^{2}\right)\left(1+\left|q_{n}\right|^{2}\right) .
$$

Here, $\delta:=h /\left(1+h^{2}\right)$ is a new parameter. Because $-1 \leq h \leq 1$, the range of $\delta$ is $-1 / 2 \leq \delta \leq 1 / 2$; at the end points $\delta= \pm 1 / 2$, the time evolution is trivial because $\hat{c} \hat{d}=1$. Note that (3.36) is invariant under the transformation $q_{n} \mapsto(-1)^{n} q_{n}, \delta \mapsto-\delta$.

To express the backward time evolution explicitly, we only have to replace $\left(1-q_{n} r_{n}\right) \Lambda_{n+1}$ in the first and second equalities of (3.28) with $\left(1-\widetilde{q}_{n} \widetilde{r}_{n}\right) \Lambda_{n}$, and then substitute the local expression (3.32) with (3.33).

In the complex conjugacy reduction wherein $h$ is real, $\beta=\alpha^{*}$, and $d=c^{*}$, we can "normalize" the scaling of the dependent variable by setting $r_{n}=-q_{n}^{*}$. In this case, we rewrite the first equation for $q_{n}$ in (3.28) as

$$
\left(1+h \alpha^{*}\right) \widetilde{q}_{n}-(1+h \alpha) q_{n}+\left(1+\left|\widetilde{q}_{n}\right|^{2}\right) \Lambda_{n}\left(h c \widetilde{q}_{n+1}-h c^{*} q_{n-1}\right)=0 .
$$

Moreover, (3.29) can be rewritten as

$$
\begin{aligned}
& |1+h \alpha|^{2}+h^{2}|c|^{2}+(1+h \alpha) h c^{*} q_{n-1} \widetilde{q}_{n}^{*}+\left(1+h \alpha^{*}\right) h c \widetilde{q}_{n} q_{n-1}^{*} \\
& =|1+h \alpha|^{2} \frac{1}{\Lambda_{n}}+h^{2}|c|^{2}\left(1+\left|\widetilde{q}_{n}\right|^{2}\right)\left(1+\left|q_{n-1}\right|^{2}\right) \Lambda_{n} .
\end{aligned}
$$

Using (3.37), we can replace $1 / \Lambda_{n}$ and $\Lambda_{n}$ in (3.38) with rational expressions in $q_{n}$ as well as its shifts and complex conjugate. Thus, we obtain a rational 
form of the fully discrete NLS equation,

$$
\begin{aligned}
& |1+h \alpha|^{2}+h^{2}|c|^{2}+2 \operatorname{Re}\left[(1+h \alpha) h c^{*} q_{n-1} \widetilde{q}_{n}^{*}\right] \\
& =|1+h \alpha|^{2}\left(1+\left|\widetilde{q}_{n}\right|^{2}\right) \frac{h c \widetilde{q}_{n+1}-h c^{*} q_{n-1}}{(1+h \alpha) q_{n}-\left(1+h \alpha^{*}\right) \widetilde{q}_{n}} \\
& \quad+h^{2}|c|^{2}\left(1+\left|q_{n-1}\right|^{2}\right) \frac{(1+h \alpha) q_{n}-\left(1+h \alpha^{*}\right) \widetilde{q}_{n}}{h c \widetilde{q}_{n+1}-h c^{*} q_{n-1}} .
\end{aligned}
$$

Surprisingly, this coincides with the double-discrete NLS equation proposed by Quispel, Nijhoff, Capel, and van der Linden [15, 17, up to a minor change of coordinates and parameters; despite its "elegance", this rational version has the drawback that the forward/backward time evolution cannot be uniquely determined. In addition, one cannot immediately recognize that (3.39) reduces to the NLS equation in a continuous limit. Thus, we prefer our version, which is seemingly less elegant because of the existence of the square root but can define the unique time evolution properly and allow an easy-to-follow continuous limit.

Actually, using a coordinate transformation, it is possible to "identify" our time discretization [(3.34) with $\hat{d}=\hat{c}^{*}, \alpha=\beta=0$, and $r_{n}=-q_{n}^{*}$ ] with the auto-Bäcklund transformation of the Ablowitz-Ladik hierarchy derived by Nijhoff, Quispel, and Capel [14]. However, their expression (see (19) in [14]) involves the indefinite sign \pm in front of the square root and it is not clear how to understand and determine it.

Once the local expressions for the auxiliary variables have been derived, we can normalize the $2 \times 2$ matrix Lax pair, $L_{n}$ and $V_{n}$, so that $\operatorname{det} L_{n}$ and $\operatorname{det} V_{n}$ become equal to 1 . Indeed, this is easily achieved by dividing $L_{n}$ and $V_{n}$ by $\sqrt{\operatorname{det} L_{n}}$ and $\sqrt{\operatorname{det} V_{n}}$, respectively (cf. (2.6) and (2.10)). However, the normalized Lax pair not involving the auxiliary variables appears to be rather cumbersome and we do not present it here.

- The case $c=d=0$.

In this case, the two auxiliary variables $c_{n}$ and $d_{n}$ vanish. Thus, the discrete-time system (3.23) reduces to

$$
\left\{\begin{array}{l}
\frac{1}{h}\left(\widetilde{q}_{n}-q_{n}\right)-a q_{n+1}+b \widetilde{q}_{n-1}-a\left(\widetilde{q}_{n} \widetilde{r}_{n-1}-q_{n+1} r_{n}\right) q_{n}-\alpha_{n} q_{n}+\widetilde{q}_{n} \beta_{n}=0, \\
\frac{1}{h}\left(\widetilde{r}_{n}-r_{n}\right)-b r_{n+1}+a \widetilde{r}_{n-1}-b\left(\widetilde{r}_{n} \widetilde{q}_{n-1}-r_{n+1} q_{n}\right) r_{n}-\beta_{n} r_{n}+\widetilde{r}_{n} \alpha_{n}=0, \\
\alpha_{n+1}-\alpha_{n}=a\left(\widetilde{q}_{n} \widetilde{r}_{n-1}-q_{n+1} r_{n}\right), \\
\beta_{n+1}-\beta_{n}=b\left(\widetilde{r}_{n} \widetilde{q}_{n-1}-r_{n+1} q_{n}\right),
\end{array}\right.
$$


where $\lim _{n \rightarrow-\infty}\left(\alpha_{n}, \beta_{n}\right)=(\alpha, \beta)$ or $\lim _{n \rightarrow+\infty}\left(\alpha_{n}, \beta_{n}\right)=(\alpha, \beta)$. The algebraic system (3.25) simplifies to

$$
\left\{\begin{array}{l}
1-h a q_{n} \widetilde{r}_{n-1}+h \beta_{n}=(1+h \beta) \Lambda_{n} \text { if } a \neq 0, \\
1-h b \widetilde{q}_{n-1} r_{n}+h \alpha_{n}=(1+h \alpha) \Lambda_{n} \text { if } b \neq 0, \\
h^{2} a b\left(1-q_{n} r_{n}-\widetilde{q}_{n-1} \widetilde{r}_{n-1}\right)+\left(1+h \alpha_{n}\right)\left(1+h \beta_{n}\right) \\
=\left[h^{2} a b+(1+h \alpha)(1+h \beta)\right] \Lambda_{n} .
\end{array}\right.
$$

System (3.40) is invariant under the following transformation: $q_{n} \mapsto \mu^{n} q_{n}$, $r_{n} \mapsto \mu^{-n} r_{n}, h a \mapsto \mu^{-1} h a, h b \mapsto \mu h b, h \alpha \mapsto h \alpha, h \beta \mapsto h \beta$, where $\mu$ is a nonzero constant.

It can be easily verified that the first equality in (3.41) is also valid for $a=0$, and the second equality is valid for $b=0$. Using the first and second equalities, the third equality in (3.41) results in a quadratic equation in $\Lambda_{n}$,

$$
\begin{aligned}
& (1+h \alpha)(1+h \beta) \Lambda_{n}^{2}-\left[(1+h \alpha)(1+h \beta)+h^{2} a b-(1+h \alpha) h a q_{n} \widetilde{r}_{n-1}\right. \\
& \left.-(1+h \beta) h b \widetilde{q}_{n-1} r_{n}\right] \Lambda_{n}+h^{2} a b\left(1-q_{n} r_{n}\right)\left(1-\widetilde{q}_{n-1} \widetilde{r}_{n-1}\right)=0 .
\end{aligned}
$$

In terms of the normalized parameters

$$
\hat{a}:=\frac{h a}{1+h \beta}, \quad \hat{b}:=\frac{h b}{1+h \alpha},
$$

(3.42) can be rewritten as

$$
\Lambda_{n}^{2}-\left(1+\hat{a} \hat{b}-\hat{a} q_{n} \widetilde{r}_{n-1}-\hat{b} \widetilde{q}_{n-1} r_{n}\right) \Lambda_{n}+\hat{a} \hat{b}\left(1-q_{n} r_{n}\right)\left(1-\widetilde{q}_{n-1} \widetilde{r}_{n-1}\right)=0 .
$$

We recall that the Lax matrix $V_{n}$ given by (3.22) with $c_{n}=d_{n}=0$ is required to allow the asymptotic expansion (2.8) for small $h$. This implies that the auxiliary variables $\alpha_{n}$ and $\beta_{n}$ determined by (3.41) are at most of order $O(1)$ and do not involve $1 / h$. Thus, one of the two solutions of the quadratic equation (3.44) is rejected, and we obtain its proper solution as

$$
\Lambda_{n}=\frac{1+A_{n}+\sqrt{\left(1+A_{n}\right)^{2}-4 B_{n}}}{2},
$$

with

$$
\begin{aligned}
& A_{n}:=\hat{a} \hat{b}-\hat{a} q_{n} \widetilde{r}_{n-1}-\hat{b} \widetilde{q}_{n-1} r_{n}, \\
& B_{n}:=\hat{a} \hat{b}\left(1-q_{n} r_{n}\right)\left(1-\widetilde{q}_{n-1} \widetilde{r}_{n-1}\right) .
\end{aligned}
$$


The decaying boundary conditions for $q_{n}$ and $r_{n}$ imply that $\lim _{n \rightarrow-\infty} \Lambda_{n}=\lim _{n \rightarrow+\infty} \Lambda_{n}=1$, and consequently, $\lim _{n \rightarrow-\infty}\left(\alpha_{n}, \beta_{n}\right)=\lim _{n \rightarrow+\infty}\left(\alpha_{n}, \beta_{n}\right)=(\alpha, \beta)$. When $\hat{a} \hat{b} \in \mathbb{R}$, the local expression (3.45) is valid only if $\hat{a} \hat{b} \leq 1$. If $\hat{a} \hat{b}>1$, the other solution of (3.44) should be employed; alternatively, one can understand the right-hand side of (3.45) as being defined by a Taylor series for small $\hat{a}$ and $\hat{b}$, and its analytic continuation. At the "threshold" value $\hat{a} \hat{b}=1$, the discretetime system (3.40) has the trivial solution $\widetilde{q}_{n}=\hat{a} q_{n+1}, \widetilde{r}_{n}=\hat{b} r_{n+1}, \alpha_{n}=\alpha$, $\beta_{n}=\beta$. Thus, the discriminant of the quadratic equation (3.44) vanishes at $\hat{a} \hat{b}=1$, and the two solutions indeed intersect. Unless $\hat{a} \hat{b}=1$, a unified expression for $\Lambda_{n}$,

$$
\Lambda_{n}=\frac{1+A_{n}+(1-\hat{a} \hat{b}) \sqrt{\left(\frac{1+A_{n}}{1-\hat{a} \hat{b}}\right)^{2}-\frac{4 B_{n}}{(1-\hat{a} \hat{b})^{2}}}}{2}
$$

can resolve the sign problem of the square root. In addition, this expression allows the Taylor expansion with respect to $\left\{q_{n}, r_{n}, \widetilde{q}_{n-1}, \widetilde{r}_{n-1}\right\}$. However, we avoid the use of this unwieldy form.

Using the first two relations in (3.41), we can rewrite $\alpha_{n}$ and $\beta_{n}$ in the first two relations in (3.40) in terms of $\Lambda_{n}$,

$$
\left\{\begin{array}{l}
\frac{1}{h}\left(\widetilde{q}_{n}-q_{n}\right)+\beta \widetilde{q}_{n}-\alpha q_{n}+\frac{1-q_{n} r_{n}}{\Lambda_{n}}\left(-a q_{n+1}+b \widetilde{q}_{n-1}\right)=0, \\
\frac{1}{h}\left(\widetilde{r}_{n}-r_{n}\right)+\alpha \widetilde{r}_{n}-\beta r_{n}+\frac{1-q_{n} r_{n}}{\Lambda_{n}}\left(-b r_{n+1}+a \widetilde{r}_{n-1}\right)=0 .
\end{array}\right.
$$

This system is linear in $\widetilde{q}_{n}$ and $\widetilde{r}_{n}$. Thus, the forward time evolution can be expressed as follows (cf. (3.43)):

$$
\left\{\begin{array}{l}
\widetilde{q}_{n}=\frac{1+h \alpha}{1+h \beta} q_{n}+\frac{1-q_{n} r_{n}}{\Lambda_{n}}\left(\hat{a} q_{n+1}-\frac{1+h \alpha}{1+h \beta} \hat{b} \widetilde{q}_{n-1}\right) \\
\widetilde{r}_{n}=\frac{1+h \beta}{1+h \alpha} r_{n}+\frac{1-q_{n} r_{n}}{\Lambda_{n}}\left(\hat{b} r_{n+1}-\frac{1+h \beta}{1+h \alpha} \hat{a} \widetilde{r}_{n-1}\right)
\end{array}\right.
$$

where $\Lambda_{n}$ is given by (3.45) with (3.46).

In the simplest case of $a=\hat{a}=0$ or $b=\hat{b}=0$, the quadratic equation (3.44) in $\Lambda_{n}$ reduces to a linear equation, and the forward time evolution (3.48) is given by a simple rational mapping. This fact was discovered by Suris [7, 19]; actually, slightly prior to his work, relevant results in a preliminary form were reported by Pempinelli, Boiti, and Leon [40].

When $h$ is real, $\beta=\alpha^{*}$, and $b=a^{*}$, we can impose the complex conjugacy reduction $r_{n}=\sigma q_{n}^{*}$ with a real constant $\sigma$. In particular, setting 
$\alpha=-\beta=-\mathrm{i} \gamma / \Delta^{2}, a=-b=\mathrm{i} / \Delta^{2}$, and $r_{n}=-\Delta^{2} q_{n}^{*}$, we obtain the fully discretized NLS equation

$$
\frac{\mathrm{i}}{h}\left(\widetilde{q}_{n}-q_{n}\right)+\frac{2\left(1+\Delta^{2}\left|q_{n}\right|^{2}\right)}{1+A_{n}+\sqrt{\left(1+A_{n}\right)^{2}-4 B_{n}}} \frac{q_{n+1}+\widetilde{q}_{n-1}}{\Delta^{2}}-\frac{\gamma}{\Delta^{2}}\left(\widetilde{q}_{n}+q_{n}\right)=0
$$

where

$$
\begin{aligned}
& A_{n}=\frac{h^{2}}{\left(\Delta^{2}+\mathrm{i} \gamma h\right)\left(\Delta^{2}-\mathrm{i} \gamma h\right)}+\frac{\mathrm{i} \Delta^{2} h}{\Delta^{2}+\mathrm{i} \gamma h} q_{n} \widetilde{q}_{n-1}^{*}-\frac{\mathrm{i} \Delta^{2} h}{\Delta^{2}-\mathrm{i} \gamma h} \widetilde{q}_{n-1} q_{n}^{*}, \\
& B_{n}=\frac{h^{2}}{\left(\Delta^{2}+\mathrm{i} \gamma h\right)\left(\Delta^{2}-\mathrm{i} \gamma h\right)}\left(1+\Delta^{2}\left|q_{n}\right|^{2}\right)\left(1+\Delta^{2}\left|\widetilde{q}_{n-1}\right|^{2}\right) .
\end{aligned}
$$

Thus, this is equivalent to the fully discrete NLS equation (3.35) in the case $a=b=0$, up to the space reflection $n \rightarrow-n$; this correspondence can be readily noticed by considering the dispersion relation [3]. The choice of $\gamma=1$ for the real parameter $\gamma$ is the most natural for taking the continuum limit, while the choice of $\gamma=0$ simplifies the equation considerably. The aforementioned condition $\hat{a} \hat{b} \leq 1$ implies that $\left(1-\gamma^{2}\right) h^{2} \leq \Delta^{4}$; this is automatically satisfied if $\gamma^{2} \geq 1$. Note that $\widetilde{q}_{n-1}$ and $q_{n}$ are correlated and always satisfy the inequality $1+A_{n} \geq 2 \sqrt{B_{n}}$ if $\Delta^{2}>0$.

To express the backward time evolution explicitly, we have to rewrite $\Lambda_{n}$ in (3.47) in terms of $\Lambda_{n+1}$ using (3.26), and then substitute the local expression (3.45) with (3.46). By this procedure, the fully discrete NLS equation (3.49) in the case $c=d=0$ can also be identified with the fully discrete NLS equation (3.35) in the case $a=b=0$ through the time reflection $m \rightarrow-m$ and the sign inversion of $h, h \rightarrow-h$ [3, 7,,19].

Note that the auxiliary variables appearing in the stage of time discretization can be interpreted, after a minor redefinition, as giving the fluxes of the first few conservation laws. Indeed, (3.26) and (3.40) provide the following conservation laws:

$$
\begin{aligned}
& \boldsymbol{\Delta}_{m}^{+} \log \left(1-q_{n} r_{n}\right)=\boldsymbol{\Delta}_{n}^{+} \log \Lambda_{n}, \\
& \boldsymbol{\Delta}_{m}^{+}\left(q_{n} r_{n-1}\right)=\boldsymbol{\Delta}_{n}^{+}\left[\left(\alpha_{n}-\alpha\right) / a+q_{n} r_{n-1}\right], \\
& \boldsymbol{\Delta}_{m}^{+}\left(q_{n-1} r_{n}\right)=\boldsymbol{\Delta}_{n}^{+}\left[\left(\beta_{n}-\beta\right) / b+q_{n-1} r_{n}\right] .
\end{aligned}
$$

The cases $a=0$ and $b=0$ can be understood as limiting cases; the final local expressions for the fluxes do not contain $1 / a$ and $1 / b$, and also hold true for these cases. Using (3.26), (3.41), (3.43), (3.45), and (3.48), we can express 
the fluxes in (3.50b) and (3.50c) explicitly as

$$
\begin{aligned}
& \frac{1}{a}\left(\alpha_{n}-\alpha\right)+q_{n} r_{n-1}=\frac{1+h \alpha}{h a}\left[\Lambda_{n}-1+\hat{b} \widetilde{q}_{n-1} r_{n}\right]+q_{n} r_{n-1} \\
& =\frac{1+h \alpha}{h a}\left[-\frac{1+A_{n}-\sqrt{\left(1+A_{n}\right)^{2}-4 B_{n}}}{2}+\hat{a} \hat{b}-\hat{a} q_{n} \widetilde{r}_{n-1}\right]+q_{n} r_{n-1} \\
& =\frac{1+h \alpha}{1+h \beta}\left[-\frac{2 \hat{b}\left(1-q_{n} r_{n}\right)\left(1-\widetilde{q}_{n-1} \widetilde{r}_{n-1}\right)}{1+A_{n}+\sqrt{\left(1+A_{n}\right)^{2}-4 B_{n}}}+\hat{b}-q_{n} \widetilde{r}_{n-1}\right]+q_{n} r_{n-1} \\
& =\frac{h}{1+h \beta}\left[\frac{2\left(1-\widetilde{q}_{n-1} \widetilde{r}_{n-1}\right)\left(-b+a q_{n} \widetilde{r}_{n-2}\right)}{1+A_{n}+\sqrt{\left(1+A_{n}\right)^{2}-4 B_{n}}}+b\right] \text {, } \\
& \frac{1}{b}\left(\beta_{n}-\beta\right)+q_{n-1} r_{n}=\frac{1+h \beta}{h b}\left[\Lambda_{n}-1+\hat{a} q_{n} \widetilde{r}_{n-1}\right]+q_{n-1} r_{n} \\
& =\frac{1+h \beta}{h b}\left[-\frac{1+A_{n}-\sqrt{\left(1+A_{n}\right)^{2}-4 B_{n}}}{2}+\hat{a} \hat{b}-\hat{b} \widetilde{q}_{n-1} r_{n}\right]+q_{n-1} r_{n} \\
& =\frac{1+h \beta}{1+h \alpha}\left[-\frac{2 \hat{a}\left(1-q_{n} r_{n}\right)\left(1-\widetilde{q}_{n-1} \widetilde{r}_{n-1}\right)}{1+A_{n}+\sqrt{\left(1+A_{n}\right)^{2}-4 B_{n}}}+\hat{a}-\widetilde{q}_{n-1} r_{n}\right]+q_{n-1} r_{n} \\
& =\frac{h}{1+h \alpha}\left[\frac{2\left(1-\widetilde{q}_{n-1} \widetilde{r}_{n-1}\right)\left(-a+b \widetilde{q}_{n-2} r_{n}\right)}{1+A_{n}+\sqrt{\left(1+A_{n}\right)^{2}-4 B_{n}}}+a\right] \text {, }
\end{aligned}
$$

where $A_{n}$ and $B_{n}$ are given by (3.46). Note that using (3.26) and (3.48), we can further rewrite these fluxes in many different forms.

- The general case $(a, b) \neq \mathbf{0}$ and $(c, d) \neq \mathbf{0}$.

Let us consider the (nonreduced) discrete-time Ablowitz-Ladik lattice (3.23) with (3.24) in the general case of $(a, b) \neq \mathbf{0}$ and $(c, d) \neq \mathbf{0}$. Before dealing with the general case of the parameters $a, b, c, d, \alpha$, and $\beta$, we first consider the special case where the conditions $c=-a(\neq 0), d=-b(\neq 0)$, and $\beta=-\alpha$ are satisfied. This is a particularly interesting case from the perspective of applications such as numerical experiments, because the linear part of the equations for $q_{n}$ and $r_{n}$ has symmetry with respect to the time reflection $m \rightarrow-m$ combined with the sign inversion of $h$. Moreover, the symmetry with respect to the space reflection $n \rightarrow-n$ can also be achieved by imposing the further condition $b=-a[1,3,5,19,41$. 
In the case of $c=-a(\neq 0), d=-b(\neq 0)$, and $\beta=-\alpha$, the algebraic system (3.25) can be reformulated as

$$
\left\{\begin{array}{l}
\left(1-h b q_{n-1} \widetilde{r}_{n} \Lambda_{n}+h \beta_{n}\right)-\Lambda_{n}\left(1-h a \widetilde{q}_{n} r_{n-1} \Lambda_{n}+h \alpha_{n}\right) \\
=h a q_{n} \widetilde{r}_{n-1}+\left[-2 h \alpha+h a\left(\widetilde{q}_{n} \widetilde{r}_{n-1}+q_{n} r_{n-1}\right)\right] \Lambda_{n}+h a \widetilde{q}_{n} r_{n-1} \Lambda_{n}^{2}, \\
\left(1-h a \widetilde{q}_{n} r_{n-1} \Lambda_{n}+h \alpha_{n}\right)-\Lambda_{n}\left(1-h b q_{n-1} \widetilde{r}_{n} \Lambda_{n}+h \beta_{n}\right) \\
=h b \widetilde{q}_{n-1} r_{n}+\left[2 h \alpha+h b\left(\widetilde{q}_{n-1} \widetilde{r}_{n}+q_{n-1} r_{n}\right)\right] \Lambda_{n}+h b q_{n-1} \widetilde{r}_{n} \Lambda_{n}^{2} \\
\left(1-h a \widetilde{q}_{n} r_{n-1} \Lambda_{n}+h \alpha_{n}\right)\left(1-h b q_{n-1} \widetilde{r}_{n} \Lambda_{n}+h \beta_{n}\right) \\
=-h^{2} a b\left(1-q_{n} r_{n}-\widetilde{q}_{n-1} \widetilde{r}_{n-1}\right)-h^{2} a b\left(1-\widetilde{q}_{n} \widetilde{r}_{n}-q_{n-1} r_{n-1}\right) \Lambda_{n}^{2} \\
\quad+\left[h^{2} a b\left(2+q_{n} \widetilde{r}_{n}+\widetilde{q}_{n} r_{n}+\widetilde{q}_{n-1} r_{n-1}+q_{n-1} \widetilde{r}_{n-1}\right)+(1+h \alpha)(1-h \alpha)\right] \Lambda_{n},
\end{array}\right.
$$

with $c_{n}=-a \Lambda_{n}$ and $d_{n}=-b \Lambda_{n}$. Using the first two relations, we can express $1-h a \widetilde{q}_{n} r_{n-1} \Lambda_{n}+h \alpha_{n}$ and $1-h b q_{n-1} \widetilde{r}_{n} \Lambda_{n}+h \beta_{n}$ in terms of $\Lambda_{n}$. Substituting these expressions into the third relation, we obtain a sixth-degree equation in only $\Lambda_{n}$. However, this sextic equation turns out to be too complicated for exact treatment. Thus, we need to take a different route. One sensible approach is to solve the algebraic system (3.51) approximately; for example, using perturbative expansions with respect to $h$, we obtain approximate expressions for the auxiliary variables as follows:

$$
\left\{\begin{array}{l}
\alpha_{n}-a \widetilde{q}_{n} r_{n-1} \Lambda_{n}=\alpha-\frac{a}{2}\left(\widetilde{q}_{n}+q_{n}\right)\left(\widetilde{r}_{n-1}+r_{n-1}\right)+O(h), \\
\beta_{n}-b q_{n-1} \widetilde{r}_{n} \Lambda_{n}=-\alpha-\frac{b}{2}\left(\widetilde{q}_{n-1}+q_{n-1}\right)\left(\widetilde{r}_{n}+r_{n}\right)+O(h), \\
\Lambda_{n}=1-\frac{h a}{2}\left(\widetilde{q}_{n}+q_{n}\right)\left(\widetilde{r}_{n-1}+r_{n-1}\right)-\frac{h b}{2}\left(\widetilde{q}_{n-1}+q_{n-1}\right)\left(\widetilde{r}_{n}+r_{n}\right)+O\left(h^{2}\right) .
\end{array}\right.
$$

Combining (3.52) with (3.23) for $c=-a, d=-b$, and $\beta=-\alpha$, we obtain a high-precision numerical scheme that is "almost integrable" (cf. [3, 5, 41]). We can also consider the next order in $h$ and include higher-order corrections.

We now discuss the general case where no particular conditions on the parameters $a, b, c, d, \alpha$, and $\beta$ are assumed. Instead of exploiting the complicated algebraic system (3.25), we explain how to recreate the discrete-time Ablowitz-Ladik lattice (3.23) by the composition of a few more elementary time evolutions such as those studied earlier for the special cases $a=b=0$ or $c=d=0$. To this end, we recall some basic properties of integrable hierarchies. That is, under suitable boundary conditions, an integrable hierarchy generally comprises infinitely many commuting flows. Any composition of these flows also belongs to the same hierarchy; each of the flows is uniquely determined by specifying its linear part, or equivalently, its dispersion relation. Under the boundary conditions (3.24) or, equivalently, the streamlined 
version, the discrete-time Ablowitz-Ladik lattice (3.23) has the following linear leading part:

$$
\left\{\begin{array}{l}
\frac{1}{h}\left(\widetilde{q}_{n}-q_{n}\right)+c \widetilde{q}_{n+1}+\beta \widetilde{q}_{n}+b \widetilde{q}_{n-1}-a q_{n+1}-\alpha q_{n}-d q_{n-1} \sim 0, \\
\frac{1}{h}\left(\widetilde{r}_{n}-r_{n}\right)+d \widetilde{r}_{n+1}+\alpha \widetilde{r}_{n}+a \widetilde{r}_{n-1}-b r_{n+1}-\beta r_{n}-c r_{n-1} \sim 0 .
\end{array}\right.
$$

Substituting the ansatz $q_{n} \sim \lambda^{2 n}, \widetilde{q}_{n} \sim \lambda^{2 n} \omega, r_{n} \sim \lambda^{-2 n}, \widetilde{r}_{n} \sim \lambda^{-2 n} \omega^{-1}$ into (3.53), we obtain the corresponding dispersion relation [3]

$$
\omega\left(\lambda^{2}\right)=\frac{1+h\left(a \lambda^{2}+\alpha+\frac{d}{\lambda^{2}}\right)}{1+h\left(c \lambda^{2}+\beta+\frac{b}{\lambda^{2}}\right)} .
$$

Note that the Lax matrix $V_{n}(3.22)$ approaches a diagonal matrix as $n \rightarrow \pm \infty$ (cf. (3.24)), and $\omega\left(\lambda^{2}\right)$ is equal to the limit value of the ratio between the diagonal elements [3]; this is natural because the $n$-independent overall factor of $V_{n}$ plays no role in the zero-curvature condition (2.6).

For notational convenience, we express the forward time evolution defined by (3.23) with (3.24) as the mapping

$$
f\left(a, b, c, d, \alpha+\frac{1}{h}, \beta+\frac{1}{h}\right):\left(q_{n}, r_{n}\right) \mapsto\left(\widetilde{q}_{n}, \widetilde{r}_{n}\right) .
$$

Note that only the ratio of the six arguments in $f$ matters in identifying the corresponding flow as well as its linear part. Let us discuss how to factorize this mapping into a composition of simpler mappings belonging to the same class. A rigorous and persuasive discussion can be given on the basis of the scattering data used in the inverse scattering formalism. The scattering data are space-independent quantities that have very simple time dependences determined by the dispersion relation, typically, $\widetilde{R}(\lambda)=\omega\left(\lambda^{2}\right) R(\lambda)$ [3. Thus, their time evolution is essentially linear and allows a commutative superposition of different flows. Conversely, the decomposition of a complex flow into elementary flows can be uncovered by examining the factorization of the dispersion relation, that is, whether $\omega\left(\lambda^{2}\right)$ can be factorized into a product of simpler quantities such as $\omega_{1}\left(\lambda^{2}\right) \omega_{2}\left(\lambda^{2}\right)$ or $\omega_{1}\left(\lambda^{2}\right) \omega_{2}\left(\lambda^{2}\right) \omega_{3}\left(\lambda^{2}\right)$. If the reader is unfamiliar with the inverse scattering method, the same guideline can be derived by noting the one-to-one correspondence between (the linear part of) a flow and its dispersion relation. One can also understand this guideline "honestly" at the level of the associated linear problem (cf. the second equation in (2.5) and the sentence beneath (3.54)); that is, by considering whether the Lax matrix $V_{n}$ can be factorized into a product of simpler matrices such as $V_{n}^{(2)}(\lambda) V_{n}^{(1)}(\lambda)$ or $V_{n}^{(3)}(\lambda) V_{n}^{(2)}(\lambda) V_{n}^{(1)}(\lambda)[1,6,47,19]$. 
By elementary computations, the right-hand side of (3.54) can be factorized into the product of two or three simpler quantities (as functions of $\lambda$ ) as follows:

$$
\begin{aligned}
& \frac{1+h\left(a \lambda^{2}+\alpha+\frac{d}{\lambda^{2}}\right)}{1+h\left(c \lambda^{2}+\beta+\frac{b}{\lambda^{2}}\right)} \\
= & \frac{1+h \alpha+\sqrt{(1+h \alpha)^{2}-4 h^{2} a d}+2 h a \lambda^{2}}{1+h \beta+\sqrt{(1+h \beta)^{2}-4 h^{2} b c}+\frac{2 h b}{\lambda^{2}}} \times \frac{1+\frac{2 h d / \lambda^{2}}{1+h \alpha+\sqrt{(1+h \alpha)^{2}-4 h^{2} a d}}}{1+\frac{2 h c \lambda^{2}}{1+h \beta+\sqrt{(1+h \beta)^{2}-4 h^{2} b c}}} \\
= & \frac{1+\frac{2 h a \lambda^{2}}{1+h \alpha+\sqrt{(1+h \alpha)^{2}-4 h^{2} a d}}}{1+\frac{2 h b / \lambda^{2}}{1+h \beta+\sqrt{(1+h \beta)^{2}-4 h^{2} b c}}} \times \frac{1+h \alpha+\sqrt{(1+h \alpha)^{2}-4 h^{2} a d}+\frac{2 h d}{\lambda^{2}}}{1+h \beta+\sqrt{(1+h \beta)^{2}-4 h^{2} b c}+2 h c \lambda^{2}} \\
= & \frac{1+h \alpha+\sqrt{(1+h \alpha)^{2}-4 h^{2} a d}}{1+h \beta+\sqrt{(1+h \beta)^{2}-4 h^{2} b c}} \times \frac{2 h a \lambda^{2}}{1+\frac{2 h b / \lambda^{2}}{1+h \beta+\sqrt{(1+h \beta)^{2}-4 h^{2} b c}}} \\
& \times \frac{1+\frac{2 h d / \lambda^{2}}{1+h \alpha+\sqrt{(1+h \alpha)^{2}-4 h^{2} a d}}}{1+\frac{2 h c \lambda^{2}}{1+h \beta+\sqrt{(1+h \beta)^{2}-4 h^{2} b c}}}
\end{aligned}
$$

Thus, the aforementioned guideline implies the corresponding decomposition of the mapping $f\left(a, b, c, d, \alpha+\frac{1}{h}, \beta+\frac{1}{h}\right)$ into simpler ones, i.e.,

$$
\begin{aligned}
& f\left(a, b, c, d, \alpha+\frac{1}{h}, \beta+\frac{1}{h}\right) \\
= & f\left(a, b, 0,0, \alpha^{\prime}+\frac{1}{h}, \beta^{\prime}+\frac{1}{h}\right) \circ f\left(0,0, c^{\prime}, d^{\prime}, \frac{1}{h}, \frac{1}{h}\right) \\
= & f\left(a^{\prime}, b^{\prime}, 0,0, \frac{1}{h}, \frac{1}{h}\right) \circ f\left(0,0, c, d, \alpha^{\prime}+\frac{1}{h}, \beta^{\prime}+\frac{1}{h}\right) \\
= & f\left(0,0,0,0, \alpha^{\prime}+\frac{1}{h}, \beta^{\prime}+\frac{1}{h}\right) \circ f\left(a^{\prime}, b^{\prime}, 0,0, \frac{1}{h}, \frac{1}{h}\right) \circ f\left(0,0, c^{\prime}, d^{\prime}, \frac{1}{h}, \frac{1}{h}\right),
\end{aligned}
$$

where

$$
\begin{array}{ll}
a^{\prime}:=\frac{2 a}{1+h \alpha+\sqrt{(1+h \alpha)^{2}-4 h^{2} a d}}, \quad b^{\prime}:=\frac{2 b}{1+h \beta+\sqrt{(1+h \beta)^{2}-4 h^{2} b c}}, \\
c^{\prime}:=\frac{2 c}{1+h \beta+\sqrt{(1+h \beta)^{2}-4 h^{2} b c}}, \quad d^{\prime}:=\frac{2 d}{1+h \alpha+\sqrt{(1+h \alpha)^{2}-4 h^{2} a d}}, \\
\alpha^{\prime}:=\frac{\sqrt{(1+h \alpha)^{2}-4 h^{2} a d}-1+h \alpha}{2 h}, & \beta^{\prime}:=\frac{\sqrt{(1+h \beta)^{2}-4 h^{2} b c}-1+h \beta}{2 h} .
\end{array}
$$


Note that the order of composition in (3.56) does not matter, as the order of multiplication in (3.55) does not matter. Interestingly, the time evolution defined on the six points $(n+i, m+j), i=-1,0,1$ and $j=0,1$, is now written as the composition of two time evolutions, each of which is a previously described four-point scheme, and, if any (cf. (3.56c) ), one trivial time evolution. Moreover, if $h$ is real, $b=a^{*}, d=c^{*}$, and $\beta=\alpha^{*}$, the original mapping $f\left(a, b, c, d, \alpha+\frac{1}{h}, \beta+\frac{1}{h}\right)$ allows the reduction of the complex conjugate $r_{n}=\sigma q_{n}^{*}(\sigma$ : real $)$, and this reduction is consistent with every component mapping involved in (3.56).

Using the dispersion relation (3.54), we can readily find the inverse mapping of $f\left(a, b, c, d, \alpha+\frac{1}{h}, \beta+\frac{1}{h}\right)$. Indeed, the trivial identity

$$
\frac{1+h\left(a \lambda^{2}+\alpha+\frac{d}{\lambda^{2}}\right)}{1+h\left(c \lambda^{2}+\beta+\frac{b}{\lambda^{2}}\right)} \times \frac{1+h\left(c \lambda^{2}+\beta+\frac{b}{\lambda^{2}}\right)}{1+h\left(a \lambda^{2}+\alpha+\frac{d}{\lambda^{2}}\right)}=1
$$

implies the nontrivial formula

$$
f^{-1}\left(a, b, c, d, \alpha+\frac{1}{h}, \beta+\frac{1}{h}\right)=f\left(c, d, a, b, \beta+\frac{1}{h}, \alpha+\frac{1}{h}\right) .
$$

In particular, this confirms the already mentioned correspondence between the case $a=b=0$ and the case $c=d=0$ through the time reflection.

Actually, the right-hand side of (3.54) is already the product of the numerator and the inverse of the denominator; this implies the decomposition

$$
\begin{aligned}
f\left(a, b, c, d, \alpha+\frac{1}{h}, \beta+\frac{1}{h}\right) & =f\left(a, 0,0, d, \alpha+\frac{1}{h}, \frac{1}{h}\right) \circ f\left(0, b, c, 0, \frac{1}{h}, \beta+\frac{1}{h}\right) \\
& =f\left(0, b, c, 0, \frac{1}{h}, \beta+\frac{1}{h}\right) \circ f\left(a, 0,0, d, \alpha+\frac{1}{h}, \frac{1}{h}\right) .
\end{aligned}
$$

Thus, using this formula, one can further decompose (3.56) into "elementary" flows (cf. [1, 7, 19]); however, such flows do not maintain the complex conjugacy relation between $q_{n}$ and $r_{n}$, which may become a serious bottleneck in practical applications.

\subsection{The Volterra lattice}

The Volterra lattice

$$
u_{n, t}=u_{n}\left(u_{n+1}-u_{n-1}\right)
$$

is obtained from the Ablowitz-Ladik lattice (3.19) through the reduction $a=b=1, q_{n}=u_{n}-1$, and $r_{n}=-1$. Thus, the time discretization of the Volterra lattice can be obtained from the discrete-time Ablowitz-Ladik lattice in subsection 3.2 through the reduction $q_{n}=u_{n}-1$ and $r_{n}=-1$ together with $a=b, \alpha_{n}=\beta_{n+1}$, and $c_{n}=d_{n}$. The discrepancy between this 
reduction and the decaying boundary conditions (3.24) is nonessential in this regard. However, in the following, we prefer to consider the Volterra lattice independently as an interesting example. The Lax pair for the continuoustime Volterra lattice (3.57) is given by [1, 42]

$$
\begin{aligned}
L_{n} & =\left[\begin{array}{cc}
\lambda & u_{n} \\
-1 & 0
\end{array}\right], \\
M_{n} & =\left[\begin{array}{cc}
\lambda^{2}+u_{n} & \lambda u_{n} \\
-\lambda & u_{n-1}
\end{array}\right] .
\end{aligned}
$$

Indeed, the substitution of (3.58) into the zero-curvature condition (2.2) results in (3.57). form:

In the discrete-time case, we assume the Lax matrix $V_{n}$ of the following

$$
V_{n}=I+h\left[\begin{array}{cc}
\lambda^{2} a+\alpha_{n}+\widetilde{u}_{n} b_{n} & \lambda\left(a u_{n}+\widetilde{u}_{n} b_{n}\right) \\
-\lambda\left(a+b_{n}\right) & -\lambda^{2} b_{n}+\alpha_{n-1}+\widetilde{u}_{n-1} b_{n-1}
\end{array}\right] .
$$

Here, $\alpha_{n}$ and $b_{n}$ are auxiliary variables. The zero-curvature condition (2.6) for the Lax pair (3.58a) and (3.59) is equivalent to the following system of partial difference equations (cf. [5]):

$$
\left\{\begin{array}{l}
\frac{1}{h}\left(\widetilde{u}_{n}-u_{n}\right)=\alpha_{n+1} u_{n}-\widetilde{u}_{n} \alpha_{n-1}+\widetilde{u}_{n+1} b_{n+1} u_{n}-\widetilde{u}_{n} \widetilde{u}_{n-1} b_{n-1}, \\
\alpha_{n+1}-\alpha_{n}=a\left(u_{n+1}-\widetilde{u}_{n}\right) \\
\widetilde{u}_{n} b_{n}=b_{n+1} u_{n}
\end{array}\right.
$$

The general form (3.60) of the time-discretized Volterra lattice is integrable for matrix-valued dependent variables, but in the following, we consider only the case of scalar dependent variables. In view of (2.9) , we impose the following boundary conditions for $u_{n}, \alpha_{n}$, and $b_{n}$ :

$$
\lim _{n \rightarrow \pm \infty} u_{n}=1, \quad \lim _{n \rightarrow \pm \infty} \alpha_{n}=0, \quad \lim _{n \rightarrow \pm \infty} b_{n}=b .
$$

The boundary value of $u_{n}$ is normalized by scaling $a$ and $b$, and the boundary value of $\alpha_{n}$ is set as zero by redefining $h$. To be precise, the boundary conditions (3.61) contain redundant information. Indeed, it can be shown that the auxiliary variables have the same limit values for $n \rightarrow-\infty$ and $n \rightarrow+\infty$. Therefore, it is sufficient to assume either $\lim _{n \rightarrow-\infty}\left(\alpha_{n}, b_{n}\right)=(0, b)$ or $\lim _{n \rightarrow+\infty}\left(\alpha_{n}, b_{n}\right)=(0, b)$. In the continuum limit of time $h \rightarrow 0$, we obtain $\alpha_{n} \rightarrow a u_{n}-a$ and $b_{n} \rightarrow b$. Thus, in this limit, the time discretization (3.60) reduces to

$$
u_{n, t}=(a+b) u_{n}\left(u_{n+1}-u_{n-1}\right),
$$


which is, if $a+b \neq 0$, equivalent to the Volterra lattice (3.57), up to a redefinition of $t$. Note that in the case $a+b=0$, (3.60) has the trivial solution $\widetilde{u}_{n}=u_{n}, \alpha_{n}=a u_{n}-a, b_{n}=b$.

The determinant of the $2 \times 2$ Lax matrix $L_{n}$ (3.58a) can be immediately computed as det $L_{n}=u_{n}$. Using the recurrence formulas for $\alpha_{n}$ and $b_{n}$ in (3.60), we can rewrite the Lax matrix $V_{n}$ (3.59) as an ultralocal form with respect to the auxiliary variables $\alpha_{n}$ and $b_{n}$. Thus, its $2 \times 2$ determinant is computed as

$$
\begin{aligned}
& \operatorname{det} V_{n}(\lambda) \\
= & {\left[1+h\left(\lambda^{2} a+\alpha_{n}+\widetilde{u}_{n} b_{n}\right)\right]\left[1+h\left(-\lambda^{2} b_{n}+\alpha_{n}-a u_{n}+a \widetilde{u}_{n-1}+b_{n} u_{n-1}\right)\right] } \\
& +h^{2} \lambda^{2}\left(a u_{n}+\widetilde{u}_{n} b_{n}\right)\left(a+b_{n}\right) \\
= & -\lambda^{4} h^{2} a b_{n}+\lambda^{2} h\left[\left(a-b_{n}\right)\left(1+h \alpha_{n}\right)+h a^{2} \widetilde{u}_{n-1}+h a b_{n}\left(u_{n-1}+\widetilde{u}_{n}+u_{n}\right)\right] \\
& +\left[1+h\left(\alpha_{n}+\widetilde{u}_{n} b_{n}\right)\right]\left[1+h\left(\alpha_{n}-a u_{n}+a \widetilde{u}_{n-1}+b_{n} u_{n-1}\right)\right] .
\end{aligned}
$$

Therefore, the equality (2.10) combined with the boundary conditions (3.61) (or the streamlined version as stated above) implies the set of relations

$$
\left\{\begin{array}{l}
b_{n}=b \Lambda_{n}, \\
\left(a-b_{n}\right)\left(1+h \alpha_{n}\right)+h a^{2} \widetilde{u}_{n-1}+h a b_{n}\left(u_{n-1}+\widetilde{u}_{n}+u_{n}\right) \\
=\left(a-b+h a^{2}+3 h a b\right) \Lambda_{n}, \\
{\left[1+h\left(\alpha_{n}+\widetilde{u}_{n} b_{n}\right)\right]\left[1+h\left(\alpha_{n}-a u_{n}+a \widetilde{u}_{n-1}+b_{n} u_{n-1}\right)\right]=(1+h b)^{2} \Lambda_{n} .}
\end{array}\right.
$$

Here, $\Lambda_{n}$ is the quantity that satisfies the recurrence formula

$$
\widetilde{u}_{n} \Lambda_{n}=u_{n} \Lambda_{n+1}
$$

and has the normalized boundary value $\lim _{n \rightarrow \pm \infty} \Lambda_{n}=1$. More precisely, only one of the two conditions $\lim _{n \rightarrow-\infty} \Lambda_{n}=1$ and $\lim _{n \rightarrow+\infty} \Lambda_{n}=1$ is required, in accordance with the boundary conditions for $\alpha_{n}$ and $b_{n}$. Then, the other condition can be confirmed. Thus, $\Lambda_{n}$ can be written explicitly (but globally) as

$$
\Lambda_{n}=\prod_{j=-\infty}^{n-1} \frac{\widetilde{u}_{j}}{u_{j}}=\prod_{j=n}^{+\infty} \frac{u_{j}}{\widetilde{u}_{j}} .
$$

Note that the first equality in (3.62) is valid even if $a=0$ (cf. (3.60) ). The algebraic system (3.62) essentially comprises two nontrivial relations for the two unknowns $\alpha_{n}$ and $\Lambda_{n}$.

Because of the nonzero boundary value of $u_{n}$ (3.61), it is rather cumbersome to identify the linear leading part in (3.60) and the corresponding 
dispersion relation (cf. subsection 3.2). Instead, we can compute the asymptotic form of the Lax matrix $V_{n}$ (3.59) as $n \rightarrow-\infty$ or $n \rightarrow+\infty$ and consider its factorization (cf. [1, 6, 7, 19]). The results imply that the cases $a=0$ and $b=0$ form the basis for the general case; that is, the time evolution for general $a$ and $b$ is equivalent to the order-independent composition of two time evolutions corresponding to $a=0$ and $b=0$, respectively. In addition, these two cases are related to each other through the space/time reflection, as we will see below.

Now, we consider the two fundamental cases: $a=0$ or $b=0$.

- The case $a=0, b \neq 0$.

In this case, the auxiliary variable $\alpha_{n}$ vanishes. Thus, the discrete-time system (3.60) reduces to

$$
\left\{\begin{array}{l}
\frac{1}{h}\left(\widetilde{u}_{n}-u_{n}\right)=b \Lambda_{n+1} u_{n}\left(\widetilde{u}_{n+1}-u_{n-1}\right) \\
\widetilde{u}_{n} \Lambda_{n}=u_{n} \Lambda_{n+1}, \quad \lim _{n \rightarrow-\infty} \Lambda_{n}=1 \text { or } \lim _{n \rightarrow+\infty} \Lambda_{n}=1
\end{array}\right.
$$

Note that in the case $h b=-1$, (3.64) has the trivial solution $\widetilde{u}_{n}=u_{n-1}$, $\Lambda_{n}=1 / u_{n-1}$. The algebraic system (3.62) simplifies to a quadratic equation in $\Lambda_{n}$,

$$
\left(1+h b \widetilde{u}_{n} \Lambda_{n}\right)\left(1+h b u_{n-1} \Lambda_{n}\right)=(1+h b)^{2} \Lambda_{n}
$$

or equivalently,

$$
(h b)^{2} \widetilde{u}_{n} u_{n-1} \Lambda_{n}^{2}-\left[(1+h b)^{2}-h b\left(\widetilde{u}_{n}+u_{n-1}\right)\right] \Lambda_{n}+1=0 .
$$

The asymptotic behavior (2.8) of the Lax matrix $V_{n}$ implies that the proper solution of (3.65) is given by

$$
(1+h b)^{2} \Lambda_{n}=\frac{2}{1-\epsilon\left(\widetilde{u}_{n}+u_{n-1}\right)+\sqrt{\left[1-\epsilon\left(\widetilde{u}_{n}+u_{n-1}\right)\right]^{2}-4 \epsilon^{2} \widetilde{u}_{n} u_{n-1}}},
$$

where $\epsilon:=h b /(1+h b)^{2}$. Thus, if $h b \in \mathbb{R}$, then $\epsilon \leq 1 / 4$. In this case, the local expression (3.66) is valid only if $-1<h b<1$, which covers the range $\epsilon<1 / 4$. The case $h b=1$, corresponding to $\epsilon=1 / 4$, involves a subtle sign problem of the square root, which we do not discuss here. If $(h b)^{2}>1$, (3.66) is inconsistent with the boundary conditions, and the other solution of (3.65) should be adopted. In any case, the boundary conditions for $u_{n}$ imply that $\lim _{n \rightarrow-\infty} \Lambda_{n}=\lim _{n \rightarrow+\infty} \Lambda_{n}=1$. As $h b$ approaches 1 , more restrictive conditions have to be imposed on the $u_{n}$ to ensure a consistent choice of the solution of (3.65). 
Substituting (3.66) into the first equation in (3.64), we obtain a time discretization of the Volterra lattice (3.57) in the local form,

$$
\frac{\widetilde{u}_{n}-u_{n}}{\epsilon}=\frac{2 u_{n}\left(\widetilde{u}_{n+1}-u_{n-1}\right)}{1-\epsilon\left(\widetilde{u}_{n+1}+u_{n}\right)+\sqrt{\left[1-\epsilon\left(\widetilde{u}_{n+1}+u_{n}\right)\right]^{2}-4 \epsilon^{2} \widetilde{u}_{n+1} u_{n}}} .
$$

Thus, the value of $\widetilde{u}_{n}$ is uniquely determined by $u_{n}, \widetilde{u}_{n+1}$, and $u_{n-1}$. Note that (3.67) can be rewritten as

$$
\frac{\widetilde{u}_{n}-u_{n}}{\epsilon}=\frac{2\left(\widetilde{u}_{n+1} \widetilde{u}_{n}-u_{n} u_{n-1}\right)}{1+\epsilon\left(\widetilde{u}_{n+1}-u_{n}\right)+\sqrt{\left[1+\epsilon\left(\widetilde{u}_{n+1}-u_{n}\right)\right]^{2}-4 \epsilon \widetilde{u}_{n+1}}} .
$$

If $h b \in \mathbb{R}$ and the $u_{n}$ are nonzero and real-valued at the initial time, then (3.64) implies that the auxiliary variable $\Lambda_{n}$ is always real-valued. Consequently, the discriminant of the quadratic equation (3.65) must be nonnegative. Thus, if $\epsilon<1 / 4, u_{n}$ approaches 1 sufficiently smoothly and fast as $n \rightarrow \pm \infty$, and the $u_{n}$ are close to 1 at the initial time, then the realvaluedness of $u_{n}$ is preserved under the time evolution of the discrete-time Volterra lattice (3.67) or (3.68).

To express the backward time evolution explicitly, we only have to replace $\Lambda_{n+1} u_{n}$ in the first equation of (3.64) with $\Lambda_{n} \widetilde{u}_{n}$ and then substitute the local expression (3.66). The resulting equation is

$$
\frac{\widetilde{u}_{n}-u_{n}}{\epsilon}=\frac{2 \widetilde{u}_{n}\left(\widetilde{u}_{n+1}-u_{n-1}\right)}{1-\epsilon\left(\widetilde{u}_{n}+u_{n-1}\right)+\sqrt{\left[1-\epsilon\left(\widetilde{u}_{n}+u_{n-1}\right)\right]^{2}-4 \epsilon^{2} \widetilde{u}_{n} u_{n-1}}} .
$$

- The case $b=0, a \neq 0$.

In this case, the auxiliary variable $b_{n}$ vanishes. Thus, the discrete-time system (3.60) reduces to

$$
\left\{\begin{array}{l}
\frac{1}{h}\left(\widetilde{u}_{n}-u_{n}\right)+\alpha_{n}\left(\widetilde{u}_{n}-u_{n}\right)=a\left(u_{n+1} u_{n}-\widetilde{u}_{n} \widetilde{u}_{n-1}\right), \\
\alpha_{n+1}-\alpha_{n}=a\left(u_{n+1}-\widetilde{u}_{n}\right), \quad \lim _{n \rightarrow-\infty} \alpha_{n}=0 \text { or } \lim _{n \rightarrow+\infty} \alpha_{n}=0 .
\end{array}\right.
$$

Note that in the case $h a=-1$, (3.70) has the trivial solution $1+h \alpha_{n}=u_{n+1}$, $\widetilde{u}_{n}=u_{n+2}$. Thus, we can assume $h a \neq-1$. The algebraic system (3.62) simplifies to

$$
\left\{\begin{array}{l}
1+h \alpha_{n}+h a \widetilde{u}_{n-1}=(1+h a) \Lambda_{n} \\
\left(1+h \alpha_{n}\right)\left(1+h \alpha_{n}-h a u_{n}+h a \widetilde{u}_{n-1}\right)=\Lambda_{n}
\end{array}\right.
$$


By eliminating $\Lambda_{n}$, (3.71) reduces to a quadratic equation in $1+h \alpha_{n}$,

$$
(1+h a)\left(1+h \alpha_{n}\right)^{2}-\left[1+h a(1+h a)\left(u_{n}-\widetilde{u}_{n-1}\right)\right]\left(1+h \alpha_{n}\right)-h a \widetilde{u}_{n-1}=0 .
$$

The proper solution of this quadratic equation is given by

$$
1+h \alpha_{n}=\frac{1+F_{n}-G_{n}+\sqrt{\left(1+F_{n}-G_{n}\right)^{2}+4 G_{n}}}{2(1+h a)},
$$

with

$$
\begin{aligned}
F_{n} & :=h a(1+h a) u_{n}, \\
G_{n} & :=h a(1+h a) \widetilde{u}_{n-1} .
\end{aligned}
$$

When $h a \in \mathbb{R}$, the local expression (3.72) is valid only if $h a>-1 / 2$; the borderline case $h a=-1 / 2$ is excluded from our consideration. If $h a<-1 / 2$, the other solution of the quadratic equation should be employed. Unless $h a=-1 / 2$, a unified expression for $1+h \alpha_{n}$,

$$
1+h \alpha_{n}=\frac{1+F_{n}-G_{n}+(1+2 h a) \sqrt{\left(\frac{1+F_{n}-G_{n}}{1+2 h a}\right)^{2}+\frac{4 G_{n}}{(1+2 h a)^{2}}}}{2(1+h a)}
$$

can resolve the sign problem of the square root, but it is unwieldy and looks unattractive. Note that the boundary conditions for $u_{n}$ imply that $\lim _{n \rightarrow-\infty} \alpha_{n}=\lim _{n \rightarrow+\infty} \alpha_{n}=0$, and consequently, $\lim _{n \rightarrow-\infty} \Lambda_{n}=\lim _{n \rightarrow+\infty} \Lambda_{n}=1$.

Substituting the local expression (3.72) for $1+h \alpha_{n}$ into the first equation of (3.70), we obtain a time discretization of the Volterra lattice (3.57). In terms of the new parameter $\delta:=h a(1+h a)$, we can write this time discretization as

$$
\frac{\widetilde{u}_{n}-u_{n}}{\delta}=\frac{2\left(u_{n+1} u_{n}-\widetilde{u}_{n} \widetilde{u}_{n-1}\right)}{1+\delta\left(u_{n}-\widetilde{u}_{n-1}\right)+\sqrt{\left[1+\delta\left(u_{n}-\widetilde{u}_{n-1}\right)\right]^{2}+4 \delta \widetilde{u}_{n-1}}} .
$$

Thus, $\widetilde{u}_{n}$ is uniquely determined by $u_{n}, u_{n+1}$, and $\widetilde{u}_{n-1}$. Note that if $h a>-1 / 2$, then $\delta>-1 / 4$. If this inequality is satisfied, $u_{n}$ approaches 1 sufficiently smoothly and fast as $n \rightarrow \pm \infty$, and the $u_{n}$ are close to 1 at the initial time, then the real-valuedness of $u_{n}$ is preserved under the time evolution (3.73).

To obtain the backward time evolution explicitly, we rewrite $\alpha_{n}$ in the first equation of (3.70) as $\alpha_{n+1}-a\left(u_{n+1}-\widetilde{u}_{n}\right)$ and then substitute the local expression (3.72). The resulting equation is

$$
\frac{\widetilde{u}_{n}-u_{n}}{\delta}=\frac{2\left(u_{n+1} u_{n}-\widetilde{u}_{n} \widetilde{u}_{n-1}\right)}{1-\delta\left(u_{n+1}-\widetilde{u}_{n}\right)+\sqrt{\left[1+\delta\left(u_{n+1}-\widetilde{u}_{n}\right)\right]^{2}+4 \delta \widetilde{u}_{n}}} .
$$


It is easy to see that (3.73) is equivalent to (3.68) (or (3.69)) through the space (or time) reflection and the identification $\delta \leftrightarrow-\epsilon$. In the same manner, (3.74) can be identified with (3.68) through the time reflection. Thus, the forward/backward time evolution in the case $b=0$ corresponds to the backward/forward time evolution in the case $a=0$, up to a redefinition of the parameters.

Ultradiscretization [12,13]. We propose an ultradiscrete analogue of the timediscretized Volterra lattice in the case $b=0$. For the forward time evolution, we first rewrite (3.73) as

$$
\frac{\widetilde{u}_{n}}{u_{n}}=\frac{1+\delta\left(u_{n}-\widetilde{u}_{n-1}\right)+2 \delta u_{n+1}+\sqrt{1+2 \delta\left(u_{n}+\widetilde{u}_{n-1}\right)+\delta^{2}\left(u_{n}-\widetilde{u}_{n-1}\right)^{2}}}{1+\delta\left(u_{n}+\widetilde{u}_{n-1}\right)+\sqrt{1+2 \delta\left(u_{n}+\widetilde{u}_{n-1}\right)+\delta^{2}\left(u_{n}-\widetilde{u}_{n-1}\right)^{2}}} .
$$

Then, we assume $\delta>0$ so that the positivity of the dependent variable, $u_{n}>0, \forall n \in \mathbb{Z}$, can be preserved in the time evolution. By reparametrizing the parameter and the dependent variable as $\delta=: \exp (-L / \varepsilon)$ and $u_{n}=: \exp \left(U_{n} / \varepsilon\right)$, respectively, and taking the logarithm, the above equation becomes

$$
\begin{aligned}
\widetilde{U}_{n}-U_{n}= & \varepsilon \log \left[1+\mathrm{e}^{\frac{U_{n}-L}{\varepsilon}}-\mathrm{e}^{\frac{\widetilde{U}_{n-1}-L}{\varepsilon}}+2 \mathrm{e}^{\frac{U_{n+1}-L}{\varepsilon}}+\sqrt{X_{n}}\right] \\
& -\varepsilon \log \left[1+\mathrm{e}^{\frac{U_{n}-L}{\varepsilon}}+\mathrm{e}^{\frac{\widetilde{U}_{n-1}-L}{\varepsilon}}+\sqrt{X_{n}}\right] \\
X_{n}:= & 1+2 \mathrm{e}^{\frac{U_{n}-L}{\varepsilon}}+2 \mathrm{e}^{\frac{\widetilde{U}_{n-1}-L}{\varepsilon}}+\left(\mathrm{e}^{\frac{U_{n}-L}{\varepsilon}}-\mathrm{e}^{\frac{\widetilde{U}_{n-1}-L}{\varepsilon}}\right)^{2} .
\end{aligned}
$$

For brevity, the $\varepsilon$-dependence of the $U_{n}$ is suppressed. When taking the limit $\varepsilon \rightarrow+0$, the well-known formula [13, 43, 44],

$$
\lim _{\varepsilon \rightarrow+0} \varepsilon \log \left(\sum_{j=1}^{M} \mathrm{e}^{\frac{A_{j}(\varepsilon)}{\varepsilon}}\right)=\max _{1 \leq j \leq M}\left(A_{j}\right)
$$

where $A_{j}(\varepsilon)$ are real functions allowing the one-sided $\operatorname{limit}_{\lim _{\varepsilon \rightarrow+0}} A_{j}(\varepsilon)=: A_{j}$, does not apply directly; however, the core idea behind this formula is still valid. After simple consideration of the cases $U_{n} \gtreqless \widetilde{U}_{n-1}$ when $U_{n}>L$, (3.76) is shown to reduce, in the limit $\varepsilon \rightarrow+0$, to

$$
\widetilde{U}_{n}-U_{n}=\max \left(f\left(U_{n}, \widetilde{U}_{n-1}\right), U_{n+1}-L\right)-\max \left(0, U_{n}-L, \widetilde{U}_{n-1}-L\right) .
$$


Here, $U_{n}:=\lim _{\varepsilon \rightarrow+0} U_{n}(\varepsilon)$ is a real variable, and the boundary conditions $\lim _{n \rightarrow \pm \infty} u_{n}=1$ translate into $\lim _{n \rightarrow \pm \infty} U_{n}=0$. The function $f\left(U_{n}, \widetilde{U}_{n-1}\right)$ is defined by

$$
\begin{aligned}
f\left(U_{n}, \widetilde{U}_{n-1}\right) & :=\lim _{\varepsilon \rightarrow+0} \varepsilon \log \left[1+\mathrm{e}^{\frac{U_{n}-L}{\varepsilon}}-\mathrm{e}^{\frac{\widetilde{U}_{n-1}-L}{\varepsilon}}+\sqrt{X_{n}}\right] \\
& = \begin{cases}U_{n}-L, & U_{n}>\widetilde{U}_{n-1} \text { and } U_{n}>L \\
\widetilde{U}_{n-1}-U_{n-1}+g\left(\widetilde{U}_{n-2}, U_{n-1}\right), & U_{n}=\widetilde{U}_{n-1}>L \\
0, & U_{n}<\widetilde{U}_{n-1} \text { or } U_{n} \leq L\end{cases} \\
& \geq 0,
\end{aligned}
$$

where $g\left(\widetilde{U}_{n-2}, U_{n-1}\right)$ is defined in a similar manner as

$$
\begin{aligned}
g\left(\widetilde{U}_{n-2}, U_{n-1}\right) & :=\lim _{\varepsilon \rightarrow+0} \varepsilon \log \left[1+\mathrm{e}^{\frac{\widetilde{U}_{n-2}-L}{\varepsilon}}-\mathrm{e}^{\frac{U_{n-1}-L}{\varepsilon}}+\sqrt{X_{n-1}}\right] \\
& = \begin{cases}\widetilde{U}_{n-2}-L, & \widetilde{U}_{n-2}>U_{n-1} \text { and } \widetilde{U}_{n-2}>L \\
f\left(U_{n-2}, \widetilde{U}_{n-3}\right), & \widetilde{U}_{n-2}=U_{n-1}>L \\
0, & \widetilde{U}_{n-2}<U_{n-1} \text { or } \widetilde{U}_{n-2} \leq L \\
& \geq 0 .\end{cases}
\end{aligned}
$$

One might naively think that $f\left(U_{n}, \widetilde{U}_{n-1}\right)$ at $U_{n}=\widetilde{U}_{n-1}>L$ is given by $\left(U_{n}-L\right) / 2$; however, this is not true in general, because the equality $U_{n}(+0)=\widetilde{U}_{n-1}(+0)$ does not imply $U_{n}(\varepsilon) \equiv \widetilde{U}_{n-1}(\varepsilon), 0<\varepsilon \ll 1$. As indicated above, the correct value of $f\left(U_{n}, \widetilde{U}_{n-1}\right)$ can be computed recursively using the formulas

$$
f\left(U_{n+1}, \widetilde{U}_{n}\right)=\widetilde{U}_{n}-U_{n}+g\left(\widetilde{U}_{n-1}, U_{n}\right), \quad g\left(\widetilde{U}_{n}, U_{n+1}\right)=f\left(U_{n}, \widetilde{U}_{n-1}\right) .
$$

We now briefly explain how these formulas can be derived in the most general case, i.e., without any assumptions on the arguments such as $U_{n+1}=\widetilde{U}_{n}$. Note that (3.78) can be regarded as a conservation law. Thus, in view of the zero boundary conditions for $U_{n}$, the following global expressions are valid if $L>0$ :

$$
\begin{aligned}
& f\left(U_{n+1}, \widetilde{U}_{n}\right)=\sum_{j=0}^{\infty}\left(\widetilde{U}_{n-2 j}-U_{n-2 j}\right)=\sum_{j=1}^{\infty}\left(U_{n+2 j}-\widetilde{U}_{n+2 j}\right) \\
& g\left(\widetilde{U}_{n}, U_{n+1}\right)=\sum_{j=0}^{\infty}\left(\widetilde{U}_{n-2 j-1}-U_{n-2 j-1}\right)=\sum_{j=1}^{\infty}\left(U_{n+2 j-1}-\widetilde{U}_{n+2 j-1}\right) .
\end{aligned}
$$


To verify (3.78), we compare (3.75) with (3.63), wherein the expression for $\Lambda_{n}$ is given by the first equation in (3.71) with (3.72). This results in the nontrivial relation

$$
\begin{aligned}
& 1+\delta\left(\widetilde{u}_{n}-u_{n+1}\right)+\sqrt{1+2 \delta\left(u_{n+1}+\widetilde{u}_{n}\right)+\delta^{2}\left(u_{n+1}-\widetilde{u}_{n}\right)^{2}} \\
& =1+\delta\left(u_{n}-\widetilde{u}_{n-1}\right)+\sqrt{1+2 \delta\left(u_{n}+\widetilde{u}_{n-1}\right)+\delta^{2}\left(u_{n}-\widetilde{u}_{n-1}\right)^{2}} .
\end{aligned}
$$

It is an easy exercise to rewrite this relation by rationalizing its numerator as

$$
\begin{aligned}
& 1+\delta\left(u_{n+1}-\widetilde{u}_{n}\right)+\sqrt{1+2 \delta\left(u_{n+1}+\widetilde{u}_{n}\right)+\delta^{2}\left(u_{n+1}-\widetilde{u}_{n}\right)^{2}} \\
& =\frac{\widetilde{u}_{n}}{u_{n}}\left[1+\delta\left(\widetilde{u}_{n-1}-u_{n}\right)+\sqrt{1+2 \delta\left(u_{n}+\widetilde{u}_{n-1}\right)+\delta^{2}\left(u_{n}-\widetilde{u}_{n-1}\right)^{2}}\right] .
\end{aligned}
$$

By substituting $\delta=\exp (-L / \varepsilon)$ and $u_{n}=\exp \left(U_{n} / \varepsilon\right)$, and taking the logarithm, the above two equalities reduce, in the limit $\varepsilon \rightarrow+0$, to the second and first equalities in (3.78), respectively.

The parameter $L$ may depend on the discrete time coordinate $m \in \mathbb{Z}$. The commutativity of the ultradiscrete flows defined by (3.77) for distinct values of $L$ remains to be established. In this connection, we conjecture that (3.77) and its time reversal (see below) comprise an ultradiscrete analogue of the $\mathrm{KdV}$ hierarchy, wherein the parameter $L$ labels each flow in the hierarchy. It is also beyond the scope of this paper to investigate the relationship between the ultradiscretized Volterra lattice (3.77) and the "box and ball system" of Takahashi and Satsuma [45].

For the backward time evolution, we rewrite (3.74) as

$$
\frac{u_{n}}{\widetilde{u}_{n}}=\frac{1+\delta\left(\widetilde{u}_{n}-u_{n+1}\right)+2 \delta \widetilde{u}_{n-1}+\sqrt{1+2 \delta\left(\widetilde{u}_{n}+u_{n+1}\right)+\delta^{2}\left(\widetilde{u}_{n}-u_{n+1}\right)^{2}}}{1+\delta\left(\widetilde{u}_{n}+u_{n+1}\right)+\sqrt{1+2 \delta\left(\widetilde{u}_{n}+u_{n+1}\right)+\delta^{2}\left(\widetilde{u}_{n}-u_{n+1}\right)^{2}}} .
$$

Thus, in the same way as that for the forward time evolution, we obtain an ultradiscrete analogue of (3.74),

$$
U_{n}-\widetilde{U}_{n}=\max \left(g\left(\widetilde{U}_{n}, U_{n+1}\right), \widetilde{U}_{n-1}-L\right)-\max \left(0, \widetilde{U}_{n}-L, U_{n+1}-L\right),
$$

which is related to (3.77) through the combined space and time reflection (cf. (3.74) and (3.73) ). 


\subsection{The modified Volterra lattice}

The modified Volterra lattice

$$
q_{n, t}=\left(1+q_{n}^{2}\right)\left(q_{n+1}-q_{n-1}\right),
$$

where $q_{n}$ is a scalar dependent variable, was introduced by Hirota [46] (also see [4, 47, 48]); it is obtained from the Ablowitz-Ladik system (3.19) through the reduction $a=b=1$ and $r_{n}=-q_{n}$. The overall coefficient of the nonlinear terms, including its sign, is nonessential at the level of the equation and the associated Lax pair, because it can be changed by rescaling $q_{n}$. Note that (3.79) is invariant under the transformation $q_{n} \mapsto(-1)^{n} q_{n}, t \mapsto-t$. The Lax pair for the continuous-time modified Volterra lattice (3.79) is given by

$$
\begin{aligned}
L_{n} & =\left[\begin{array}{cc}
\lambda & q_{n} \\
-q_{n} & \frac{1}{\lambda}
\end{array}\right], \\
M_{n} & =\left[\begin{array}{cc}
\lambda^{2}+q_{n} q_{n-1} & \lambda q_{n}+\frac{1}{\lambda} q_{n-1} \\
-\lambda q_{n-1}-\frac{1}{\lambda} q_{n} & q_{n} q_{n-1}+\frac{1}{\lambda^{2}}
\end{array}\right] .
\end{aligned}
$$

Indeed, the substitution of (3.80) into the zero-curvature condition (2.2) results in (3.79).

In the discrete-time case, we consider the reduction $a=b, \alpha_{n}=\beta_{n}, c_{n}=d_{n}$, and $r_{n}=-q_{n}$ of the Lax matrix $V_{n}(3.22)$. Thus, we obtain

$$
V_{n}=I+h\left[\begin{array}{c|c}
\lambda^{2} a-\widetilde{q}_{n} q_{n-1} c_{n} & \lambda\left(a q_{n}-\widetilde{q}_{n} c_{n}\right) \\
+\alpha_{n}+\frac{1}{\lambda^{2}} c_{n} & +\frac{1}{\lambda}\left(a \widetilde{q}_{n-1}-c_{n} q_{n-1}\right) \\
\hline \lambda\left(-a \widetilde{q}_{n-1}+c_{n} q_{n-1}\right) & \lambda^{2} c_{n}-\widetilde{q}_{n} q_{n-1} c_{n} \\
+\frac{1}{\lambda}\left(-a q_{n}+\widetilde{q}_{n} c_{n}\right) & +\alpha_{n}+\frac{a}{\lambda^{2}}
\end{array}\right],
$$

where $\alpha_{n}$ and $c_{n}$ are the auxiliary variables. The zero-curvature condition (2.6) for the Lax pair (3.80a) and (3.81) amounts to the following system of partial difference equations:

$$
\left\{\begin{aligned}
\frac{1}{h}\left(\widetilde{q}_{n}-q_{n}\right) & -a q_{n+1}+a \widetilde{q}_{n-1}-\alpha_{n+1} q_{n}+\widetilde{q}_{n} \alpha_{n} \\
& +\widetilde{q}_{n+1} c_{n+1}\left(1+q_{n}^{2}\right)-\left(1+\widetilde{q}_{n}^{2}\right) c_{n} q_{n-1}=0 \\
\alpha_{n+1}-\alpha_{n}= & a\left(-\widetilde{q}_{n} \widetilde{q}_{n-1}+q_{n+1} q_{n}\right) \\
\left(1+\widetilde{q}_{n}^{2}\right) c_{n}= & \left(1+q_{n}^{2}\right) c_{n+1}
\end{aligned}\right.
$$

The discussion in subsection 3.2 implies that the general case of $a \neq 0$ and $c_{n} \neq 0$ is equivalent to the composition of two simpler cases: the case $a=0$ 
and $\alpha_{n}=0$ and the case $c_{n}=0$. Moreover, the latter case can be related with the former case through the time reflection. Thus, in this subsection, we only consider the elementary case $a=0, \alpha_{n}=0$, and $c_{n}=-\Lambda_{n}$ under the boundary conditions

$$
\lim _{n \rightarrow \pm \infty} q_{n}=0, \quad \lim _{n \rightarrow \pm \infty} \Lambda_{n}=1 .
$$

Note that the boundary value of $c_{n}$ is set as -1 without any loss of generality; it can be changed to any nonzero value by rescaling the "step size" parameter $h$. To be precise, the boundary conditions (3.83) contain redundant information. Indeed, it can be shown that $\Lambda_{n}$ has the same limit value for $n \rightarrow-\infty$ and $n \rightarrow+\infty$. Therefore, it is sufficient to assume either $\lim _{n \rightarrow-\infty} \Lambda_{n}=1$ or $\lim _{n \rightarrow+\infty} \Lambda_{n}=1$. Now, the general system (3.82) reduces to the simpler system

$$
\left\{\begin{array}{l}
\frac{1}{h}\left(\widetilde{q}_{n}-q_{n}\right)=\left(1+q_{n}^{2}\right) \Lambda_{n+1}\left(\widetilde{q}_{n+1}-q_{n-1}\right), \\
\left(1+\widetilde{q}_{n}^{2}\right) \Lambda_{n}=\left(1+q_{n}^{2}\right) \Lambda_{n+1} .
\end{array}\right.
$$

The second relation in (3.84) implies that the global expressions for the auxiliary variable $\Lambda_{n}$ in terms of the $q_{n}$ are

$$
\Lambda_{n}=\prod_{j=-\infty}^{n-1} \frac{1+\widetilde{q}_{j}^{2}}{1+q_{j}^{2}}=\prod_{j=n}^{+\infty} \frac{1+q_{j}^{2}}{1+\widetilde{q}_{j}^{2}} .
$$

The substitution of each expression into the first relation in (3.84 provides a global-in-space time discretization of the modified Volterra lattice (3.79). If $h \in \mathbb{R}$ and the $q_{n}$ are real-valued at the initial time, then the real-valuedness of $q_{n}$ is preserved under the discrete-time evolution.

Let us resolve this nonlocality. The determinant of the $2 \times 2$ Lax matrix $L_{n}$ (3.80a) is given by $\operatorname{det} L_{n}=1+q_{n}^{2}$, while the determinant of the Lax matrix $V_{n}$ (3.81) with $a=0, \alpha_{n}=0$, and $c_{n}=-\Lambda_{n}$ is computed as

$$
\begin{aligned}
\operatorname{det} V_{n}(\lambda)= & -\left(\lambda^{2}+1 / \lambda^{2}\right) h \Lambda_{n} \\
& +h^{2}\left(1+\widetilde{q}_{n}^{2}\right)\left(1+q_{n-1}^{2}\right) \Lambda_{n}^{2}+2 h \widetilde{q}_{n} q_{n-1} \Lambda_{n}+1 .
\end{aligned}
$$

Thus, the equality (2.10) combined with the boundary conditions (3.83) (or the streamlined version as stated above) leads to the quadratic equation in $\Lambda_{n}$,

$$
h^{2}\left(1+\widetilde{q}_{n}^{2}\right)\left(1+q_{n-1}^{2}\right) \Lambda_{n}^{2}-\left(1+h^{2}-2 h \widetilde{q}_{n} q_{n-1}\right) \Lambda_{n}+1=0 .
$$


By recalling the prescribed asymptotic behavior (2.8) of the Lax matrix $V_{n}$ for small $h$, we obtain the proper solution of this quadratic equation in $\Lambda_{n}$,

$$
\begin{aligned}
\Lambda_{n} & =\frac{2}{1+h^{2}-2 h \widetilde{q}_{n} q_{n-1}+\sqrt{\left(1+h^{2}-2 h \widetilde{q}_{n} q_{n-1}\right)^{2}-4 h^{2}\left(1+\widetilde{q}_{n}^{2}\right)\left(1+q_{n-1}^{2}\right)}} \\
& =\frac{2}{1+h^{2}-2 h \widetilde{q}_{n} q_{n-1}+\sqrt{\left(1-h^{2}\right)^{2}-4 h\left(\widetilde{q}_{n}+h q_{n-1}\right)\left(h \widetilde{q}_{n}+q_{n-1}\right)}} .
\end{aligned}
$$

When $h \in \mathbb{R}$, the local expression (3.86) is valid only if $-1 \leq h \leq 1$. If $h^{2}>1$, the other solution of (3.85) should be used. Unless $h= \pm 1$, a unified expression for $\Lambda_{n}$,

$$
\Lambda_{n}=\frac{2}{1+h^{2}-2 h \widetilde{q}_{n} q_{n-1}+\left(1-h^{2}\right) \sqrt{1-\frac{4 h}{\left(1-h^{2}\right)^{2}}\left(\widetilde{q}_{n}+h q_{n-1}\right)\left(h \widetilde{q}_{n}+q_{n-1}\right)}},
$$

can resolve the sign problem of the square root, but we do not use this form. In any case, the decaying boundary conditions for $q_{n}$ imply that $\lim _{n \rightarrow-\infty} \Lambda_{n}=\lim _{n \rightarrow+\infty} \Lambda_{n}=1$. Thus, the boundary conditions for $\Lambda_{n}$ given in (3.83) are compatible.

Substituting (3.86) into the first equation in (3.84), we obtain a time discretization of the modified Volterra lattice (3.79) in the local form,

$$
\begin{aligned}
& \frac{1}{h}\left(\widetilde{q}_{n}-q_{n}\right) \\
& =\frac{2\left(1+q_{n}^{2}\right)\left(\widetilde{q}_{n+1}-q_{n-1}\right)}{1+h^{2}-2 h \widetilde{q}_{n+1} q_{n}+\sqrt{\left(1-h^{2}\right)^{2}-4 h\left(\widetilde{q}_{n+1}+h q_{n}\right)\left(h \widetilde{q}_{n+1}+q_{n}\right)}} .
\end{aligned}
$$

Note that the right-hand side of (3.87) does not involve $\widetilde{q}_{n}$. Similarly to the continuous-time case, (3.87) (or (3.84)) is invariant under the transformation $q_{n} \mapsto(-1)^{n} q_{n}, h \mapsto-h$. If $-1 \leq h \leq 1$, the real-valuedness of $q_{n}$ is preserved under the discrete-time evolution (3.87); the discriminant in the square root is nonnegative as long as we start with sufficiently small real-valued $q_{n}$ at the initial time. To express the backward time evolution explicitly, we only have to replace $\left(1+q_{n}^{2}\right) \Lambda_{n+1}$ in the first equation of (3.84) with $\left(1+\widetilde{q}_{n}^{2}\right) \Lambda_{n}$ (cf. the second equation) and then substitute the local expression (3.86).

In terms of the new parameter $\delta$ given by $\delta:=h /\left(1+h^{2}\right)$, (3.87) can be rewritten in a slightly more compact form,

$$
\frac{1}{\delta}\left(\widetilde{q}_{n}-q_{n}\right)=\frac{2\left(1+q_{n}^{2}\right)\left(\widetilde{q}_{n+1}-q_{n-1}\right)}{1-2 \delta \widetilde{q}_{n+1} q_{n}+\sqrt{1-4 \delta \widetilde{q}_{n+1} q_{n}-4 \delta^{2}\left(1+\widetilde{q}_{n+1}^{2}+q_{n}^{2}\right)}} .
$$


Note that if $h \in \mathbb{R}($ or $-1 \leq h \leq 1)$, then $-1 / 2 \leq \delta \leq 1 / 2$.

At the "threshold" values of $h, h= \pm 1$, corresponding to $\delta= \pm 1 / 2$, we can extract the square root in (3.87) to obtain a rational mapping. This is connected with the fact that in the cases $h= \pm 1$, the discrete-time system (3.84) under the boundary conditions (3.83) has the trivial solution $\widetilde{q}_{n}=\mp q_{n-1}, \Lambda_{n}=1 /\left(1+q_{n-1}^{2}\right)$ (cf. $\S 4.6$ in [1]). Thus, the discriminant of the quadratic equation (3.85) vanishes at $h= \pm 1$, and the two solutions intersect. To obtain a nontrivial mapping from this observation, we set $h=+1$ in (3.84) and replace $q_{n}$ with $\mathrm{i} w_{n}$, namely,

$$
\left\{\begin{array}{l}
\widetilde{w}_{n}-w_{n}=\left(1-w_{n}^{2}\right) \Lambda_{n+1}\left(\widetilde{w}_{n+1}-w_{n-1}\right), \\
\left(1-\widetilde{w}_{n}^{2}\right) \Lambda_{n}=\left(1-w_{n}^{2}\right) \Lambda_{n+1} .
\end{array}\right.
$$

Moreover, we generalize the boundary conditions (3.83) as

$$
\lim _{n \rightarrow \pm \infty} w_{n}=\gamma, \quad \lim _{n \rightarrow \pm \infty} \Lambda_{n}=\frac{1}{(1+\gamma)^{2}}, \quad \gamma \neq-1
$$

and assume that $\left|w_{n}-\gamma\right|$ is sufficiently small. Thus, the additional condition $\gamma \neq 0$ excludes the trivial time evolution $\widetilde{w}_{n}=-w_{n-1}$. Following the same procedure as above, we obtain the quadratic equation in $\Lambda_{n}$ that can be factorized as

$$
\left[\left(1+\widetilde{w}_{n}\right)\left(1+w_{n-1}\right) \Lambda_{n}-1\right]\left[\left(1-\widetilde{w}_{n}\right)\left(1-w_{n-1}\right) \Lambda_{n}-1\right]=0 .
$$

Substituting its proper solution $\Lambda_{n}=1 /\left[\left(1+\widetilde{w}_{n}\right)\left(1+w_{n-1}\right)\right]$ into (3.89), we obtain a single equation $\left(\widetilde{w}_{n}-1\right)\left(\widetilde{w}_{n+1}+1\right)=\left(w_{n}-1\right)\left(w_{n-1}+1\right)$. By a trivial change of the dependent variable $w_{n}=: 1+2 \nu y_{n}(\nu \neq 0)$, we obtain the well-known "discrete Volterra equation" [17,49],

$$
\widetilde{y}_{n}\left(1+\nu \widetilde{y}_{n+1}\right)=y_{n}\left(1+\nu y_{n-1}\right) \text {. }
$$

Therefore, the discrete-time equation (3.90) belongs to the modified Volterra hierarchy and not the original Volterra hierarchy [1]; this corresponds to the special case where the quadratic equation for the auxiliary variable $\Lambda_{n}$ is factorized to provide a rational solution.

Ultradiscretization [12,13]. We present an intuitively plausible derivation of an ultradiscrete analogue of the time-discretized modified Volterra lattice, although this may not be a unique ultradiscretization. For the forward time 
evolution, we rewrite (3.88) as

$$
\begin{aligned}
\widetilde{q}_{n} & =\frac{q_{n}\left\{1+\epsilon q_{n} q_{n-1}+\sqrt{Y_{n}}\right\}-\epsilon\left(\widetilde{q}_{n+1}-q_{n-1}\right)}{1+\epsilon \widetilde{q}_{n+1} q_{n}+\sqrt{Y_{n}}} \\
& =\frac{q_{n}\left\{1+\epsilon\left(q_{n}+1 / q_{n}\right) q_{n-1}+\sqrt{Y_{n}}-\epsilon \widetilde{q}_{n+1} / q_{n}\right\}}{1+\epsilon \widetilde{q}_{n+1} q_{n}+\sqrt{Y_{n}}},
\end{aligned}
$$

where $\epsilon:=-2 \delta$ and $Y_{n}:=1+2 \epsilon \widetilde{q}_{n+1} q_{n}-\epsilon^{2}\left(1+\widetilde{q}_{n+1}^{2}+q_{n}^{2}\right)$. Note that the equation $\widetilde{q}_{n}=q_{n}$ in the trivial case $\epsilon=0$ preserves the sign of the dependent variable on each lattice site $n \in \mathbb{Z}$. Thus, we consider a class of solutions in the limit $\epsilon \rightarrow+0$ such that the positivity of the dependent variable, $q_{n}>0, \forall n \in \mathbb{Z}$, is preserved in the nontrivial time evolution. We introduce the parametrization $\epsilon=: \exp (-L / \varepsilon), L>0$ and $q_{n, m}=: \rho \exp \left(Q_{n, m} / \varepsilon\right)$, $\rho>0$, where $m \in \mathbb{Z}$ is the discrete time coordinate, which is usually suppressed. The scaling parameter $\rho$ can depend weakly on $n, m$, and $\varepsilon$, but for brevity, we treat it as a constant. Once this parametrization is substituted into (3.91), the solution $\left\{Q_{n, m}\right\}$ of the initial-value problem depends on $\varepsilon$ via the time evolution. We hypothesize that by choosing $\rho$ appropriately, the $\varepsilon$-dependence of $Q_{n}$ in the considered class of solutions becomes negligible as $\varepsilon \rightarrow+0$, and $Q_{n}$ is nonnegative, $Q_{n} \geq 0$. Probably, the simplest way to justify the latter condition is to modify the zero boundary conditions (cf. (3.83) ) to nonzero boundary conditions, $\lim _{n \rightarrow \pm \infty} q_{n}=\rho$, and to assume $q_{n} \geq \rho, \forall n \in \mathbb{Z}$. We are not interested in tracing infinitely long tails of solitons that decay exponentially as $n \rightarrow \pm \infty$; rather, we prefer to extract solitons with compact support in the limit $\varepsilon \rightarrow+0$. This is why we impose the zero boundary conditions $\lim _{n \rightarrow \pm \infty} Q_{n}=0$ on the new variable $Q_{n}$.

The condition $Y_{n} \geq 0$, guaranteed by the nonnegativity of the discriminant of (3.85), requires that

$$
\epsilon q_{n} \leq \widetilde{q}_{n+1}+\sqrt{\left(1-\epsilon^{2}\right)\left(1+\widetilde{q}_{n+1}^{2}\right)} \text { and } \epsilon \widetilde{q}_{n+1} \leq q_{n}+\sqrt{\left(1-\epsilon^{2}\right)\left(1+q_{n}^{2}\right)} \text {. }
$$

Using the parametrization $\epsilon=\exp (-L / \varepsilon)$ and $q_{n}=\rho \exp \left(Q_{n} / \varepsilon\right)$ with $Q_{n} \geq 0$, we can interpret these conditions in the limit $\varepsilon \rightarrow+0$ as $-L \leq \widetilde{Q}_{n+1}-Q_{n} \leq L$. 
By taking the logarithm, (3.91) becomes

$$
\begin{aligned}
\widetilde{Q}_{n}= & Q_{n}+\varepsilon \log \left[1+\mathrm{e}^{\frac{Q_{n}+Q_{n-1}-L}{\varepsilon}}\left(\rho^{2}+\mathrm{e}^{-\frac{2 Q_{n}}{\varepsilon}}\right)+\sqrt{Y_{n}}-\mathrm{e}^{\frac{\tilde{Q}_{n+1}-Q_{n}-L}{\varepsilon}}\right] \\
& -\varepsilon \log \left[1+\rho^{2} \mathrm{e}^{\frac{\widetilde{Q}_{n+1}+Q_{n}-L}{\varepsilon}}+\sqrt{Y_{n}}\right] \\
Y_{n}= & 1-\mathrm{e}^{-\frac{2 L}{\varepsilon}}+\rho^{2} \mathrm{e}^{\frac{\widetilde{Q}_{n+1}+Q_{n}-L}{\varepsilon}}\left(2-\mathrm{e}^{\frac{\widetilde{Q}_{n+1}-Q_{n}-L}{\varepsilon}}-\mathrm{e}^{\frac{Q_{n}-\widetilde{Q}_{n+1}-L}{\varepsilon}}\right)
\end{aligned}
$$

Taking the aforementioned assumptions into account, (3.92) reduces in the limit $\varepsilon \rightarrow+0$ to the following equation:

$$
\begin{aligned}
\widetilde{Q}_{n}= & Q_{n}+\max \left(0, Q_{n}+Q_{n-1}-L, \frac{\widetilde{Q}_{n+1}+Q_{n}-L}{2}\right) \\
& -\max \left(0, \widetilde{Q}_{n+1}+Q_{n}-L\right) .
\end{aligned}
$$

As mentioned above, the boundary conditions $\lim _{n \rightarrow \pm \infty} Q_{n}=0$ are assumed. It is desirable to confirm for each solution that the conditions $Q_{n} \geq 0$ and $\left|\widetilde{Q}_{n+1}-Q_{n}\right| \leq L$ hold true in the time evolution. Note that the parameter $L$ may depend on the discrete time. It is hoped that the issue of the commutativity of the ultradiscrete flows defined by (3.93) for distinct values of $L$ will be investigated. In this regard, we expect that (3.93) and its time reversal (see below) will comprise an ultradiscrete analogue of the mKdV hierarchy, wherein the parameter $L$ labels each flow in the hierarchy. The ultradiscrete equation (3.93) is "linear" in the sense that it is invariant under the rescaling $Q_{n} \mapsto k Q_{n}, L \mapsto k L, k>0$. Thus, if $L$ is a time-independent constant, it is possible to fix $L$ at unity. However, this normalization often changes an integer-valued $Q_{n}$ to a fractional value; thus, we rather prefer to leave $L$ as a free parameter. Our derivation of the ultradiscrete modified Volterra lattice (3.93) is more or less intuitive and is not mathematically rigorous. A more detailed treatment of all the terms in the numerator of (3.91) may lead to a more complicated ultradiscrete equation, but we prefer the relatively simple equation (3.93). Fortunately, for the specific examples that we considered, (3.93) allows the "stable" propagation of solitons and their elastic (but nontrivial) collisions; thus, (at least some of) the integrability properties appear to be retained in this ultradiscretization.

The backward time evolution of the time-discretized modified Volterra lattice is obtained from (3.88) through the combined space and time reflection $n \rightarrow-n, m \rightarrow-m$. Thus, its ultradiscrete analogue is obtained from (3.93) in the same manner. 


\subsection{The lattice Heisenberg ferromagnet model}

The lattice Heisenberg ferromagnet model was proposed in 1982 by several different authors [50 53]. The equation of motion for this semi-discrete model can be derived almost systematically using either Ishimori's approach [51] based on a gauge transformation from the Ablowitz-Ladik lattice or the $r$ matrix formalism based on the fundamental Poisson bracket relation [50, 53]. However, for a concise and easy-to-understand derivation, we use a more heuristic approach based on the zero-curvature representation.

We start with the Lax pair of the following form in the semi-discrete case:

$$
\begin{aligned}
L_{n} & =I+\lambda S_{n}, \\
M_{n} & =\frac{\lambda}{1-\lambda^{2}} A_{n} L_{n},
\end{aligned}
$$

where the conditions $\left(S_{n}\right)^{2}=I$ and $A_{n} S_{n}=S_{n-1} A_{n}$ are assumed. The latter condition guarantees the useful relation $A_{n} L_{n}=L_{n-1} A_{n}$. Thus, substituting the Lax pair (3.94) into the zero-curvature condition (2.2), we obtain

$$
\left(L_{n}^{-1}\right)_{t}+\frac{\lambda}{1-\lambda^{2}}\left(A_{n+1}-A_{n}\right)=O .
$$

Noting the identity $\left(I+\lambda S_{n}\right)\left(I-\lambda S_{n}\right)=\left(1-\lambda^{2}\right) I$, this results in

$$
S_{n, t}=A_{n+1}-A_{n} .
$$

Because $\left(S_{n}\right)^{2}=I$ and $A_{n} S_{n}=S_{n-1} A_{n}$, (3.95) implies the relation

$$
A_{n+1}\left(S_{n+1}+S_{n}\right)=\left(S_{n}+S_{n-1}\right) A_{n} .
$$

Thus, we choose $A_{n}$ as

$$
A_{n}=2 \mathrm{i} a S_{n-1}\left(S_{n}+S_{n-1}\right)^{-1}+2 b\left(S_{n}+S_{n-1}\right)^{-1},
$$

so that the above relation is automatically satisfied. Here, $a$ and $b$ are $n$ independent scalars, but they may depend on the time variable $t$. Substituting this expression for $A_{n}$ into (3.95), we obtain

$$
S_{n, t}=\boldsymbol{\Delta}_{n}^{+}\left[2 \mathrm{i} a S_{n-1}\left(S_{n}+S_{n-1}\right)^{-1}+2 b\left(S_{n}+S_{n-1}\right)^{-1}\right],
$$

where $\boldsymbol{\Delta}_{n}^{+}$is the forward difference operator in the discrete space (cf. (2.4)). Note that the evolution equation (3.96) is consistent with the condition 
$\left(S_{n}\right)^{2}=I$ for a general $l \times l$ matrix $S_{n}$. We now consider the simplest nontrivial case of $l=2$ and express $S_{n}$ in terms of the Pauli matrices as

$$
\begin{aligned}
S_{n} & =\sum_{j=1}^{3} S_{n}^{(j)} \sigma_{j}\left(=: \boldsymbol{\sigma} \cdot \boldsymbol{S}_{n}\right) \\
& =\left[\begin{array}{cc}
S_{n}^{(3)} & S_{n}^{(1)}-\mathrm{i} S_{n}^{(2)} \\
S_{n}^{(1)}+\mathrm{i} S_{n}^{(2)} & -S_{n}^{(3)}
\end{array}\right] .
\end{aligned}
$$

Here, $\boldsymbol{S}_{n}=\left(S_{n}^{(1)}, S_{n}^{(2)}, S_{n}^{(3)}\right)$ is a unit vector, i.e., $\left\langle\boldsymbol{S}_{n}, \boldsymbol{S}_{n}\right\rangle=1$. Because $2\left(S_{n}+S_{n-1}\right)^{-1}=\left(S_{n}+S_{n-1}\right) /\left(1+\left\langle\boldsymbol{S}_{n}, \boldsymbol{S}_{n-1}\right\rangle\right)$ [51], (3.96) reduces to a single vector equation involving both the scalar product and the vector product,

$$
\boldsymbol{S}_{n, t}=\boldsymbol{\Delta}_{n}^{+}\left[a \frac{\boldsymbol{S}_{n} \times \boldsymbol{S}_{n-1}}{1+\left\langle\boldsymbol{S}_{n}, \boldsymbol{S}_{n-1}\right\rangle}+b \frac{\boldsymbol{S}_{n}+\boldsymbol{S}_{n-1}}{1+\left\langle\boldsymbol{S}_{n}, \boldsymbol{S}_{n-1}\right\rangle}\right], \quad\left\langle\boldsymbol{S}_{n}, \boldsymbol{S}_{n}\right\rangle=1
$$

The case $b=0$ gives the lattice Heisenberg ferromagnet model [51,52], while the case $a=0$ corresponds to its simplest higher symmetry [54].

Let us examine the discrete-time case. We mainly consider the time discretization of the reduced system (3.98), and not the general matrix system (3.96), because the latter problem is expected to be too complicated. We start with the Lax matrix $V_{n}$ of the following form:

$$
V_{n}=I+h \frac{\lambda}{1-\lambda^{2}} A_{n} L_{n}
$$

where the conditions $\left(S_{n}\right)^{2}=I$ and $A_{n} S_{n}=\widetilde{S}_{n-1} A_{n}$ are assumed. The latter condition guarantees the useful relation $A_{n} L_{n}=\widetilde{L}_{n-1} A_{n}$. Thus, substituting the Lax pair, (3.94a) and (3.99), into the zero-curvature condition (2.6), we obtain

$$
\frac{1}{h}\left(\widetilde{L}_{n}^{-1}-L_{n}^{-1}\right)+\frac{\lambda}{1-\lambda^{2}}\left(A_{n+1}-A_{n}\right)=O
$$

or equivalently,

$$
\frac{1}{h}\left(\widetilde{S}_{n}-S_{n}\right)=A_{n+1}-A_{n} .
$$

Under the condition $\left(S_{n}\right)^{2}=I$, the relation $A_{n} S_{n}=\widetilde{S}_{n-1} A_{n}$ is automatically satisfied if $A_{n}$ takes the general form $A_{n}=B_{n} S_{n}+\widetilde{S}_{n-1} B_{n}$. However, in analogy with the semi-discrete case, we employ a more specific form of $A_{n}$,

$$
A_{n}=2 \mathrm{i} a_{n} \widetilde{S}_{n-1}\left(S_{n}+\widetilde{S}_{n-1}\right)^{-1}+2 b_{n}\left(S_{n}+\widetilde{S}_{n-1}\right)^{-1}
$$


which also satisfies the relation $A_{n} S_{n}=\widetilde{S}_{n-1} A_{n}$. Here, the scalar unknowns $a_{n}$ and $b_{n}$ are auxiliary variables. Substituting this expression for $A_{n}$ into (3.100), we obtain

$$
\frac{1}{h}\left(\widetilde{S}_{n}-S_{n}\right)=\Delta_{n}^{+}\left[2 \mathrm{i} a_{n} \widetilde{S}_{n-1}\left(S_{n}+\widetilde{S}_{n-1}\right)^{-1}+2 b_{n}\left(S_{n}+\widetilde{S}_{n-1}\right)^{-1}\right]
$$

It only remains necessary to fix the auxiliary variables $a_{n}$ and $b_{n}$. To this end, we recall that the time evolution determined by (3.101) has to be consistent with the condition $\left(S_{n}\right)^{2}=I$. In the following, we focus on the case of the $2 \times 2$ matrix $S_{n}$ given by (3.97). The requirement $\operatorname{tr} S_{n}=0$ results in the $n$-independence of $a_{n}$, thus we set $a_{n}=a$. Therefore, (3.101) reduces to

$$
\frac{1}{h}\left(\widetilde{\boldsymbol{S}}_{n}-\boldsymbol{S}_{n}\right)=\boldsymbol{\Delta}_{n}^{+}\left[a \frac{\boldsymbol{S}_{n} \times \widetilde{\boldsymbol{S}}_{n-1}}{1+\left\langle\boldsymbol{S}_{n}, \widetilde{\boldsymbol{S}}_{n-1}\right\rangle}+b_{n} \frac{\boldsymbol{S}_{n}+\widetilde{\boldsymbol{S}}_{n-1}}{1+\left\langle\boldsymbol{S}_{n}, \widetilde{\boldsymbol{S}}_{n-1}\right\rangle}\right] .
$$

To fix $b_{n}$, we invoke the procedure presented in section 2. we assume the boundary conditions

$$
\lim _{n \rightarrow-\infty}\left\langle\boldsymbol{S}_{n}, \widetilde{\boldsymbol{S}}_{n-1}\right\rangle=1, \quad \lim _{n \rightarrow-\infty} b_{n}=b .
$$

Because $\left\langle\boldsymbol{S}_{n}, \boldsymbol{S}_{n}\right\rangle=1$, we have $\operatorname{det} L_{n}=1-\lambda^{2}$ (cf. (3.94a) and (3.97)). Thus, the equality (2.11) derived from (2.10) implies that the determinant of $V_{n}$,

$$
\begin{aligned}
\operatorname{det} V_{n} & =\operatorname{det}\left(I+h \frac{\lambda}{1-\lambda^{2}} A_{n} L_{n}\right) \\
& =\operatorname{det}\left(I-\lambda S_{n}+h \lambda A_{n}\right) \operatorname{det}\left(\frac{1}{1-\lambda^{2}} L_{n}\right),
\end{aligned}
$$

is an $n$-independent quantity. Therefore, both the trace and the determinant of $S_{n}-h A_{n}$ must be $n$-independent. The $n$-independence of $\operatorname{tr}\left(S_{n}-h A_{n}\right)$ is already satisfied by setting $a_{n}=a$. The determinant of $S_{n}-h A_{n}$ can be computed as

$$
\begin{aligned}
\operatorname{det}\left(S_{n}-h A_{n}\right) & =\operatorname{det}\left[S_{n}-2 \mathrm{i} h a \widetilde{S}_{n-1}\left(S_{n}+\widetilde{S}_{n-1}\right)^{-1}-2 h b_{n}\left(S_{n}+\widetilde{S}_{n-1}\right)^{-1}\right] \\
& =\frac{\operatorname{det}\left[\left(1-2 h b_{n}\right) I+S_{n} \widetilde{S}_{n-1}-2 \mathrm{i} h a \widetilde{S}_{n-1}\right]}{\operatorname{det}\left(S_{n}+\widetilde{S}_{n-1}\right)} \\
& =\frac{\left(1+\left\langle\boldsymbol{S}_{n}, \widetilde{\boldsymbol{S}}_{n-1}\right\rangle-2 h b_{n}\right)^{2}+1+4(h a)^{2}-\left\langle\boldsymbol{S}_{n}, \widetilde{\boldsymbol{S}}_{n-1}\right\rangle^{2}}{-2\left(1+\left\langle\boldsymbol{S}_{n}, \widetilde{\boldsymbol{S}}_{n-1}\right\rangle\right)}
\end{aligned}
$$


which coincides with its boundary value determined by (3.103). This results in a quadratic equation in $h b_{n}$, i.e.,

$$
\begin{aligned}
2\left(h b_{n}\right)^{2} & -2\left(1+\left\langle\boldsymbol{S}_{n}, \widetilde{\boldsymbol{S}}_{n-1}\right\rangle\right) h b_{n} \\
& +2(h a)^{2}+\left[h b(2-h b)-(h a)^{2}\right]\left(1+\left\langle\boldsymbol{S}_{n}, \widetilde{\boldsymbol{S}}_{n-1}\right\rangle\right)=0 .
\end{aligned}
$$

For sufficiently small $h(0<|h| \ll 1)$, the proper solution of this quadratic equation is given by

$$
h b_{n}=\frac{2(h a)^{2}+\left[h b(2-h b)-(h a)^{2}\right]\left(1+g_{n}\right)}{1+g_{n}+\sqrt{\left(1+g_{n}\right)^{2}-4(h a)^{2}-2\left[h b(2-h b)-(h a)^{2}\right]\left(1+g_{n}\right)}},
$$

where $g_{n}:=\left\langle\boldsymbol{S}_{n}, \widetilde{\boldsymbol{S}}_{n-1}\right\rangle$. Substituting this local expression for $h b_{n}$ into (3.102), we obtain an integrable time discretization of (3.98); this time discretization is essentially equivalent to (B.18) in [15] (see also (26) in [16] and (3.10) in [17]). We write the equation of motion for three important cases: the case $b=0$,

$$
\begin{aligned}
& \widetilde{\boldsymbol{S}}_{n}-\boldsymbol{S}_{n}=\boldsymbol{\Delta}_{n}^{+}\left\{h a \frac{\boldsymbol{S}_{n} \times \widetilde{\boldsymbol{S}}_{n-1}}{1+\left\langle\boldsymbol{S}_{n}, \widetilde{\boldsymbol{S}}_{n-1}\right\rangle}\right. \\
& \left.+\left[1-\sqrt{1-2(h a)^{2} \frac{1-\left\langle\boldsymbol{S}_{n}, \widetilde{\boldsymbol{S}}_{n-1}\right\rangle}{\left(1+\left\langle\boldsymbol{S}_{n}, \widetilde{\boldsymbol{S}}_{n-1}\right\rangle\right)^{2}}}\right] \frac{\boldsymbol{S}_{n}+\widetilde{\boldsymbol{S}}_{n-1}}{2}\right\}, \quad\left\langle\boldsymbol{S}_{n}, \boldsymbol{S}_{n}\right\rangle=1
\end{aligned}
$$

the case $h b(2-h b)=(h a)^{2}$,

$$
\begin{aligned}
\widetilde{\boldsymbol{S}}_{n}-\boldsymbol{S}_{n} & =\boldsymbol{\Delta}_{n}^{+}\left\{h a \frac{\boldsymbol{S}_{n} \times \widetilde{\boldsymbol{S}}_{n-1}}{1+\left\langle\boldsymbol{S}_{n}, \widetilde{\boldsymbol{S}}_{n-1}\right\rangle}\right. \\
& \left.+\left[1-\sqrt{1-\frac{4(h a)^{2}}{\left(1+\left\langle\boldsymbol{S}_{n}, \widetilde{\boldsymbol{S}}_{n-1}\right\rangle\right)^{2}}}\right] \frac{\boldsymbol{S}_{n}+\widetilde{\boldsymbol{S}}_{n-1}}{2}\right\}, \quad\left\langle\boldsymbol{S}_{n}, \boldsymbol{S}_{n}\right\rangle=1 ;
\end{aligned}
$$

and the case $a=0$,

$$
\begin{aligned}
& \frac{1}{\delta}\left(\widetilde{\boldsymbol{S}}_{n}-\boldsymbol{S}_{n}\right) \\
&= \boldsymbol{\Delta}_{n}^{+}\left[\frac{\boldsymbol{S}_{n}+\widetilde{\boldsymbol{S}}_{n-1}}{1+\left\langle\boldsymbol{S}_{n}, \widetilde{\boldsymbol{S}}_{n-1}\right\rangle+\sqrt{\left(1+\left\langle\boldsymbol{S}_{n}, \widetilde{\boldsymbol{S}}_{n-1}\right\rangle\right)^{2}-2 \delta\left(1+\left\langle\boldsymbol{S}_{n}, \widetilde{\boldsymbol{S}}_{n-1}\right\rangle\right)}}\right], \\
& \delta:=h b(2-h b), \quad\left\langle\boldsymbol{S}_{n}, \boldsymbol{S}_{n}\right\rangle=1 .
\end{aligned}
$$


The first and second cases provide time discretizations of the lattice Heisenberg ferromagnet model $[(3.98)$ with $b=0]$, while the third case gives a discrete-time analogue of its simplest higher symmetry [(3.98) with $a=0$ ]. Note that the first case $b=0$ can be identified with (6.8) in [15]. All these difference schemes are seemingly highly implicit, that is, the value $\widetilde{\boldsymbol{S}}_{n}$ is not written explicitly in a closed form in terms of $\boldsymbol{S}_{n}, \boldsymbol{S}_{n+1}$, and $\widetilde{\boldsymbol{S}}_{n-1}$, but this is not a serious drawback. Indeed, we can obtain the power series expansion of $\widetilde{\boldsymbol{S}}_{n}$ in $h$ (or $\delta$ ) to any order successively. Moreover, we can compute the exact value of $\widetilde{\boldsymbol{S}}_{n}$ by the following steps. First, we take the scalar product between the equation of motion and $\boldsymbol{S}_{n+1}$ to obtain an equation for $\left\langle\boldsymbol{S}_{n+1}, \widetilde{\boldsymbol{S}}_{n}\right\rangle$, wherein $\widetilde{\boldsymbol{S}}_{n}$ appears only through the form $\left\langle\boldsymbol{S}_{n+1}, \widetilde{\boldsymbol{S}}_{n}\right\rangle$. Then, the proper solution of this equation can be found in an $O(h)$ (or $O(\delta)$ ) neighborhood of $\left\langle\boldsymbol{S}_{n+1}, \boldsymbol{S}_{n}\right\rangle$. Substituting it back into the original equation of motion, we arrive at a linear equation for $\widetilde{\boldsymbol{S}}_{n}$ that can be solved straightforwardly.

In this subsection, we have obtained the time discretizations of the first two flows of the lattice Heisenberg ferromagnet hierarchy in a unified way. Our derivation is original and easy to follow, but not fully systematic, as some parts are based on heuristic treatments. It would be interesting to investigate whether these time discretizations can be derived in a systematic manner from the time discretizations of the Ablowitz-Ladik lattice obtained in subsection 3.2, using Ishimori's approach [51].

\section{Concluding remarks}

In this paper, we have developed an effective method for obtaining proper time discretizations of integrable lattice systems in $1+1$ dimensions. This method, which is based on the zero-curvature condition (2.6), allows us to obtain local equations of motion that can determine the time evolution uniquely. Using this method, we constructed new time discretizations of the Toda lattice, the Ablowitz-Ladik lattice, the Volterra lattice, and the modified Volterra lattice, while we obtained the same time discretizations of the lattice Heisenberg ferromagnet model and its symmetry as those in [15-17]. It should be stressed that this is a systematic method and also applies to other integrable lattice systems. As a bonus, we were able to derive ultradiscrete analogues of the Volterra lattice and the modified Volterra lattice involving one arbitrary parameter $L$, namely, (3.77) and (3.93). Each of these ultradiscrete equations, as well as its time reversal, appears to form a hierarchy of mutually commuting flows labelled by the parameter $L$. Note that the ultradiscrete equations such as (3.77) and (3.93) allow straightforward "multicomponent" generalizations. Indeed, for example, if we express the de- 
pendent variable and the parameter in the form $U_{n}=U_{n}^{(1)}+U_{n}^{(2)} \boldsymbol{i}, U_{n}^{(j)} \in \mathbb{Z}$ and $L=L^{(1)}+L^{(2)} \boldsymbol{i}>0, L^{(j)} \in \mathbb{Z}$, where $\boldsymbol{i}$ is an irrational number, and substitute them into (3.77), we can uniquely determine the time evolution of the "two-component" system starting from a given initial condition. It would be very interesting to investigate the special case wherein the irrational $\boldsymbol{i}$ is arbitrarily close to 1 .

A notable feature of our method is that the time discretization does not modify the integrable hierarchy to which the original lattice system belongs. Thus, the integrals of motion and the functional form of the solutions remain invariant; only the time dependence of certain parameters corresponding to the angle variables in the solutions is changed (cf. [2, 3, 18]). Such a time discretization can be identified with the spatial part of an auto-Bäcklund transformation of the continuous-time lattice system or, from a more unified point of view, an auto-Bäcklund transformation of the whole hierarchy of commuting flows. Therefore, any time discretization obtained by our method preserves the qualitative properties of the original integrable hierarchy and thus is expected to serve as an excellent scheme for numerical integration of the continuous-time lattice system. This is in contrast with other known methods that generally modify the integrable hierarchy to find a time discretization in the local form. As is illustrated in subsection 3.2, our method can also provide "almost integrable" numerical schemes that far surpass the Ablowitz-Ladik-Taha local schemes [2, 3, 5, 41] in approximation accuracy.

In our approach, a local-in-space time discretization is always derived from a global-in-space time discretization involving "nonlocal" auxiliary variables. The obtained local equations of motion generally determine both the forward and backward time evolution uniquely under the specified boundary conditions; thus, they indeed give a discrete-time analogue of evolutionary differential-difference equations. One might consider that the original nonlocal time discretization no longer has any use once the local discretization has been derived from it; however, this is often not the case. In actually solving an initial-value problem, the nonlocal time discretizations often provide crucial information on the attributes of the solution, such as the real-valuedness, positivity, and rationality with respect to the initial data and the parameters; it appears that the local discretizations are not useful for establishing such properties directly. Let us illustrate this point with one instructive example, namely, the discrete-time Ablowitz-Ladik lattice in the case $a=b=0$ studied in subsection 3.2. The global scheme (3.28) (cf. (3.27)) is free from irrational functions; consequently, under the decaying boundary conditions (cf. (3.24) ), the discrete-time updates of the dependent variables are given by a rational mapping. On the other hand, the local scheme (3.34) with 
(3.32) involves a square root; thus, it is extremely difficult to foresee that the time evolution can be described by a rational mapping. However, these two schemes naturally define the same time evolution. Therefore, the quantity inside the square root in (3.32) is always equal to the square of a rational function of the dependent variables at the previous moment and the parameters; typically, one encounters the form

$$
\sqrt{(1-\hat{c} \hat{d})^{2}\left(1+f_{n}\right)^{2}}
$$

where $\hat{c}$ and $\hat{d}$ depend on the "step size" parameter $h$ through (3.30). The rational function $f_{n}$ involves the dependent variables and the parameters appearing in (3.34), and is of order $O(h)$. In addition, it vanishes when the dependent variables are identically zero. For sufficiently small $|h|(\ll 1)$, this square root is extracted as $(1-\hat{c} \hat{d})\left(1+f_{n}\right)$. However, for relatively large $|h|$, this is not obvious; even when $\hat{c} \hat{d}<1$, the values of the dependent variables have to satisfy rather restrictive conditions. One simple way to bypass this sign problem is to consider the square root as being defined by its Taylor expansion for small $|h|$ or small amplitudes of the dependent variables and then to extend the domain of the definition by analytic continuation; this is briefly explained in subsection 3.2. Thus, this example also illustrates the difficulty of the sign problem in computing updates of the dependent variables using the local equations of motion.

In this paper, we mainly considered the "constant" boundary conditions at spatial infinity (cf. (2.9)) and assumed simple boundary values of the dependent variables. Note, however, that our method is not sensitive to the boundary values of the dependent variables and is also applicable to other boundary conditions, including periodic, nonvanishing, or nonconstant boundary conditions. Indeed, as is clear from the description in subsection 2.3, one can freely modify the boundary conditions for $V_{n}$ as long as they determine a definite value for the right-hand side of (2.14) or (2.15) that is $n$-independent. Alternatively, one can first set each value of (2.14) or (2.15) as a "constant" free parameter and then elicit the corresponding boundary conditions for the dependent variables. In either case, one should take care to identify the proper solution of the resulting algebraic system (cf. subsection 2.4). We can also understand in a more intuitive way that the time discretizations derived in this paper are integrable under other suitable boundary conditions; note that the conservation laws should be derived from the local equations of motion using only local operations, i.e., without referring to the boundary conditions.

Very recently, Adler, Bobenko, and Suris [36,37] successfully classified discrete integrable systems on quad-graphs using the notion of three-dimensional 
consistency [35]. In particular, under some assumptions, they presented a short but complete list of one-field integrable equations defined by polynomial relations of degree one in each of the four arguments. Their results have been attracting a lot of interest from researchers; for example, click on the NASA ADS link at

$$
\text { http://arxiv.org/abs/nlin/0202024. }
$$

The time discretizations of polynomial lattice systems obtained by our method generally contain irrational nonlinearity; the nonlinear terms are determined through the proper solution of an "ultralocal" algebraic equation of degree higher than one. Thus, such time discretizations essentially lie outside the class considered by Adler et al. [36, 37], although their three-dimensional consistency can, in principle, be investigated using the approach outlined in the last paragraph of subsection 2.5. This probably partially explains why their list of one-field integrable equations is short.

\section{Acknowledgments}

The author thanks Professor J. Matsukidaira, Professor K. Nishinari, Professor S. Tsujimoto, Professor K. Toda, Professor T. Takenawa, Dr. F. Sugino, Dr. Z. Tsuboi, and Dr. K. Maruno for their useful comments and discussions.

\section{References}

[1] Y. B. Suris: The Problem of Integrable Discretization: Hamiltonian Approach (Birkhäuser, Basel, 2003).

[2] M. J. Ablowitz and J. F. Ladik: Stud. Appl. Math. 55 (1976) 213.

[3] M. J. Ablowitz and J. F. Ladik: Stud. Appl. Math. 57 (1977) 1.

[4] M. J. Ablowitz and J. F. Ladik: J. Math. Phys. 17 (1976) 1011.

[5] T. R. Taha and M. J. Ablowitz: J. Comp. Phys. 55 (1984) 192.

[6] Y. B. Suris: Phys. Lett. A 234 (1997) 91.

[7] Y. B. Suris: Inv. Probl. 13 (1997) 1121.

[8] R. Hirota: The Direct Method in Soliton Theory (Cambridge Univ. Press, Cambridge, 2004) edited and translated by A. Nagai, J. Nimmo and C. Gilson. 
[9] H. Flaschka: Phys. Rev. B 9 (1974) 1924.

[10] H. Flaschka: Prog. Theor. Phys. 51 (1974) 703.

[11] S. V. Manakov: Sov. Phys. JETP 40 (1975) 269.

[12] D. Takahashi and J. Matsukidaira: Phys. Lett. A 209 (1995) 184.

[13] T. Tokihiro, D. Takahashi, J. Matsukidaira and J. Satsuma: Phys. Rev. Lett. 76 (1996) 3247.

[14] F. W. Nijhoff, G. R. W. Quispel and H. W. Capel: Phys. Lett. A 95 (1983) 273.

[15] G. R. W. Quispel, F. W. Nijhoff, H. W. Capel and J. van der Linden: Physica A 125 (1984) 344.

[16] F. W. Nijhoff, H. W. Capel, G. L. Wiersma and G. R. W. Quispel: Phys. Lett. A 103 (1984) 293.

[17] F. Nijhoff and H. Capel: Acta Appl. Math. 39 (1995) 133.

[18] F. Kako and N. Mugibayashi: Prog. Theor. Phys. 61 (1979) 776.

[19] Y. B. Suris: Inv. Probl. 16 (2000) 1071.

[20] S. J. Orfanidis: Phys. Rev. D 18 (1978) 3822.

[21] M. J. Ablowitz: Stud. Appl. Math. 58 (1978) 17.

[22] K. Kajiwara and A. Mukaihira: J. Phys. A: Math. Gen. 38 (2005) 6363.

[23] S. J. Orfanidis: Phys. Rev. D 18 (1978) 3828.

[24] E. Date, M. Jimbo and T. Miwa: J. Phys. Soc. Jpn. 51 (1982) 4116.

[25] T. Sadakane: J. Phys. A: Math. Gen. 36 (2003) 87.

[26] G. L. Lamb, Jr.: Rev. Mod. Phys. 43 (1971) 99.

[27] A. C. Scott, F. Y. F. Chu and D. W. McLaughlin: Proc. IEEE 61 (1973) 1443.

[28] R. M. Miura (editor): Bäcklund Transformations, the Inverse Scattering Method, Solitons, and Their Applications (Lect. Notes in Math. 515, Springer, Berlin, 1976). 
[29] B. G. Konopelchenko: Phys. Lett. A 87 (1982) 445.

[30] H. D. Wahlquist and F. B. Estabrook: Phys. Rev. Lett. 31 (1973) 1386.

[31] V. Papageorgiou, B. Grammaticos and A. Ramani: Phys. Lett. A 179 (1993) 111.

[32] V. P. Spiridonov, S. Tsujimoto and A. S. Zhedanov: Commun. Math. Phys. 272 (2007) 139.

[33] K. Kajiwara and Y. Ohta: J. Phys. Soc. Jpn. 77 (2008) 054004.

[34] R. Hirota: J. Phys. Soc. Jpn. 43 (1977) 2079.

[35] F. W. Nijhoff and A. J. Walker: Glasgow Math. J. 43A (2001) 109.

[36] V. E. Adler, A. I. Bobenko and Y. B. Suris: Commun. Math. Phys. 233 (2003) 513.

[37] V. E. Adler, A. I. Bobenko and Y. B. Suris: Funct. Anal. Appl. 43 (2009) 3.

[38] R. K. Dodd: J. Phys. A: Math. Gen. 11 (1978) 81.

[39] Y. B. Suris: Phys. Lett. A 206 (1995) 153.

[40] F. Pempinelli, M. Boiti and J. Leon: Proceedings of the First Workshop on Nonlinear Physics, Theory and Experiment (Gallipoli, Italy, 1995) edited by E. Alfinito et al. (World Scientific, Singapore, 1996) pp. 261.

[41] T. R. Taha and M. J. Ablowitz: J. Comp. Phys. 55 (1984) 203.

[42] L. D. Faddeev and L. A. Takhtajan: Hamiltonian Methods in the Theory of Solitons (Springer, Berlin, 1987) translated by A. G. Reyman.

[43] J. Matsukidaira, J. Satsuma, D. Takahashi, T. Tokihiro and M. Torii: Phys. Lett. A 225 (1997) 287.

[44] D. Takahashi and J. Matsukidaira: J. Phys. A: Math. Gen. 30 (1997) L733.

[45] D. Takahashi and J. Satsuma: J. Phys. Soc. Jpn. 59 (1990) 3514.

[46] R. Hirota: J. Phys. Soc. Jpn. 35 (1973) 289.

[47] M. Wadati: Prog. Theor. Phys. Suppl. 59 (1976) 36. 
[48] M. J. Ablowitz and J. F. Ladik: J. Math. Phys. 16 (1975) 598.

[49] R. Hirota and S. Tsujimoto: J. Phys. Soc. Jpn. 64 (1995) 3125.

[50] E. K. Sklyanin: Funct. Anal. Appl. 16 (1982) 263.

[51] Y. Ishimori: J. Phys. Soc. Jpn. 51 (1982) 3417.

[52] F. D. M. Haldane: J. Phys. C: Solid State Phys. 15 (1982) L1309.

[53] L. Faddeev: Recent Advances in Field Theory and Statistical Mechanics (Les Houches Summer School, Session XXXIX, 1982) edited by J.-B. Zuber and R. Stora (North-Holland, Amsterdam, 1984) pp. 561.

[54] V. S. Gerdjikov, M. I. Ivanov and Y. S. Vaklev: Inv. Probl. 2 (1986) 413. 